\title{
State-space Manifold and Rotating Black Holes
}

\author{
Stefano Bellucci ${ }^{a}{ }^{*}$ Bhupendra Nath Tiwari ${ }^{a \dagger}$ \\ ${ }^{a}$ INFN-Laboratori Nazionali di Frascati \\ Via E. Fermi 40, 00044 Frascati, Italy.
}

\begin{abstract}
We study a class of fluctuating higher dimensional black hole configurations obtained in string theory/ $M$-theory compactifications. We explore the intrinsic Riemannian geometric nature of Gaussian fluctuations arising from the Hessian of the coarse graining entropy, defined over an ensemble of brane microstates. It has been shown that the state-space geometry spanned by the set of invariant parameters is non-degenerate, regular and has a negative scalar curvature for the rotating Myers-Perry black holes, Kaluza-Klein black holes, supersymmetric $A d S_{5}$ black holes, $D_{1}-D_{5}$ configurations and the associated BMPV black holes. Interestingly, these solutions demonstrate that the principal components of the state-space metric tensor admit a positive definite form, while the off diagonal components do not. Furthermore, the ratio of diagonal components weakens relatively faster than the off diagonal components, and thus they swiftly come into an equilibrium statistical configuration. Novel aspects of the scaling property suggest that the brane-brane statistical pair correlation functions divulge an asymmetric nature, in comparison with the others. This approach indicates that all above configurations are effectively attractive and stable, on an arbitrary hypersurface of the state-space manifolds. It is nevertheless noticed that there exists an intriguing relationship between non-ideal inter-brane statistical interactions and phase transitions. The ramifications thus described are consistent with the existing picture of the microscopic CFTs. We conclude with an extended discussion of the implications of this work for the physics of black holes in string theory.
\end{abstract}

Keywords: Rotating Black Holes; State-space Geometry; Statistical Configurations, String Theory, $M$-Theory.

PACS numbers: 04.70.-s Physics of black holes; 04.70.Bw Classical black holes; 04.70.Dy Quantum aspects of black holes, evaporation, thermodynamics; 04.50. Gh Higher-dimensional black holes, black strings, and related objects.

*bellucci@lnf.infn.it

†tiwari@lnf.infn.it 


\section{Contents:}

1. Introduction.

2. State-space Geometry.

3. Rotating Black Holes:

3.1 Myers-Perry Black Holes.

3.2 Kaluza-Klein Black Holes.

3.3 Supersymmetric $A d S_{5}$ Black Holes.

$3.4 \quad D_{1}-D_{5}$ Configurations.

3.5 BMPV Black Holes.

4. Conclusion and Discussion.

\section{Introduction}

Motivated from the physics and mathematics of higher dimensional brane configurations and their microscopic descriptions, we focus on the possible investigations of covariant intrinsic thermodynamic Riemannian geometries for a class of rotating black hole solutions obtained in either string theory or $M$-theory compactifications. Our study is prompted in part by the recent insight perceived from the string theory solutions, which may in turn impel significant progress towards an understanding of the nature of microscopic data, in the limiting case of extremal and near-extremal black brane configurations. In particular, it is well-known that one can count the microstates for certain rotating and non-rotating black brane configurations carrying the same charges, angular momenta and energy, with a microscopic entropy $S_{\text {micro }}$ like that of the corresponding macroscopic configurations having Bekenstein-Hawking entropy $S_{B H}=\frac{A}{4 G}$, see for instance [1] 3$]$.

To refine this issue, one should first note that the concept of AdS/CFT correspondence 4 7 suggests that the $S_{\text {micro }}$ counts the microstates of the black brane configuration that belong to the boundary CFT, which is dual to the gravitational description. This has been very useful in understanding the black brane entropy, which is based on the coarse graining of an ensemble of brane microstates parameterized by a finite number of asymptotic parameters. We believe that the underlying space-time geometries may be capturing generic CFT states of the black holes and black branes with non-zero microscopic degeneracy, and thus capturing enough of them to account for their fluctuating horizon area. Here, we will use this result as a crucial ingredient in the realization of possible state-space fluctuations around an equilibrium statistical configuration of the higher dimensional rotating black hole solutions.

The fact that we are only looking at a special class of Gaussian fluctuations means that we are necessarily restricting the degrees of freedom of the interested configurations. Thus, the crucial idea would be first to develop an intrinsic Riemannian geometric notion, for synthesizing the statistical correlations among the underlying microstates of the various rotating black hole solutions. To fully support this construction, we shall point out an adequate ground for the microscopic perspective ratifying the state-space geometry. The metric tensor arises from the negative Hessian matrix of the corresponding coarse graining entropy defined over a large number of brane microstates characterizing the chosen black hole macroscopic configuration. Here, the important progress is thus to appreciate how the microstate geometries may capture enough entropy to account for statistical correlations and their dependence on finitely many conserved electricmagnetic charges, angular momenta and other possible physical charges, if any.

As in the classical theory of four dimensional black holes, the uniqueness theorems assert that the parameters of a black hole are precisely those which correspond to certain conserved quantities, viz., the mass, angular momenta, and promising conserved charges, if any, associated with local gauge symmetries. Moreover, we know that the possible black hole solutions of the four-dimensional Einstein-Maxwell theory are only the Kerr-Newman black holes 8 10. More strongly, this result precludes the possibility that the black hole possesses higher multipole moments (e.g., a mass quadrupole or a charge dipole) that are not completely fixed by the values of conserved charges. It is however worth to notice that the higher dimensional rotating black branes may in general violate the black hole uniqueness theorems, which exist for the ordinary four dimensional configurations [11,12. Another consequence is that the microscopic states, that are responsible for the large degeneracy implied by the Bekenstein-Hawking entropy of a rotating configuration, are however invisible at the level of the classical gravitational theory, but their radiative effects may be observed in the corresponding state-space geometry.

There is indeed a significant body of evidences that supports the idea of the state-space geometry for the Myers-Perry black holes, which are the simplest rotating black hole configurations in five dimensional space-times and thus capture the basic notion of our geometric investigations. Here, we will focus on the recent discovery that the five-dimensional black holes exhibit qualitatively new properties, which are rather 
not shared by their four-dimensional siblings. We would like to investigate this from the perspective of state-space manifolds associated with spherical horizon topology black holes, where the conventional notions of uniqueness theorems do apply [13. In short, we would investigate on certain possible developments, which may notably be appreciated in the higher dimensional versions of the Kerr solutions constructed by the Myers and Perry [14] and in the other associated brane configurations.

The statistical importance of charged supersymmetric black holes in string theory has originally been motivated by the study of Strominger and Vafa [1. They have shown that the microscopic understanding of black holes in string theory may be characterized by the parameters of the solutions, and in particular, the charges of supersymmetric black holes serve as the tag marks to identify their microscopic constituents in the string theory. Moreover, the phase-space of the supersymmetric black hole solutions turns out to be drastically constrained and is subject to various powerful non-renormalization theorems and state-counting techniques. However, it is known that the neutral black holes in $M$-theory carry a minimal set of quantum numbers, viz., mass and angular momentum [14]. Therefore, it is hard to restrict the phase-space into a sector in which it is simple enough to count the microstates. Besides the microscopic deficiency, we find for these solutions that it is not difficult to give an intrinsic state-space meaning to the associated statistical fluctuations, under the Gaussian approximation.

There exist vacuum black holes in $M$-theory that can be mapped to certain well-defined bound states of $D$-brane configurations in string theory, which in an intriguing limit become asymptotically flat. In particular, we shall restrict our attention on the state-space manifolds of five-dimensional dyonic black hole solutions described in Kaluza-Klein theory [15. It has been shown in Ref. [16] that, in an ascription akin to the decoupling limit in AdS/CFT, such black holes include the five-dimensional asymptotically flat neutral rotating solutions of Myers and Perry [14]. Furthermore, one can view these black holes as the Myers-Perry black holes placed at the tip of the Taub-NUT geometry. Our purpose would here be to study and compare the nature of their state-space interactions, viz., for the Kaluza-Klein black holes and that of the Myers-Perry black holes and thus one may simultaneously analyze whether they both pertain a well-defined state-space.

The case of the Kaluza-Klein configuration implies that an addition of electric charge or energy to the extremality condition leads to a finite horizon black hole at the tip of the cigar solution, which is being fibered with the spherical horizon of the corresponding four-dimensional black hole. The corresponding magnetically charged Kaluza-Klein black holes may thus be viewed as the five-dimensional black holes with $S^{3}$ horizon topology, which may in turn be localized inside the Taub-NUT geometry. On the other hand, it is much more illuminating to note that both descriptions of this model are considerably interesting for our geometric analysis. In particular, it is worth to recall that the electric charges do not correspond to a boost, but to the rotations of the five dimensional black holes, which are aligned along the Kaluza-Klein circle [16. We find that these rotations may be analyzed from the perspective of general co-ordinate transformations on the concerned state-space manifold. This also follows directly from the fact that the component of the rotation that is not aligned with the fiber of the five dimensional solution determines the four-dimensional rotation, which describes the usual four dimensional Kerr-Newman black holes.

Microscopically, we can count the microstates of Kaluza-Klein configurations by taking the tensor product of $T^{6}$ with the $S^{1}$ and view it as a vacuum solution in the $M$-theory, with the Kaluza-Klein circle being the $M$-theory compactification circle. The starting point of our discussion may thus be concerned with the consideration of usual duality relations between the $M$-theory and type-IIA string theory, which comprise certain electric and magnetic charges corresponding to the given number of constituent $D_{0}$ and $D_{6}$-branes [16]. In this case, it turns out that the quantization conditions exist for the individual electric and magnetic charges, which may simply be written in terms of the net number of corresponding $D$-branes. Furthermore, this yields that the net degeneracy of the $D_{0}$ and $D_{6}$-brane microstates may be given by the central charge of the conformal field theory. More precisely, we would like to appraise how our state-space geometry of an extremal Kaluza-Klein black hole entangles with its degeneracy of CFT microstates, and it describes the corresponding $D_{0}-D_{6}$ microscopic configuration.

This is positive and compelling evidence for the identification of black hole entropy formulas, and thus of the derived state-space quantities, which may indeed be done so, just by taking the T-dual configurations in which the microscopic descriptions become more transparent. For simplicity, let us consider the case of non rotating four-dimensional solutions. Then the supersymmetric four-charge black hole configurations in Type II string theory compactified on $T^{6}$ have many possible choices for the charges, which in general are related by U-duality. It has been shown in [17] that one may explain the microscopic origin of the Kaluza-Klein black holes, just by considering four stacks of $D_{3}$-branes. Among these, any two stacks intersect over a line, while the rest four intersect at a point. Moreover, the orientation of the first three stacks can be chosen arbitrarily, but the orientation of the last set of $D_{3}$-branes is required to be fixed, in order to preserve the $\mathcal{N}=2$ supersymmetry. In the case when the number of branes in each stack remains same, then one finds that the torus moduli remain constant. In particular, the corresponding solution reduces to the extremal Reissner-Nordström black hole, which has a non-interacting state-space geometry [18. Here, one educes that the covariant intrinsic structures may easily be determined from the entropy (or mass) which grows 
with the number of $D_{3}$-branes wrapped around the torus.

Investigations of the relationship between the type IIB superstring theory and Yang-Mills theory have further motivated us to analyze the state-space structure of the supersymmetric $A d S_{5}$ black holes. In particular, the spectrum of the microstates in $\frac{1}{2}$-BPS sector has extensively been studied in both the string theory and $\mathcal{N}=4$ Yang-Mills theory [19]20. It has been known that the spectrum of type IIB superstring theory contains a large class of BPS states, when compactified on $A d S_{5} \times S^{5}$. Thus, it would be interesting to describe the nature of possible statistical correlations for these black hole configurations, under the general consideration of microscopic superconformal field theories. In turn, we identify that the nonzero $U(1) \subset S O(6)$ momenta or certain R-charges associated with the possible fractional BPS states serve as the co-ordinates for our state-space geometry of these configurations. More recently, some pioneering works have also been done in the supergravity configurations and in super Yang-Mills theories, which provide an extent of confirmation for our investigations, see for example $21,25$.

It would thus be interesting to consider the state-space geometry in a certain specific supersymmetric sector. In particular, the fact that the $\frac{1}{16}$-BPS states preserve 2 real supersymmetries provides viable support for our geometric examination. Our analysis shows that the underlying microscopic configurations should have a finite and regular correlation volume. One such motivation for the concerned behavior of the supersymmetric configurations comes from the study of the $A d S_{5}$ black holes, which have been rather recently obtained from the five dimensional gauged supergravity theories 26 30. It turns out that these black holes carry three $U(1)^{3} \subset S O(6)$ momenta in the $S^{5}$, as well as two $U(1)^{2} \subset S O(4)$ in the $A d S_{5}$, which define the co-ordinate charts for the underlying state-space geometry. In order to have a regular horizon black hole, it may however be urged that the angular momenta in the $A d S_{5}$ should be nonzero: otherwise the black hole solutions of interest would develop certain naked singularities 31 32. In particular, the presence of angular momenta in $A d S_{5}$ forces them to preserve a symmetry not larger than the $\frac{1}{16}$-supersymmetry. We observe in the present case that the associated state-space turns out to be an intrinsic real Riemannian geometry, which effectively implies an attractive statistical configuration.

To be concrete, we shall be interested here in the $A d S_{5} \times S^{5}$ black hole configurations. In particular, we shall consider five dimensional $\mathcal{N}=1$ gauged supergravity coupled with $n$ abelian vector multiplets, which turns out to be more convenient, in order to deal with these solutions, including certain general supergravity theories of interest. For simplicity, we consider the supergravity black holes in the symmetric spaces, which involve a self-dual angular momentum in $A d S_{5}$ described in 27. Before commencing further details, we wish to recall that the physical quantities which define our state-space geometry are only the $U(1)^{n}$ charges $Q_{i}$ and the self-dual angular momentum $J=\frac{J_{1}+J_{2}}{2}$, where the $J_{1}, J_{2}$ are the Cartan generators of $S O(4)$ rotations of the $A d S_{5}$. Note, further, that the Bekenstein-Hawking entropy of such black holes may easily be given by the horizon area of a squashed 3-sphere. Our computation thus allows for compact expressions for the state-space quantities. We also notice that there exists a set of interesting scaling properties for these underlying configurations.

Another consequence is that there are $n+1$ independent charges carried by these black holes, viz., $n$ electric charges $Q_{i}$ 's and a self-dual angular momentum $J$, but there are only $n$ independent physical parameters $q_{i}$ for the genuine solutions. This is due to the fact that there exists an intriguing relationship between the charges, In particular, if one tries to express the macroscopic entropy in terms of the charges $Q_{i}$ and the rotation $J$, then there exists an ambiguity in its expression, see for more details [33, 34]. Thus, one may take advantage of this ambiguity and write the macroscopic entropy in terms of the electric charges accompanied by these black hole configurations. For a symmetric space, Kim et. al. found that there exists an appropriate combination: $C^{i j k} \bar{X}_{i} Q_{j} Q_{k}$ of the charges $Q_{i}$ and vacuum scalars $\bar{X}_{i}$, which defines an illuminating formula for the concerned entropy, $S_{B H}=2 \pi \sqrt{D^{i j} Q_{j} Q_{k}-4 c J}$, see for the details [35. Here, the constant $c$ actually defines the central charge of a 4 dimensional holographic superconformal field theory living on the boundary, and may as well be computed from the dual gravity data. In particular, for the choice of $\mathcal{N}=4 S U(N)$ Yang-Mills theory, the central charge $c$ may be normalized to be $c=N^{2} / 4$. Our analysis thus determines the allowed physical domain for the statistical correlations and possible invariants of underlying state-space geometry. We find, specifically, that the $A d S_{5}$ black holes are stable under the Gaussian fluctuations, if at least one of the parameter remains fixed.

Notice that the mentioned premises may easily be analyzed from the microscopic duality transformations on the charges, which in the large charge limit imply the existence of a correspondence with the general coordinate transformations on the associated state-space manifold. In fact, the state-space geometry of such a general black brane configuration may be achieved from the parameters of an effective space-time geometry, describing an ensemble of equilibrium microstates. At this juncture, we may demonstrate that the state-space geometry, thus characterized in terms of physical parameters of the black brane configurations, may always be defined as an intrinsic real Riemannian manifold. Up to a certain quotient, corresponding to the flat directions, we nevertheless encounter that most of the known higher dimensional solutions display certain regular, negatively curved state-space manifolds. Accordingly, it turns out, in the present prescription, that the scalar curvature signifies nothing more than the possible attractions, in the underlying statistical 
configurations.

Furthermore, we wish to focus our attention on the statistical pair correlation functions. In particular, we would like to analyze the consequences emerging from the existing macroscopic considerations and intend to indicate the nature of the microscopic configuration. Typically, the approach to our intrinsic geometric study of the black hole thermodynamic starts with the Gaussian fluctuations of the black hole entropy. In particular, the components of the intrinsic Riemannian geometry, thus invoked, may be obtained as the negative Hessian matrix of its macroscopic entropy, computed from the Bekenstein-Hawking-Wald area law. Thus, one may easily analyze possible geometric structures arising from the Gaussian fluctuations and there by try to understand underlying statistical configurations of the higher dimensional black holes.

This article has consequently been organized into the following sections and their respective subsections. The very first section has in turn illuminated several intriguing motivations to examine the statistical fluctuations and thereby initiates why to study our state-space geometry. In section 2, we shall briefly explain what is the state-space geometry based on a large number of equilibrium parameters, characterizing the black hole solution. In section 3, we investigate the state-space geometry for the neutral Myers-Perry black holes, and anticipate two and three charged rotating configurations, in higher dimensional space-times. Section 4 consists of some concluding notes and a set of remarks arising from the state-space geometry. The general implications thus obtained may be divulged, for various possible black holes in string theory and $M$-theory.

\section{Motivations from microstates counting}

In order to see the key points of our state-space investigations, it is worth to note that Mathur has proposed a class of fuzzball solutions by analyzing certain implications of the AdS/CFT correspondence [11 12 36 37]. In particular, Mathur has shown, for the physics of a $D_{1}-D_{5}$-system, that each CFT vacuum of this configuration is dual to the smooth bulk solution, which neither has a horizon nor the loss of information 37. Thus, we may take account of the fuzzball geometry into our state-space quantities, and our geometric observations thus made are based on the well-known fact that the $D_{1}-D_{5}$-configuration acquires a large microscopic degeneracy. The success of this endeavor has in turn led us to a realization that one may similarly procure an appropriate state-space notion for the $D_{1}-D_{5}-P$ fuzzball solutions, which receive correction form the corresponding Kaluza-Klein-momentum [3]. Moreover, this comprehension leads one to accept through the AdS/CFT correspondence that the $D_{1}-D_{5}-P$ black holes may be thought of as an ensemble of fuzzball geometry 36].

Thus, one may clearly see how our intrinsic geometric results open a new fascinating window into the understanding of statistical correlation functions for the black holes in string theory and $M$-theory. The main line of hypothesis has already provided a parallel motivation for much of the striking task on black brane state-space manifolds 38. In particular, many other related investigations including the proposition of Mathur's fuzzballs 37] can be also introduced for the fundamental state-space structures of a class of higher dimensional black brane configurations. To be more specific, we notice an interesting agreement with the Mathur's proposal that there should exist some U-duality frame, in which the non-singular, horizonfree supergravity black hole microstates admit a coarse grained space-time geometric description for these solutions.

In a similar spirit, the intrinsic Riemannian geometry characterizes possible statistical correlations and shows that the underlying $D_{1}-D_{5}$ or $D_{1}-D_{5}-P$ black holes entail an attractive regular state-space configuration. In order to resolve the black hole information paradox, the Mathur's programme has inspired a search for gravitating microstates which are horizon free and non-singular, with the same brane charges as that of the considered black hole [3, 37]. Various computations in string theory have suggested that the usual picture of a black hole arising with an event horizon could be an emergent phenomenon of the empty space with a central singularity, when we coarse grain the configuration over an ensemble of microstates.

Recently, Lunin and Mathur have made a stronger conjecture [39] which connotes that the black hole microstates may be characterized by string theory backgrounds with no horizons. These solutions saturate an exact bound on their angular momentum and have the same set of asymptotic charges, as that of the respective black hole, whose horizon area classically vanishes. Some finite temperature (non-supersymmetric) generalizations appear as well, which correspond to definite black holes with finite size horizons; we may thus be interested in finding state-spaces for the BPS black holes which are already known to have a finite size horizon at zero temperature 11,12. In five space-time dimensions, the complete class of two charge supersymmetric solutions is in fact well-known since the invention of [36]. Thus, it would be inspiring to associate to them the corresponding state-space manifold. Since the number of parameters for such configurations is the same as for the macroscopic supergravity solutions, this might indicate the behaviour of the underlying CFT configurations.

In the case of two charge configurations, the microscopic solutions do appear surprisingly like a blackhole 37. The higher-derivative corrections cause the heterotic string solutions to have well defined two charge small black hole configurations. Both of them have well defined state-space correlations [38. This is 
because they exhibit throat regions, which close off at the compactification radius. The latter may be fixed in terms of the charges of the theory, by employing an associated attractor mechanism. Now, if one computes the Bekenstein-Hawking entropy associated with this radius of capped throat space-time geometry, then one may easily define the contraventions associated with state-space correlations and thus examine them in contrast to the existing microscopic configurations. In particular for the two charge rotating small black branes, it has been demonstrated in 38] that these notions indeed agree with those anticipated in terms of the parameters of underlying statistical configurations.

Supersymmetric five dimensional black holes are of considerable interest in string theory, because they admit simple microscopic descriptions in terms of the charges of underlying $D$-branes [1]. Here, the largest known family of such black holes is the family of four-parameter BMPV solutions [40]. The present work shows that these configurations have an interesting state-space structure, in general. In particular, we discover that the statistical pair correlations satisfy a definite set of scaling properties, with respect to each other, and the underlying statistical systems are effectively an attractive configuration under the Gaussian fluctuations. Clearly the challenge is now to obtain a microscopic description of their entropy, that correctly accounts for the statistical fluctuations into these solutions. What appears to be an obstacle may actually be a very useful ingredient, towards a more complete understanding of the $D_{1}-D_{5}-P$ system.

In this direction, a remarkable aspect of the supergravity solutions is the way in which they capture highly nontrivial constraints between the parameters that arise in their possible microscopic descriptions. As for the BMPV black holes, it has already been known that the condition that the closed casual curves be absent yields appropriate upper bounds on the angular momentum. Such bounds are also supported from the microscopic CFT or worldvolume theory. Similar constraints do arise from our state-space construction, that the supergravity solutions may alternatively be viewed from the requirement that they should admit a thermal deformation. It would indeed be very surprising if string theory could not account for these solutions, which in their low energy supergravity limit seem completely pathology-free and describe an absolute picture for our state-space geometry.

From the viewpoint of thermodynamics, we realize as well that the solutions, which are characterized by the same asymptotic charges as that of the black holes, may be regarded as being only locally stable and correspond to an attractive configuration. However, if we partially maximize the entropy by varying one or more parameters, then we discover the existence of a unique, globally stable, thermodynamic configuration. It is worth to note, in general, that the state-space constraints thus derived, by requiring hyper-planar stabilities, do not merely fix the angular momentum for the given charges, but instead they impose an upper bound on it. This is indeed in an accordance with certain supergravity constraints, which actually require the absence of causal anomalies and similarly impose an upper bound on the allowed angular momentum [4]. For the purpose of future analysis, we thus note that the $D_{1}-D_{5}-P$ black holes of interest may be obtained from the solution of eleven-dimensional supergravity, which may easily be Kaluza-Klein reduced to a solution of type IIA supergravity and then dualized to the type IIB solution, with net $D_{1}, D_{5}$ charges and Kaluza-Klein momentum.

We here study the state-space properties of these black brane solutions and specifically analyze them for the limiting case of spherical horizon configurations. In particular, it has been realized, during the study of stationary, asymptotically flat black hole solutions, that the $D=5$ vacuum Einstein equations admit BMPV black holes, with an event horizon of $S^{3}$ topology which exclusively describes intriguing higher dimensional rotating black holes 13 . It turns out that our state-space geometry determining the nature of statistical configuration only involves the asymptotic charges of underlying black hole solutions. This lies on the fact that these black holes may uniquely be characterized by their asymptotic parameters, viz., conserved charges, mass and angular momentum. Moreover, these charges do not distinguish the black holes of spherical topology from other possible black brane configurations. See for example how the solutions constructed in 42, 43, describe the case of charged black rings. Furthermore, it may be interesting to determine the possible mutual relevance of the state-space quantities, among the various horizon changing solutions.

Nevertheless, one could equivalently work by constructing the associated CFT microstates, whose degeneracy formula gives rise to the microscopic entropy. Thus the statistical pair correlation functions and correlation volume may also be observed for the underlying phase-space configurations. Therefore, it seems feasible, via an appropriate AdS/CFT-map, that one may even predict certain equivalences among the parameters describing the CFT correlators and the co-ordinates of state-space manifolds, for the specific black brane configurations. It would indeed be interesting to ponder, whether this kind of approach can be pushed further, for example to examine certain properties of the correlations in the above possible descriptions, for the various new black branes in $D>4$ space-time solutions. In general, we contemplate that such predictions are not of much help in realizing the statistical pair correlation functions and correlation volume of an arbitrary black brane configuration characterized by a large number of charges, angular momenta, ADM mass and possibly other local charges. In particular, one may easily perceive the order of the difficulty, in the analysis of the concerned statistical configuration, just by knowing the dimension of the state-space manifold. 
However, we notice that there exist certain special configurations, where some amount of supersymmetry is preserved, which turn out to be more tractable than the general configurations.

Furthermore, it may be observed that the string theory/ $M$-theory descriptions play a central role in understanding certain state-space properties of the rotating black holes, or in general black brane configurations, see for instance 1, 13,44,48. In particular, our analysis might deploy the AdS/CFT correspondence, involving the equality of the partition function of a string theory (or $M$-theory) compactified on a space $K$, with asymptotics of form $A d S_{n} \times K$, and that of the associated holographic dual conformal field theory, described on the conformal boundary of the $A d S_{n}$, see [49 54], for some admissible compact spaces and Calabi-Yau compactifications. Moreover, a conjecture exists for type IIA extremal p-dimensional black brane attractors, i.e. that they correspond to $A d s_{p+2} \times S^{8-2 n-p}$ horizon geometries 55 .

Having thus provided a brief account of the higher dimensional black hole configurations, we find interesting to note that there exists an asymptotic expansion of the exact spectrum of the degeneracy formula, for large charges and angular momenta, which not only reproduces the entropy of corresponding black branes in the leading order, but also to the first few subleading orders, which may be given as an expansion in the inverse power of the charges [50,56], and particularly see [57] for the spectrum of half-BPS states in $\mathcal{N}=4$ supersymmetric string theory. Given this correspondence between the microscopic spectrum and the macroscopic entropy of a class of black brane configurations, a natural question would be to assimilate a microscopic CFT origin of the state-space geometric interactions, arising from the degeneracy formula and, in specific, what does this correspondence mean for rotating black holes? However, we find this question beyond the scope of the present interest and would relegate it for the future analysis.

Here, we focus our attention on the asymptotic parameters defining these black hole solutions, which divulge certain associated macroscopic and microscopic acquisitions as well. There by one may critically examine the physical implications arising from the Gaussian fluctuations of the entropy, defining the intrinsic state-space geometry. The present paper thus concentrates on the macroscopic-microscopic descriptions of consistent rotating black brane solutions and discuss the underlying state-space geometry, thus anticipated as certain bound states of the possible configurations arising, either from the $D$-branes, or dual $M$-branes. Therefore, our study of the equilibrium state-space geometry, characterized by certain electric-magnetic charges, other invariant quantities, such as ADM mass, and angular momenta, is well suited for an intriguing application of the AdS/CFT correspondence and vacuum phase-transitions, if any, in the considered black brane configurations.

Moreover, the state-space geometry has been one of the pivotal intervention in the study of thermodynamic structures of a large class of condensed matter systems, black holes or higher dimensional rotating black branes, see for instance [58 71]. In particular, the article 38 describes the nature of the state-space configuration for various extremal and non-extremal black branes, including the multi-centered branes, fractional branes, fuzzy rings and $M$-theory bubbling solutions. It may be observed that our state-space geometry analyzes the most probable interactions between the microstates of the black branes. Specifically, it determines the nature of statistical pair correlation functions, correlation volume and the possible stability criteria under the Gaussian fluctuations, in the underlying equilibrium configurations. Our analysis thus provides an appropriate ground for the implications arising from the physics of black holes in string theory/ $M$-theory.

The applications thus considered are analogous to those mimicked in 38, which give an appropriate notion of statistical interactions, for the bubbling $A d S$ geometry and other well-known configurations. Here, we shall explicitly construct the general features of the state-space configurations, which come from the underlying black brane entropy. It may thus naively be possible to compare the concerned geometric quantities, with the fundamental characteristics of the underlying microscopic configurations. In particular, the state-space, thus constructed, primarily out-lines the stability criteria of the state-space manifold, defining the fluctuating higher dimensional black holes, with or without rotations. Indeed, we have shown, for a certain range of the parameters, that our intrinsic state-space geometric implications provide a unified framework to discuss both the statistical correlations, as well as the associated singularities and equilibrium configurations, which in general live on an arbitrary finitely curved intrinsic Riemannian manifold.

More precisely, we have analyzed a series of interesting examples, in order to illustrate the state-space geometry, which, as an intrinsic Riemannian geometry, may easily be defined from the negative Hessian matrix of the counting entropy of the associated rotating black hole configurations. In the concerned solutions, it has been shown that there exists an interesting set of scaling properties for the pair correlation functions. Thereby we have analyzed the stability on possible hyper-surfaces and related global properties of the state-space manifold of string theory and $M$-theory black holes. In particular, we have indicated that our ideas concerning macroscopic/ microscopic descriptions may easily examine the phase-space stability of underlying brane configurations, under the Gaussian fluctuations. It is thus possible to discuss the statistical implications, associated with an ensemble of boundary CFT microstates describing the particular black hole configurations, in chosen microscopic descriptions.

An important ingredient in the discussion of the state-space geometry turns out to be the fact that 
there exists a number of extremal black branes, non-extremal black branes, multi-centered black branes and the various other possible black brane configurations, inspiring certain general physical ramifications. Furthermore, it may be argued that the nature of state-space configurations remains the same under the possible subleading corrections. In fact, we find that the stability and critical properties of the considered black hole solutions remain intact in the large charge limit. The state-space geometry thus implied may have an interesting inter-relevance with the AdS/CFT correspondence. In particular one may try to come up with an adequate account for the correlated microstates and associated degeneracy of the fluctuating black hole configurations.

A parallel motivation may also be observed with certain logical inference of the string duality symmetries involving the parameters of the brane configurations and in particular, it turns out that the corresponding microscopic duality relations may easily be divulged by a set of decisive general coordinate transformations on the associated state-space manifold of the typical macroscopic solutions. Moreover, in the lieu of "thermodynamical/ statistical" behavior of the associated configurations, it appears that the significant parameters involving "macroscopic/ microscopic" relation(s) thus indicated may be in the close connection with those defining the "AdS/ CFT correspondence". Such an inspiration for the duality may in fact be envisaged via the associated degeneracy formula of underlying boundary conformal field theory, and thus it entangles only with the parameters of the configuration. Incrementally, there exists a series of interesting circumstances which support the fact that our study offers a geometrical approach to analyze the microscopic configuration of an ensemble of microstates which describe an equilibrium thermodynamic configuration, and thus one may actually apply it to divulge certain important physical and chemical behavior of the string theory/ M-theory black brane configurations.

In this paper, we specifically wish to consider a general situation of the higher dimensional configurations, where the state-space geometry determines the statistical pair correlation functions and correlation volume which may disclose certain critical nature of associated dual conformal field theory living on the boundary. Thus, one may exhibit distinguishing features of the microscopic duality acquisitions well-complied with the usual understanding of the associated parameters defining the concerned macroscopic configuration. In fact, it is well-known that both descriptions thus apprised may further be elucidated from the very possible application of the AdS/CFT correspondence [36. In the present consideration, we find that the state-space scalar curvature, as a function of the parameters, entails the central nature of the stabilities in the considered black hole solutions, while the determinant of the state-space geometry thus analyzed entangles with the notion of degeneracy of brane microstates constituting possible equilibrium statistical configurations.

Here, it is worth to mention that our intrinsic geometric formulation tacitly involves an underlying statistical basis, which for the rotating black hole configurations requires to chose an ensemble of CFT microstates in the thermodynamic limit and thus the interested formalization accommodates only the invariant parameters concerning the string theory or $M$-theory compactifications. In precise, this will imply that the state-space scalar curvature of rotating black brane configurations apprehends the nature of the correlation volume and thus the largest possible correlation being present in the underlying equilibrium microscopic systems. This strongly suggests that in the context of state-space manifold of the rotating black holes arising from a consistent string theory or $M$-theory solution when treated as closed systems, one may realize in both cases that the non-zero scalar curvature provides valuable information in analyzing the nature of quantum statistical interactions existing among the microstates of the considered brane configurations. Microscopically, as there exists many dual CFT descriptions, thus such brane configurations of interest may be anticipated as an ensemble of the bound states whose vacuum degeneracy takes an account of a large number of $D$-branes or an appropriate combination of dual $M$-branes.

\section{$3 \quad$ State-space Geometry}

In this section, we provide a brief account of the state-space geometry, which as an intrinsic Riemannian manifold $(M, g)$ serves our purpose, for describing the nature of statistical fluctuations in higher dimensional rotating black holes. Before focusing our attention on specific configurations, it is worthwhile reviewing some of the basics of intrinsic geometries, and then in general finding the state-space meanings, emerging from the asymptotic parameters of an ensemble of degenerate CFT microstates. For more details, see [58 [70] for certain black holes in general relativity, 71 in string theory, 38, for the recent account of the concerned notions in string theory and $M$-theory, and 72 75 for the chemical correlations and associated quark number susceptibilities in the 2- and 3-flavor hot QCD configurations.

In the present investigation, we shall provide detailed explanations for the state-space geometry, which analyzes fluctuations about an equilibrium ensemble of brane microstates, and there by describes the possible statistical configurations of the (rotating) black brane solutions. In particular, one may realize that our explorations shed light on the essential characteristics of the statistical correlations, quantum phase transitions and underlying critical behavior, if any, extant in the higher dimensional black brane configurations. The intrinsic Riemannian geometry, thus, turns out to be one of the main appliance, in order to study 
the state-space structures of the large class of black holes or black brane configurations being described in general relativity, as well as in the framework of fundamental string theories and $M$-theory.

First of all, let us recall that the usual probability distributions of thermodynamic fluctuations about an equilibrium statistical configuration characterize an invariant line interval for the corresponding intrinsic state-space geometry. Thus, by expanding the entropy about an equilibrium configuration, it turns out that the underlying probability distribution in the Gaussian approximation reads

$$
\Omega(x)=A \exp \left[-\frac{1}{2} g_{i j}(x) d x^{i} \otimes d x^{j}\right]
$$

where the pre-factor $A$ is the normalization constant of the Gaussian distribution under consideration, see [76] for the preliminary introduction and concerned physical motivations. In this case, one may easily deduce that the second moments of the fluctuations turn out to be defined by,

$$
\left\langle x_{i} x_{j}\right\rangle=\int x_{i} x_{j} \Omega(x) \sqrt{\|g\|} \prod_{i} d x_{i}, \forall i=0,1, . . n
$$

Therefore, it may easily be perceived in the critical set-up of 60] that the components of the inverse metric tensor are the second moments of the fluctuations, or the contravariant statistical pair correlation functions. Here, it turns out that the second moments of the concerned statistical fluctuations may in turn be given by,

$$
\left\langle x_{i} x_{j}\right\rangle=g^{i j}=<X^{i} X^{j}>
$$

where the state-space co-ordinates $\left\{X^{i}\right\}$ are conjugate to the intensive variables $\left\{x_{i}\right\}$, which may in particular be defined as,

$$
X^{i}:=\frac{\partial S(x)}{\partial x_{i}}
$$

Furthermore, one obtains that the underlying Gaussian distribution $\Omega(x)$ may be represented in terms of the conserved extensive charges $\left\{X^{i}\right\}$. In particular, it is easy observe that the Gaussian distribution describing our state-space geometry may be expressed as,

$$
P(X)=A \exp \left[-\frac{1}{2} g_{i j}(X) d X^{i} \otimes d X^{j}\right]
$$

Following 60,68, 69, it turns out that the natural inner product just mentioned may easily be ascertained, for an arbitrary $n$ parameter black brane configuration, and the concerned state-space turns out to be a n-dimensional intrinsic Riemannian manifold $M_{n}$. In particular, the associated entropy, as an embedding function, defines the covariant components of the metric tensor of the thermodynamic state-space geometry, which has originally been anticipated by Ruppeiner in the related articles 60 64. Here, we shall take this representation of the intrinsic geometry, and thus note that the covariant components of state-space metric tensor may be defined to be,

$$
g_{i j}:=-\frac{\partial^{2} S(\vec{X})}{\partial X^{i} \partial X^{j}}
$$

It is thus entirely clear from our outset, how this can be employed to describe the state-space geometry of black holes. However, if we restrict ourselves to the co-ordinates, which are extensive parameters, then one may be able to draw certain properties of the underlying fluctuations about an equilibrium statistical configuration. We may further urge that the variables $\left\{X^{i}\right\}$, associated with a set of asymptotic parameters carried by the higher dimensional black hole, define a suitable co-ordinate chart on the state-space manifold. In this representation, the coordinate $\vec{X}$ turns out to be a finite collection of the mass $M$, electricmagnetic charges $\left(P^{i}, Q_{i}\right)$, angular momenta $\left\{J_{i}\right\}$ and certain concerned local invariant charges $\left\{q_{i}\right\}$, if any. Thus, a most general co-ordinate on an arbitrary state-space manifold $(M, g)$ may be expressed as, $\vec{X}=\left(M, P^{i}, Q_{i}, J_{i}, q_{i}\right) \in M_{n}$.

With the the considerations developed in Appendix A, it is worth to perceive that the local stability of the underlying statistical configuration requires that the principal components of state-space metric tensor $g_{i i}$ signifying heat capacities should be positive definite

$$
\begin{aligned}
g_{Q Q} & >0 \\
g_{J J} & >0
\end{aligned}
$$

Moreover, we may inspect that the metric tensor for three parameters $P, Q, J$ comprises Gaussian fluctuations into the entropy as an embedding function and thus defines an intrinsic state-space manifold for the two charged rotating black brane configurations. This is because the components of the state-space metric tensor are related to the statistical pair correlation functions, which may be defined in terms of the three invariant parameters describing the microscopic conformal field theory on the boundary. Here, the simplest such 
examples which concern to the present analysis are the usual $D_{1}-D_{5}$ CFT configurations or the vacuum $M$-theory Kaluza-Klein black holes involving $D_{0}-D_{6}$-branes. Furthermore, it is worth to note that the local stability of the underlying statistical configurations requires that the principal components of the state-space metric tensor $g_{i i}$, which signify heat capacities, must be positive definite,

$$
\begin{aligned}
g_{Q Q} & >0 \\
g_{P P} & >0 \\
g_{J J} & >0
\end{aligned}
$$

In this case, the stability in the hyper-planes of the statistical configuration requires that all the off-diagonal fluctuations must vanish, as well. This implies that the consequent principal minors $\left\{p_{2}, p_{3}\right\}$ of the metric element should be strictly positive definite quantities on the entire state-space $\left(M_{3}, g\right)$. One thus finds that the hyper-planes stability criterion may rather be expressed as

$$
p_{2}:=\left|\begin{array}{ll}
g_{Q Q} & g_{Q P} \\
g_{Q P} & g_{P P}
\end{array}\right|>0
$$

Moreover, the existence of positivity of the state-space volume form imposes a stability condition on the Gaussian fluctuations that for the underlying statistical configurations one must have $p_{3}>0$. This in particular requires that the determinant of the state-space metric tensor must be positive definite as a function of electric-magnetic charges and angular momentum. In fact, a straightforward computation yields that the determinant of the metric tensor may be given to be,

$$
\begin{aligned}
\|g\| & =\frac{\partial^{2} S}{\partial P^{2}}\left(\frac{\partial^{2} S}{\partial Q \partial J}\right)^{2}+\left(\frac{\partial^{2} S}{\partial Q \partial P}\right)^{2} \frac{\partial^{2} S}{\partial J^{2}}+\left(\frac{\partial^{2} S}{\partial P \partial J}\right)^{2} \frac{\partial^{2} S}{\partial Q^{2}} \\
& -\frac{\partial^{2} S}{\partial P^{2}} \frac{\partial^{2} S}{\partial Q^{2}} \frac{\partial^{2} S}{\partial J^{2}}-2 \frac{\partial^{2} S}{\partial Q \partial P} \frac{\partial^{2} S}{\partial P \partial J} \frac{\partial^{2} S}{\partial Q \partial J}
\end{aligned}
$$

Consequently, it may be noticed that the scalar curvature corresponding to this state-space geometry elucidates the typical feature of Gaussian fluctuations about an equilibrium $D$-brane (or dual $M$-brane) microstates for possible two charge rotating configurations. Furthermore, we procure that it is easy to determine a global invariant on the three variable state-space manifolds. In this case as well, we nevertheless notice that one may actually explicate the nature of the underlying microscopic configurations. Just an interesting invariant which accompanies the information of correlation volume of underlying statistical systems turns out to be the intrinsic scalar curvature. A detailed analysis shows that the concerned scalar curvature may easily be determined

$$
R=\frac{1}{2\|g\|^{2}} f(Q, P, J)
$$

The exact expression for the $f(Q, P, J)$ is rather involved and we relegate it to the Appendix B. Furthermore, a systematic examination demonstrates that the state-space geometry with more charges and angular momenta can similarly be defined, and in turn, we must remark that one may easily deduce the statistical correlations and stability in terms of the corresponding ensemble of microstates which delicately characterize an elementary microscopic statistical basis against the macroscopic black brane configurations. Thence, it is not difficult to ponder from the theory of Gaussian distribution that the components of covariant state-space metric tensor may further be defined as the negative Hessian matrix of underlying entropy with respect to the mass, invariant charges and angular momenta, as well as certain possible exotic charges, if any, carried by the black brane configuration. It is worth to note in the case of present interest that the black holes, when viewed from out-side, may only be characterized by their mass, angular momenta, and electric-magnetic charges. Detailed internal structure and history of their formation are thus irrelevant in this "no hair" property. Such a drastic reduction of complexity is thus an important characteristic of the state-space investigations.

In the present description, the local stability condition of the underlying statistical configuration under the Gaussian fluctuations requires that all principal components of the fluctuations be positive definite, i.e. for given set of state-space variables $\left\{X^{1}, X^{2}, \ldots, X^{n}\right\}$ one must demand that $\left\{g_{i i}\left(X^{i}\right)>0 ; \forall i=1,2, \ldots, n\right\}$. In particular, it is important to note that this condition is not sufficient to insure the global stability of the chosen configuration and thus one may only accomplish a locally equilibrium configuration. It is however known that the complete stability condition requires that all principal components of the Gaussian fluctuations should be positive definite and the other components of the fluctuations should vanish [60]. In order to ensure this condition, one appraises that all the principal components and all the principal minors of the metric tensor must be strictly positive definite. This implies that the global stability condition constrains the allowed domain of the parameters of black hole configurations, which may interestingly be expressed by 
the following set of simultaneous equations:

$$
\begin{aligned}
p_{0} & :=1 \\
p_{1} & :=g_{11}>0 \\
p_{2} & :=\left|\begin{array}{ll}
g_{11} & g_{12} \\
g_{12} & g_{22}
\end{array}\right|>0, \\
p_{3} & :=\left|\begin{array}{lll}
g_{11} & g_{12} & g_{13} \\
g_{12} & g_{22} & g_{23} \\
g_{13} & g_{23} & g_{33}
\end{array}\right|>0, \\
\vdots & \\
p_{n} & :=\|g\|>0
\end{aligned}
$$

Strictly speaking, the determinant condition $p_{n}>0$ implies that there should exist a positive definite volume form on $\left(M_{n}, g\right)$. However, it is worth to notice that the classical thermodynamic fluctuation theory may not remain viable for the general black hole configurations, see for detail [60]. This is because a black hole cannot come into an equilibrium with any extensive, infinite environment, and in particular, for all asymptotic parameters the full Hessian determinant of the black hole entropy $S:=S\left(X^{i}\right)$ turns out to be negative definite, see for the related motivation [77. This is in fact required, in order to produce a local maximum in the total entropy of the configuration. Thus, the non-existence of the positive definite volume form on an intrinsic state-space manifold $\left(M_{n}, g\right)$ puts the full problem beyond the control of standard equilibrium fluctuation formalism, when all the state-space parameters $\left\{X^{i}:=\left(M, J_{i}, P^{i}, Q_{i}\right)\right\}$ are fluctuating.

Physically, the brane dynamics may ultimately favor extreme situations where the black hole may either completely evaporate or grow without limit. Moreover, the black hole will presumably create whatever particles or branes it likes near its event horizon, and an equilibrium is quite unlikely, until the environment is populated by the particles or branes in the same proportion to those created. However, in a limited domain of the parameters, when at least one of the parameter may slowly be changing in time, in comparison to the remaining ones, it obliges a class of stable configurations under the Gaussian fluctuations. This investigation thus implies a certain restricted class of stable configurations with arbitrary $m$ number of fluctuating parameters and the remaining $n-m$ parameters being effectively fixed or drifting very slowly out of the equilibrium configuration with the environment, where $1 \leq m \leq n$.

Interesting discussions of the phase transitions exist in literature which involve some change of the black hole horizon topology, 78, 79. However, there exists a large class of black holes with a spherical topology in the physical regime, which are quite energizing in their own, as we shall exhibit in the next section. However, we shall now analyze the behavior of collective state-space correlations, and in particular, we wish to illustrate certain peculiarities of the state-space geometry which in the large charge limit provide the correlation length for the associated microscopic configurations. Additionally, Ruppeiner has revived that the state-space scalar curvature remains proportional to the correlation volume $\xi^{d}$, where $d$ is the system's spatial dimensionality and $\xi$ is its correlation length which reveals related information residing in the microscopic models 61. Furthermore, the state-space curvature for higher dimensional black holes may be intertwined with vacuum phase transitions existing in the possible brane configurations and thus one may revel certain intriguing information of the possible microscopic CFTs.

Ruppenier has conjointly interpreted with the assumption "that all the statistical degrees of freedom of a black hole live on the black hole event horizon" and thus the scalar curvature signifies the average number of correlated Planck areas on the event horizon of the black hole [80. In particular, zero scalar curvature indicates certain bits of information on the event horizon fluctuating independently of each other, while the diverging scalar curvature signals a phase transition indicating highly correlated pixels of the informations. Moreover, Bekenstein has introduced an elegant picture for the quantization of the area of the event horizon being defined in terms of Planck areas [81, and in this concern it appears from our analysis that the microscopic resolution would possibly involve the framework of well-celebrated Mathur's fuzzballs [37]. Thus, our perspective discloses ground with the statements that the state-space scalar curvature of interest has not only exiguous microscopic knowledge of the black hole configuration, but in particular it has nice intrinsic geometric structures. Specifically, we find that the configurations under present analysis are effectively attractive in general, while they are stable only if at least one of the parameters remain fixed. Our hope is that finding statistical mechanical models with like behavior might yield further insight into the microscopic properties of black holes and conclusive physical interpretation of their state-space curvatures and related intrinsic geometric invariants.

With this general geometric prelude to the physics of a large class of higher dimensional rotating solutions, we shall now proceed to systematically analyze the state-space geometric structures characterized by a finite number of invariant electric-magnetic charges, angular momenta, mass, and other veritable parameters, if any, defining the microstates of the black hole configurations. 


\section{Rotating Black Hole Configurations}

In the present section, we shall first apply the notion of state-space geometry thus developed to the rotating Myers-Perry configurations. There after, we move on to investigate the related well-known two, three, charged rotating black hole configurations. We shall devote the present section to the possible descriptions and their interpretations against our intrinsic Riemannian geometry, which describes the vitality of an ensemble of equilibrium microstates of the extremely simple black holes. In particular, in the very first subsection we shall approach our study by analyzing the state-space manifold of the rotating black holes, which only comprises the mass and an angular momentum as invariant parameters, and then we shall proceed into the next subsections, which expose that the very close conclusions hold as well for the various higher charged rotating black hole configurations in the vacuum $M$-theory, string theory and possible supergravity theories.

In this spirit, we shall explain how the two point statistical correlation functions, as the function of parameters characterizing an ensemble of equilibrium microstates of the rotating black hole configurations, are well-structured in the framework of our state-space geometry. In turn, it shall be interesting to notice whether the correlation length of higher dimensional rotating configurations may be described as a regular or singular function of the charges and angular momentum on the concerned state-space manifolds. In both cases, we recognize from the perspective of state-space manifolds that it is not difficult to analyze the nature of the singularities, if any, present in the corresponding scalar curvature for the underlying microscopic configurations. In particular, we shall show that there may exist certain critical point(s), or critical line(s), or critical (hyper)-surface(s) on the state-space manifolds of the definite rotating black brane configurations on which the associated state-space scalar curvatures diverge, as a function of the parameters being specified by the considered configurations.

A main difficulty in assimilating the black hole solutions, however, appears to be somewhat anomalous. The concerned problem may be taken into account by the coordinate systems of the state-space manifold, in which the coordinates illuminating the abovementioned configuration take the simplest known form. Therefore, our conclusions thus accomplished would also be useful for obtaining adapted space-time parameters in the other possible settings. In the following, we shall study how the uniqueness theorems play a central role in the very possible study of generalized state-space manifolds encompassing a definite set of brane charges, concerning the higher dimensional rotating black hole configurations.

\subsection{Myers-Perry Black Holes}

This subsection, as the first exercise, elucidates the state-space geometry of five dimensional neutral black holes in the vacuum $M$-theory. We notice that these solutions are natural to be analyzed in the type- $I I$ string theory description. In particular, it is known that these neutral black holes carry a minimal set of quantum numbers, viz., mass and angular momentum which characterize the vacuum $M$-theory configurations. From the microscopic perspective, the intriguing idea emerges from the fact that these black holes may be appreciated by taking the product of $S^{1}$ with flat $T^{6}$, and then one may obtain the distinguishing $M$-theory solutions, whose type- $I I A$ reduction has $D_{0}$ and $D_{6}$ charges. Moreover, it has actually been shown in 16 that in a certain limit, akin to the decoupling limit in AdS/CFT, one may acquire the asymptotically flat neutral Myers and Perry black hole solutions [14. However, it is worth to mention from the type II $A$ dual standpoints that there are non-supersymmetric quadratically stable $D_{0}-D_{6}$ bound states $[82$, which serve as the basis for the microscopic picture of the statistical configurations.

Although supersymmetry turns out to be completely broken in this limit 83, nonetheless one may prove that, in the same limit, there are certain supersymmetric bound states of the constituent $D_{0}$ and $D_{6}$ branes 84. Indeed, it is stimulating to note that we are obliged to use two different sets of branes, and then, from the standpoints of type- II A string theory, the number of brane charges is doubled. In particular, this is reminiscent of the earlier suggestions that the neutral black holes should be viewed as a collection of branes and anti-branes 84. Moreover, there exists a mysterious duality invariant formula which, including the Schwarzschild black holes, reproduces the entropy of all known nonextremal black holes ascribed in terms of charges carried by the constituent branes and antibranes, see for details [84 85]. In both cases, there exist simple microscopic $D$-brane descriptions which precisely reproduce the Bekenstein Hawking entropy, which may thence be expressed in terms of the mass $M$ and angular momentum $J$ possessed by these extremal rotating black hole configurations in the vacuun $M$-theory. Specifically, one finds an illuminating expression for the Bekenstein Hawking entropy of the extremal Myers-Perry black holes, which may be expressed as

$$
S(M, J):=\frac{8}{3 G} \sqrt{\frac{2 \pi}{3}\left(G^{3} M^{3}-\frac{27}{32} \pi G^{2} J^{2}\right)}
$$

where $G$ shall henceforth denote the Newton coupling constant, see for details 14. In order to take a closer look at the state-space geometry arising from the equilibrium microstates of underlying $D$-brane 
configuration, we focus our attention on the negative Hessian matrix of the entropy of these simplest rotating Myers-Perry neutral black hole configurations. Here, it is worth to note that the mass and angular momentum are the unique parameters which characterize the Myers-Perry configurations, and consequently they form the co-ordinate chart for the underlying state-space geometry. Employing the previously proclaimed formulation, one may easily read off the components of the covariant state-space metric tensor to be

$$
\begin{aligned}
g_{M M} & =\sqrt{6 \pi} G^{2} M\left(\frac{9}{4} \pi G^{2} J^{2}-\frac{2}{3} G^{3} M^{3}\right)\left(G^{3} M^{3}-\frac{27}{32} \pi G^{2} J^{2}\right)^{-3 / 2} \\
g_{M J} & =-\frac{9}{8} \sqrt{6} \pi^{3 / 2} G^{4} J M^{2}\left(G^{3} M^{3}-\frac{27}{32} \pi G^{2} J^{2}\right)^{-3 / 2} \\
g_{J J} & =\frac{3}{4} \sqrt{6} \pi^{3 / 2} G^{4} M^{3}\left(G^{3} M^{3}-\frac{27}{32} \pi G^{2} J^{2}\right)^{-3 / 2}
\end{aligned}
$$

Within this framework, we observe that there exists a very simple description which divulges the geometric nature of the statistical pair correlations. The fluctuating extremal Myers-Perry black holes may thus easily be determined in terms of the mass and angular momentum of the underlying configurations. Moreover, it is evident that the principal components of the statistical pair correlations are positive definite for a range of the parameters of concerned black holes, which physically signifies a certain self-interaction of a fictitious particle moving on an intrinsic surface $\left(M_{2}(R), g\right)$. In particular, it is clear in this case that we have the following state-space metric constraints:

$$
\begin{aligned}
g_{M M} & >0, \forall(M, J) \mid \frac{27}{32} \pi J^{2}<G M^{3}<\frac{27}{8} \pi J^{2} \\
g_{J J} & >0, \quad \forall \text { admissible }(M, J)
\end{aligned}
$$

Consequently, we may easily reveal that the common domain of the above state-space constraints defines the range of physically sensible values of mass and angular momentum, such that the Myers-Perry black holes may remain into certain locally stable statistical configurations. We may also notice that the mass-mass component $g_{M M}$ of the state-space metric tensor is asymmetrical, in contrast to $g_{M J}$ and $g_{J J}$. This is physically be well-accepted, as the mass-mass component is somewhat like the head on collision of two equal mass particles, which alternate more energy in contrast to the other excitation, either involving a single rotating particle or a massless rotating (or spinning) particle. It is worth to point out that the relative pair correlation function determines the rotation parameter $(a:=J / M)$ of Myers-Perry black holes, which may be defined as the modulus of the ratio of mass-rotation to rotation-rotation statistical correlations. In particular, we procure that the rotation parameter thus apprised may be given as

$$
a:=\frac{3}{4}\left|\frac{g_{M J}}{g_{J J}}\right| .
$$

Moreover, the stability of Myers-Perry statistical configurations may earnestly be analyzed by computing the degeneracy of the associated two dimensional state-space manifold. In fact, we can easily ascertain that the determinant of the state-space metric tensor may be given to be

$$
g(M, J)=-3072 \pi^{2} G^{2} M^{4}\left(32 G M^{3}-27 \pi J^{2}\right)^{-2}
$$

The determinant of the metric tensor thus calculated is non-zero for any set of given non-zero mass and angular momentum, and thus it provides a non-degenerate state-space geometry for this configuration. In turn, one may illustrate the order of statistical correlations between the equilibrium microstates of the Myers-Perry rotating black hole system. Besides the fact that the principal components constraints $\left\{g_{i i}>\right.$ $0 \mid i=M, J\}$ imply that this system may accomplish certain locally stable statistical configurations, however the negativity of the determinant of the state-space metric tensor indicates that the underlying systems may globally endure unstable, as well. This connotes that there is no positive definite volume form on the $\left(M_{2}, g\right)$, and thus one may conclude that this system might go to some more stable brane configurations.

Furthermore, in order to examine certain global properties on these black holes phase-space configurations, one is required to determine the associated geometric invariants of the underlying state-space manifold. For the Myers-Perry black holes, the simplest invariant turns out to be the state-space scalar curvature, which may easily be computed by using the intrinsic geometric technology, defined as the negative Hessian matrix of the entropy captured by the rotating contributions. Explicitly, we discover that the state-space curvature scalar for the Myers-Perry configurations may easily be depicted to be

$$
R(M, J)=-\sqrt{\frac{27}{4 \pi}} G^{3}\left(32 G M^{3}-27 \pi J^{2}\right)^{-1 / 2}
$$

We recognize, in the framework of our state-space geometry, that the negative sign of the curvature scalar signifies that this system is effectively an attractive configuration under the Gaussian fluctuation. Thus, 
the Myers-Perry configurations are relatively less stable than the other configurations having certain nonnegative state-space scalar curvatures. Nevertheless, we observe that the curvature scalar thus considered is inversely proportional to the determinant of the underlying state-space metric tensor, In turn, we find that it remains a non-zero, finite, regular function of the mass and angular momentum carried by the Myers-Perry black holes.

It is however important to mention that the state-space geometric quantities may become ill-defined, if the state-space co-ordinates being defined as the space-time parameters, jump from one existing domain to the other domain of the ergo branch. This indicates that the Myers-Perry black holes correspond to certain interacting statistical configurations in a chosen ergo branch. In all admissible domain of the physical parameters, we discover that the rotating Myers-Perry black holes has no phase transition, and thus the fundamental statistical configurations are completely free from the critical phenomena.

Furthermore, we note, up to some easily appreciable constants $\alpha, \beta \in R$, that it is not difficult to determine the constant entropy curve and constant state-space scalar curvature curve for the rotating MyersPerry black hole configurations. In particular, we may apparently figure out that both the above possible curves, defined as real embedding functions, take a very simple form, which may simultaneously be given as

$$
M^{3}-\alpha J^{2}=\beta
$$

It thus turns out that the state-space quantities of the vacuum $M$-theory black holes, particularly for the neutral Myers-Perry configurations, which are far from being supersymmetric, may easily be analyzed by an appropriate combination of simple $D$-branes. However, it is known since the study of Strominger and Vafa [1] that the original string theory black hole solutions are both charged and supersymmetric. In particular, they have pointed out that (i) the charges of the black hole serve as tag marks which help to identify their microscopic constituents in string theory and (ii) the supersymmetry requirement constrains the phase-space configuration of these black holes. The next subsection would thus be devoted to investigate the notion of charges into our state-space geometry, and there by we shall analyze the possible role of concerned charges in the veritable black hole configurations in vacuum $M$-theory. In particular, we shall precisely compute the statistical pair correlations under this consideration, which ascribes certain convincing physical meaning to the state-space quantities for the simplest Kaluza-Klein configurations.

\subsection{Kaluza-Klein Black Holes}

In the present subsection, we shall study the state-space geometry arising from the two charged rotating Kaluza-Klein black hole solutions. In particular, we shall devote our attention to the possible descriptions, and their physical interpretations, of the state-space geometry arising from the vacuum $M$-theory black hole. A similar two charge system arises from the $D_{1}-D_{5}$ configurations, which we shall analyze in section 3.4. In both cases, we shall discuss certain vital issues of the underlying equilibrium microstates, which describe self and inter-brane statistical interactions, intertwined with the two charged rotating black holes. We find that one may easily examine the nature of the associated correlation lengths for these simple $D$-brane configurations.

We begin our discussion by examining the state-space quantities of the Kaluza-Klein black holes, which compound as well to the previously exposed properties of five-dimensional neutral Myers-Perry black holes. It is worth to mention that one may easily consider certain $M$-theory vacuum solutions, which describe the five-dimensional Kaluza-Klein black holes, and thus it appears interesting to find out their general interrelevance with those arising from the just encountered rotating Myers-Perry black holes. Here, the present perspective of the state-space geometry may thus interestingly be divulged in the extremal limit, which is akin to the decoupling limit of the underlying corresponding AdS/CFT correspondence.

We recommend [15] for detailed interpretations of these black hole solutions in ordinary four space-time dimensions. In this description, our framework displays that the state-space geometry of general KaluzaKlein black holes may be characterized by the mass $M$, angular momentum $J$, electric charge $Q$, and magnetic charge $P$. Here, we shall however focus our attention on the specific situations described in [86, 87, and in particular an interesting case emerges when the concerned parameters of the solution appease an inequality,

$$
2 G_{4} M \geq\left(Q^{2 / 3}+P^{2 / 3}\right)^{3 / 2}
$$

Moreover, we may realize that the state-space geometry of these black hole solutions measures an intriguing lower bound on the allowed angular momentum, than that arising from the limit of slow rotations satisfying $G_{4} J<P Q$. Our computation thus shows that these solutions are more strongly saturated than the constrained solutions in the extremal limit. It is worth to note that the observation of extremality and state-space conclusions are independent of the five dimensional rotations. First, we would like to signal a certain interesting behavior of the pair correlation functions for two charged black hole solutions in $\mathrm{M}$ 
theory, and then, from the view points of type IIA string theory, our analysis indicates that the state-space correlations may easily be described in the dual description of $D_{0}$ and $D_{6}$ brane cconfigurations.

Microscopically, the state-space geometry involves counting the microstates of a chosen configuration. The case of Kaluza-Klein solutions may be realized by taking the product of the Kaluza-Klein circle $S^{1}$ with $T^{6}$ with the volume $(2 \pi)^{6} V_{6}$. Thus, one may view them as vacuum solutions in M theory with $S^{1}$ being the $M$-theory compactification circle. Note, further, that our discussion may also begin by considering the usual coupling relation between the $M$-theory and IIA string theory, which may in particular be ascribed by the relation $R_{0}=g l_{s}$. Furthermore, the charges on the constituent $D_{0}$ and $D_{6}$-branes may be interpreted as the electric and magnetic charges, which respectively correspond to the net numbers $N_{0}$ and $N_{6}$ of the basic branes.

We focus our attention on the state-space analysis of five-dimensional vacuum $M$-theory configurations by considering the compact Kaluza-Klein circle of radius $R_{0}$, which is fibered over the two spheres of spherical symmetry of the underlying four dimensional black hole solutions. What follows here shows that the magnetic charges are quantized in terms of the Kaluza-Klein radius $R_{0}$, and that the electric charge renders as the inverse of the radius $R_{0}$ of the Kaluza-Klein circle. More precisely, the underlying electric and magnetic charges take integer values, in terms of the net number of constituent $D_{0}$ and $D_{6}$ branes. In turn, one arrives at the simple quantization condition that the existing charges may be inscribed as

$$
(Q, P)=\left(\frac{2 G_{4} N_{0}}{R_{0}}, \frac{N_{6} R_{0}}{4}\right)
$$

This is due to fact that the Kaluza-Klein circle bundles over $S^{2}$, which may in turn be labeled by an integer. Thus, the corresponding electric charges are also quantized, since they correspond to the momentum in the radial direction of the Kaluza-Klein circle. It turns out that the Kaluza-Klein black holes are described in a microscopic framework, whose CFT deals with $N_{0}$ number of $D_{0}$ branes and $N_{6}$ number of $D_{6}$ branes. There by, it is naturally interesting to peruse the investigation of underlying statistical configurations in this perspective. We therefore wish to analyze the state-space of two parameter extremal Kaluza-Klein black holes with an angular momentum $J$. Such space-time solutions appear quite naturally in the string theory, see for example [88. In this case, one finds that the underlying horizon entropy may be given to be,

$$
S\left(N_{0}, N_{6}, J\right):=\frac{A_{(4)}}{4 G_{4}}=2 \pi \sqrt{\frac{1}{4} N_{0}^{2} N_{6}^{2}-J^{2}}
$$

The state-apace geometry, constructed out of the equilibrium state of the rotating two charged Kaluza-Klein black holes resulting from the entropy, may now easily be computed, as earlier, from the negative Hessian matrix of the entropy, with respect to the brane numbers and angular momentum $\left\{N_{0}, N_{6}, J\right\}$, which form the coordinates of the intrinsic state-space manifold. Explicitly, we find that the components of the covariant metric tensor are given as,

$$
\begin{aligned}
g_{N_{0} N_{0}} & =4 \pi N_{6}^{2} J^{2}\left(N_{0}^{2} N_{6}^{2}-4 J^{2}\right)^{-3 / 2} \\
g_{N_{0} N_{6}} & =-\pi N_{0} N_{6}\left(N_{0}^{2} N_{6}^{2}-8 J^{2}\right)\left(N_{0}^{2} N_{6}^{2}-4 J^{2}\right)^{-3 / 2} \\
g_{N_{0} J} & =-4 \pi J N_{0} N_{6}^{2}\left(N_{0}^{2} N_{6}^{2}-4 J^{2}\right)^{-3 / 2} \\
g_{N_{6} N_{6}} & =4 \pi N_{0}^{2} J^{2}\left(N_{0}^{2} N_{6}^{2}-4 J^{2}\right)^{-3 / 2} \\
g_{N_{6} J} & =-4 \pi J N_{0}^{2} N_{6}\left(N_{0}^{2} N_{6}^{2}-4 J^{2}\right)^{-3 / 2} \\
g_{J J} & =4 \pi N_{0}^{2} N_{6}^{2}\left(N_{0}^{2} N_{6}^{2}-4 J^{2}\right)^{-3 / 2}
\end{aligned}
$$

Incidentally, we observe, from the simple $D$-brane description, that there exists an interesting brane interpretation, which describes the state-space correlation formulae arising from the corresponding microscopic entropy of the aforementioned non supersymmetric extremal black hole solutions. Furthermore, our statespace correlations turn out to be in a precise accordance with the underlying attractor configuration being disclosed in the special limit of Bekenstein-Hawking solutions. In the entropy representation, it may thus be noticed that the Hessian matrix of the entropy illustrates the nature of possible correlations between the set of extensive variables, which in this case are nothing more than the $D_{6}$ and $D_{0}$-brane charges and angular momentum. As mentioned before, we may articulate in this case again that, for all non-zero values of $N_{0}, N_{6}, J$, the principal components of intrinsic state-space metric tensor satisfy

$$
\begin{aligned}
g_{N_{0} N_{0}} & >0 \\
g_{N_{6} N_{6}} & >0 \\
g_{J} & >0
\end{aligned}
$$

Substantially, the principal components of state-space metric tensor signify heat capacities or the associated compressibility, whose positivity indicates that the underlying statistical systems are in local equilibrium, 
consisting of the $D_{6}$ and $D_{0}$-brane configurations. Furthermore, we perceive that the ratios of possible diagonal components vary as the inverse square, which weakens faster, and thus relatively quickly comes into an equilibrium configuration, than those involving the off diagonal ones. We further observe that the ratios of non-diagonal components vary inversely, and in turn remain comparable for a longer domain of the parameters varying under the Gaussian fluctuations. In particular, we easily inspect $\forall i \neq j \in\{0,6\}$ that the relative correlation functions satisfy the following simple relations:

$$
\begin{aligned}
\frac{g_{N_{i} N_{i}}}{g_{N_{j} N_{j}}} & =\left(\frac{N_{j}}{N_{i}}\right)^{2} \\
\frac{g_{N_{i} N_{i}}}{g_{J J}} & =\left(\frac{J}{N_{i}}\right)^{2} \\
\frac{g_{N_{i} J}}{g_{N_{j} J}} & =\frac{N_{j}}{N_{i}} \\
\frac{g_{N_{i} N_{i}}}{g_{N_{i} J}} & =-\frac{J}{N_{i}}
\end{aligned}
$$

Moreover, one should note that the behavior of the brane-brane correlation defined as $g_{N_{i}} N_{j}$ is asymmetric, in comparison with all other pair correlation functions. This may simply be understood by the fact that the brane-brane interaction imparts more energy than either the self-interaction or that due to the rotation. In particular, one discovers that the brane-brane relative pair correlation, with respect to the rotation-rotation correlation, reads

$$
\frac{g_{N_{0} N_{6}}}{g_{J J}}=-\frac{N_{0} N_{6}}{4}\left(1-8\left(\frac{J}{N_{0} N_{6}}\right)^{2}\right)
$$

We thus deduce that the relative brane-brane correlation vanishes exactly at half angular momentum of the vanishing entropy condition. This suggests that the brane-brane correlation is stable under the Gaussian fluctuations, if the four dimensional angular momentum satisfies an inequality, $|J|<\frac{N_{0} N_{6}}{2 \sqrt{2}}$. Nevertheless, our state-space geometry enjoys a lower bound on the attainable angular momentum, than the constraint arising from the acquainted slow rotations of the $M$-theory vacuum solutions. Furthermore, it may be inferred from our analysis, independently of the five dimensional rotations, that these solutions are more strongly saturated, than simply the condition of extremality, or the decoupling limit concerning the AdS/CFT.

In order to investigate certain global properties of the Kaluza-Klein configurations, we need to determine stability along each direction, each intrinsic plane, as well as on the full state-space manifold. In particular, for determining whether the underlying configuration is locally stable on state-space planes, one may compute the corresponding principal minor of the negative Hessian matrix of the entropy. In this case, we may easily appraise that the principal minor $p_{2}$, computed from the above state-space metric, reduces to

$$
p_{2}=\pi^{2} N_{0}^{2} N_{6}^{2}\left(12 J^{2}-N_{0}^{2} N_{6}^{2}\right)\left(N_{0}^{2} N_{6}^{2}-4 J^{2}\right)^{-2}
$$

Thus, for all physically possible values of brane charges, the minor constraint $p_{2}>0$ results into a restriction on the accessible values of the angular momentum. In particular, we may easily ascertain the nature of the state-space geometry of Kaluza-Klein systems, i.e. that the planar stability exists only in a limited domain of the configuration, whenever the angular momentum picks up at least $|J|=\frac{N_{0} N_{6}}{2 \sqrt{3}}$, or alternatively the planar stability requires that a given $D_{0}-D_{6}$-brane configuration is scarcely populated and thus the net brane charges are effectively bounded by the four dimensional rotation. Moreover, it is not difficult to investigate local stability in the full phase-space configuration, which may easily be carried out by computing the determinant of the state-space metric tensor. In this case, we observe that the determinant of the state-space metric tensor is

$$
g\left(N_{0}, N_{6}, J\right)=-4 \pi^{3} N_{0}^{4} N_{6}^{4}\left(N_{0}^{2} N_{6}^{2}-4 J^{2}\right)^{-5 / 2}
$$

which in turn never vanishes for any given non-zero brane charges. This non-degenerate metric tensor thus corresponds to a well-defined state-space, which may in fact solely be parameterized in terms of the $D$-brane charges, and an underlying angular momentum: $\left\{N_{0}, N_{6}, J\right\}$. We however emphasize that the determinant of the metric tensor does not take a positive definite form, and thus there is no positive definite volume form on the defined state-space $\left(M_{3}, g\right)$. It may thence be concluded that a Kaluza-Klein black hole, when considered as the bound state of $D_{0}-D_{6}$-branes in the type-II string description, does not correspond to an intrinsically stable statistical configuration.

Furthermore, in order to observe the nature of the global space-time properties, one can construct certain local identifications in the five-dimensional black hole configurations. In general, it turns out that 
the corresponding asymptotic spatial space-time geometry may globally be determined to be an orbifold $\mathbf{R}^{4} / \mathbf{Z}_{N_{6}}$ [16. Thus, the only configuration with $N_{6}=1$ causes globally asymptotically flat solutions, and all other possibilities with $N_{6}>1$ correspond to the black holes which sit at the tip of the conical space. It is worth to note that the horizon topology changes whenever $P \neq 0$, and thus the magnetic charge as an independent coordinate plays an important role in the realization of state-space geometry. In particular, one finds for the $N_{6}=1$ that the Kaluza-Klein-circle $S^{1}$ and the four dimensional spherical horizon $S^{2}$ combine into the topological $S^{3}$. At this point, we however discover that nothing special happens either to the statistical pair correlations, or to the determinant of the metric tensor, or to the state-space scalar curvature, as well for the choice of $N_{6}=1$, except for the fact that all associated constraints are now applied on the value of $N_{0}$. Furthermore, we may assert, from the duality invariance of the Kaluza-Klein entropy, that the analogous state-space conclusions remain true under the exchange of brane numbers, viz., $N_{0} \leftrightarrow N_{6}$.

Physically, it is interesting to explore the five-dimensional interpretation of the Kaluza-Klein solutions. In this case, it turns out, in the absence of a magnetic charge, that the horizon has a simple topology $S^{1} \times S^{2}$, where $S^{1}$ is the usual Kaluza-Klein circle. Thus, the space-time solution of interest turns out to be a boosted black string along the Kaluza-Klein direction. On other hand, the vanishing of electric charge $Q=0$ and $J=0$ divulges the extremal limit. In this case the considered space-time solution reduces to the Kaluza-Klein monopole, whose horizon geometry may in fact be described as a cigar space-time, fibered over the spherical horizon topology of the four dimensional solution. In the limits of either $\left\{J=0, N_{6}=0\right\}$ or $\left\{J=0, N_{0}=0\right\}$, we may easily apprise that the state-space geometry becomes an ill-defined intrinsic Riemannian manifold, and there is no interesting limit of the state-space scalar curvature. At this point, we strongly feel that the Gaussian approximation alienates down and we thus need to go beyond this consideration, in order to determine appropriately the long range behavior of the full statistical correlations.

However, we reveal, in the Gaussian approximation, that the statistical correlation volume, as a global invariant, takes an interesting form for the extremal microscopic configurations. Under these simplified identifications, it follows that the state-space scalar curvature takes an intriguing form, for the Kaluza-Klein black holes. In particular, it turns out that our geometric analysis does not entangle with the space-time orbifolds, unless the entropy changes its duality invariant form. Correspondingly, one may easily notice, in this limit, that the expression for the state-space scalar curvature may be given as

$$
R\left(N_{0}, N_{6}, J\right)=-\frac{1}{\pi N_{0}^{2} N_{6}^{2}}\left(N_{0}^{2} N_{6}^{2}+6 J^{2}\right)\left(N_{0}^{2} N_{6}^{2}-4 J^{2}\right)^{-1 / 2}
$$

The negative sign of the scalar curvature signifies that the underlying system is effectively an attractive configuration, and thus it is a less stable than an arbitrary positively curved configuration. Moreover, it is worth to mention that the scalar curvature thus determined never vanishes, for any physically affirmed value of the $D$-brane charges and angular momentum, and it is an everywhere regular function on the state-space manifold. Thus, for any non-zero brane charges, this system always corresponds to a weakly interacting statistical configuration.

Here, we find that the regular state-space scalar curvature seems to be comprehensively universal, for the given number of parameters of the configuration. In fact, the concerned idea turns out to be related with the typical form of the state-space geometry arising from the negative Hessian matrix of the duality invariant expression of the black hole entropy. As the standard interpretation, the state-space scalar curvature describes the nature of underlying statistical interactions of the possible microscopic configurations, which in particular turn out to be non-zero for the Kaluza-Klein black holes. Note that the absence of the divergences in the scalar curvature indicates that the present black hole solution is an everywhere thermodynamically stable system on the state-space configuration. Thus, it turns out that there are no any phase transitions or such critical phenomena in the underlying state-space manifold of the $D_{0}-D_{6}$-brane configurations. In fact, we may easily appreciate that the constant entropy curve is a standard curve, which may be given by

$$
N_{0}^{2} N_{6}^{2}=J^{2}+c
$$

where $c$ is some real constant, being determined from the given entropy expression. This determines the $D_{0}-D_{6}$ Kaluza-Klein black brane embedding in the view-points of state-space geometry. Moreover, we may also disclose in the present case that the curve of constant scalar curvature is given as,

$$
\left(N_{0}^{2} N_{6}^{2}+6 J^{2}\right)^{2}=k^{2} N_{0}^{4} N_{6}^{4}\left(N_{0}^{2} N_{6}^{2}-4 J^{2}\right),
$$

where $k$ is some real constant which may easily be fixed of the given value of the scalar curvature. Moreover, it is not difficult to enunciate that the quantization condition existing on the electric and magnetic charges signifies a general coordinate transformation on the state-space manifold, which may be presented in terms of the net numbers of respective branes. This in turn yields that the brane charges may be written as, $Q=2 G_{4} M_{0} N_{0}$ and $P=2 G_{4} M_{6} N_{6}$, where $G_{4}$ is the usual four dimensional Newton's coupling constant 
given by $G_{4}=g^{2} l_{s}^{8} / 8 V_{6}$. In this concern, the article [16] implies that the masses of the individual $D_{0}$, $D_{6}$-branes may be expressed as

$$
\begin{aligned}
& M_{0}=\frac{1}{g l_{s}} \\
& M_{6}=\frac{V_{6}}{g l_{s}^{7}}
\end{aligned}
$$

This suggests that the state-space fluctuations thus described may as well be expressed in terms of the individual brane masses and the four dimensional rotation. In particular, it is interesting to reveal how our state-space geometry, expressed in terms of the $\left\{M_{0}, M_{6}, J\right\}$, entangles with the corresponding ADM mass, $M=\left[\left(M_{0} N_{0}\right)^{2 / 3}+\left(M_{6} N_{6}\right)^{2 / 3}\right]^{3 / 2}$ of the extremal Kaluza-Klein black holes. Thus, it may be plausible to describe possible energy excitations in the $D_{0}-D_{6}$ microscopic Kaluza-Klein configurations. More precisely, the four-dimensional mass is dominated by the mass of the Kaluza-Klein monopole and, in this limit, the excitation energy above the Kaluza-Klein monopole is equal to the ADM mass of the five-dimensional black hole. Here, it may once again be seen from [16 that the electric-magnetic charges of the Kaluza-Klein black holes may be determined by the five dimensional angular momenta $\left\{J_{1}, J_{2}\right\}$ and the four-dimensional angular momentum $J$ via the relations

$$
\begin{aligned}
& N_{0}\left(J_{1}, J_{2}, J\right):=4 J^{2} \frac{J_{1}+J_{2}}{\left(J_{1}-J_{2}\right)^{2}} \\
& N_{6}\left(J_{1}, J_{2}, J\right):=\frac{J_{1}-J_{2}}{2 J}
\end{aligned}
$$

This eases us to describe our state-space construction in term of the two five dimensional momenta and a customary four dimensional angular momentum. Here, we reveal that the set $\left\{J_{1}, J_{2}, J\right\}$ forms another coordinate chart for the state-space manifold of the rotating Kaluza-Klein black holes. It has further been explained in [16] that, if the size of the black hole is much smaller than the Kaluza-Klein compactification radius, then the finite-size effects become rather negligible, and one recovers the neutral five-dimensional Myers-Perry black holes. Our investigations thus attribute a unified framework, for analyzing the various possible representations and specifications of the statistical fluctuation, underlying in the vacuum $M$-theory black hole configurations.

Furthermore, there might exist various possible microscopic descriptions of the state-space correlations, what we have been invoking may efficiently be exploited from the view-points of counting the microstates for supersymmetric four-dimensional black holes with a different choice of large charges [89. This observation is based on the related U-dualities, ensuring that the $D_{3}$-branes contain precisely the right number of states to reproduce the leading order entropy of vacuum $M$-theory black holes. This goes with the understanding that the macroscopic entropy of these black holes remains independent of the moduli fields arising from the compactification. Here, it is worth to note that the corresponding statistical pair correlations seem to be solely accomplished from those states that are associated with the intersection point of the branes, see for further details 89 .

Moreover, Taylor has shown 82 that there exists certain interesting $D$-brane configurations, for which the bound state of four $D_{0}$-branes and four $D_{6}$-branes may be described by the gauge theory configuration living on the worldvolume of the $D_{6}$-brane. Therefore, one may urge that the state-space geometric revelation of the possible configurations lies on the fact that the T-duality along the three cycles of the torus makes this configuration equivalent to that of the four $D_{3}$-branes. As invoked earlier, we may notice that the other string duality relations may also be realized as the general coordinate transformations on the concerned state-space manifold.

The other possibility materializing, in the present context of our state-space geometry, goes with an understanding of the Kaluza-Klein black holes directly in the $M$-theory, possibly in terms of gravitons and perhaps branes, or in terms of $D_{6}$-branes with a certain flux corresponding to $D_{0}$-branes, rather than comporting directly with the $D_{3}$-brane configurations. In particular, the quest of state-space manifold under consideration corresponds to an interesting intrinsic geometric problem, against the counting of the number of certain instantons in the six-dimensional Yang-Mills theory (see 90 for the D-instanton contributions to the graviton scattering amplitudes and related $S U(N)$ Yang-Mills instanton corrections).

\subsection{Supersymmetric $A d S_{5}$ Black Holes}

In this subsection we analyze state-space manifold of supersymmetric $A d S_{5}$ black holes arising in typeIIB string theory compactifications. In the framework of fractional-BPS supergravities, we shall exploit the fact that the $\frac{1}{16}$-BPS solutions possess a characteristic property that all the physical charges are not independent. We observe that the state-space geometry emerging from the Gaussian fluctuations of the Bekenstein-Hawking entropy admits a remarkably simple expression, in terms of the exclusive real charges 
and self dual angular momentum, which may intimate its microscopic origin via the Cardy formula, or the universal Hardy-Ramanujan formula, determining the degeneracy of microstates. We further point out that the physical properties of underlying state-space correlations achieve notably illuminating forms, which simply emerge, due to fact that there are certain upper bounds for the angular momenta. The concerned constraints may be furnished in terms of the electric charges which describe the possible maximum rotation for the supersymmetric $A d S_{5}$ black hole configurations.

From the verge of supergravity considerations, it turns out that the bosonic field contents of supergravity theories are fundamentally the space-time metric tensor $g_{\mu \nu}$, the graviphoton 9194 and the $n-1$ vector fields, which may thence be composed together as $A^{i}(i=1, \cdots, n)$, and the $n-1$ real scalars $\phi^{a}(a=$ $1, \cdots, n-1)$, see for details 95 . It has interestingly been visualized, in the case of the $U(1)^{3} \subset S O(6)$ truncation, that the $\mathcal{N}=4$ gauged supergravity may be embedded into type-IIB string theory [96]. In the present case, one finds that the $A d S_{5} \times S^{5}$ configuration thus specified has three vector fields, a tensor field $C_{123}$ and three constrained scalars $X^{i}$, which measure the squashing of $S^{5}$ taking equal vacuum values, which in turn make the internal $S^{5}$ to be a smooth round sphere. Furthermore, it is possible to check that the insertions of these values admit an intriguing physical appreciation for the supergravity black holes in $A d S_{5} \times S^{5}$ solutions. Thus, one may easily divulge the state-space manifold arising from the BekensteinHawking entropy of supersymmetric $A d S_{5}$ black holes.

As we have already mentioned the black holes of present interest carry two angular momenta in $A d S_{5}$, viz., $J_{1}$ and $J_{2}$, as well as they endure three $U(1)^{3} \subset S O(6)$ charges, say $Q_{i}(i=1,2,3)$. However, there exists a unique feature of these supersymmetric $A d S_{5}$ black holes configuration, i.e. that the associated physical charges are not all independent. Thus, these solutions may not always be regular, see for example [26, 97, 98. However, similar notions hold for the general supersymmetric $A d S_{n}$ black holes in minimal gauged supergravities. Although there exists an ambiguity in the entropy expression, it may however be defined in terms of the dependent physical charges. In order to compare it with the microscopic descriptions, there indeed exist certain implicit prescriptions in the literature [33, 34]. In turn, Kim et. al. observed, in certain specific regimes of the parameters, that the underlying entropy for the symmetric supergravity configurations admits a remarkably simple expression, in terms of the reliant physical charges

$$
S\left(Q_{1}, Q_{2}, Q_{3}, J\right)=2 \pi \sqrt{Q_{1} Q_{2}+Q_{2} Q_{3}+Q_{1} Q_{3}-N^{2} J}
$$

where $J=\frac{J_{1}+J_{2}}{2}$ is the self-dual part of the angular momenta, see for details [35]. Thus, the intrinsic Riemannian geometry, as the equilibrium state-space configuration of the three charged extremal rotating black hole resulting from the concerned fractional BPS-corrected entropy, may immediately be computed, as earlier, from the negative Hessian matrix of the entropy. We find that the components of the state-space metric tensor may easily be obtained, with respect to the underlying electric charges and the self-dual angular momentum, as

$$
\begin{aligned}
g_{Q_{1} Q_{1}}= & \frac{\pi}{2}\left(Q_{2}+Q_{3}\right)^{2}\left(Q_{1} Q_{2}+Q_{2} Q_{3}+Q_{1} Q_{3}-N^{2} J\right)^{-3 / 2} \\
g_{Q_{1} Q_{2}}= & -\frac{\pi}{2}\left(Q_{1} Q_{2}+Q_{2} Q_{3}+Q_{1} Q_{3}-Q_{3}^{2}-2 N^{2} J\right) \\
& \left(Q_{1} Q_{2}+Q_{2} Q_{3}+Q_{1} Q_{3}-N^{2} J\right)^{-3 / 2} \\
g_{Q_{1} Q_{3}}= & -\frac{\pi}{2}\left(Q_{1} Q_{2}+Q_{2} Q_{3}+Q_{1} Q_{3}-Q_{2}^{2}-2 N^{2} J\right) \\
& \left(Q_{1} Q_{2}+Q_{2} Q_{3}+Q_{1} Q_{3}-N^{2} J\right)^{-3 / 2} \\
g_{Q_{1} J}= & -\frac{\pi}{2} N^{2}\left(Q_{2}+Q_{3}\right)\left(Q_{1} Q_{2}+Q_{2} Q_{3}+Q_{1} Q_{3}-N^{2} J\right)^{-3 / 2} \\
g_{Q_{2} Q_{2}}= & \frac{\pi}{2}\left(Q_{1}+Q_{3}\right)^{2}\left(Q_{1} Q_{2}+Q_{2} Q_{3}+Q_{1} Q_{3}-N^{2} J\right)^{-3 / 2} \\
g_{Q_{2} Q_{3}}= & -\frac{\pi}{2}\left(Q_{1} Q_{2}+Q_{2} Q_{3}+Q_{1} Q_{3}-Q_{1}^{2}-2 N^{2} J\right) \\
g_{Q_{2} J}= & -\frac{\pi}{2} N^{2}\left(Q_{1}+Q_{3}\right)\left(Q_{1} Q_{2}+Q_{2} Q_{3}+Q_{1} Q_{3}-N^{2} J\right)^{-3 / 2} \\
g_{Q_{3} Q_{3}}= & \frac{\pi}{2}\left(Q_{1}+Q_{2}\right)^{2}\left(Q_{1} Q_{2}+Q_{2} Q_{3}+Q_{1} Q_{3}-N^{2} J\right)^{-3 / 2} \\
g_{Q_{3} J}= & -\frac{\pi}{2} N^{2}\left(Q_{1}+Q_{2}\right)\left(Q_{1} Q_{2}+Q_{2} Q_{3}+Q_{1} Q_{3}-N^{2} J\right)^{-3 / 2} \\
g_{J J}= & \frac{\pi}{2} N^{4}\left(Q_{1} Q_{2}+Q_{2} Q_{3}+Q_{1} Q_{3}-N^{2} J\right)^{-3 / 2}
\end{aligned}
$$

This framework thus affirms that there exists a lucid geometric enumeration, which describes the possible statistical pair correlations, determined in terms of the charges and angular momentum of the supersymmetric $A d S_{5}$ black holes. Hitherto, it is apparent that the principal components of the statistical pair correlations 
are positive definite, for the whole allowed range of concerned invariant parameters of the $A d S_{5}$ black holes. In particular, it may easily be observed that the following state-space metric constraints are satisfied

$$
\begin{aligned}
g_{Q_{1} Q_{1}} & >0 \forall\left(Q_{1}, Q_{2}, Q_{3}, J\right) \mid Q_{2} \neq-Q_{3} \\
g_{Q_{2} Q_{2}} & >0 \forall\left(Q_{1}, Q_{2}, Q_{3}, J\right) \mid Q_{3} \neq-Q_{1} \\
g_{Q_{3} Q_{3}} & >0 \forall\left(Q_{1}, Q_{2}, Q_{3}, J\right) \mid Q_{1} \neq-Q_{2} \\
g_{J} & >0 \forall \text { admisible }\left(Q_{1}, Q_{2}, Q_{3}, J\right) \mid N \neq 0
\end{aligned}
$$

Physically, we may notice that the principal components of the state-space metric tensor $\left\{g_{Q_{i} Q_{i}}, g_{J J} \mid i=\right.$ $1,2,3\}$ signify certain heat capacities (or the associated compressibilities), whose positivity exhibits that the underlying system is in local equilibrium configurations. Our analysis further complies that the positivity of $g_{J}$ obliges that the associated holographic conformal field theory living on the boundary must prevail a non vanishing value of the central charge.

Interestingly, it is worth to note that our geometric expressions arising from the black hole entropy show that either one of the three electric charges may safely be turned off, say $Q_{i}=0$, while having a well-defined state-space geometry. However, it is unfeasible to have an intrinsic $A d S_{5}$ black hole state-space configuration with only a single electric charge, say $Q_{i}=Q_{j}=0$, since the objects inside the square-root of the statistical correlations cannot be any more well-defined real positive definite quantities.

Furthermore, the ratio of the principal components of statistical pair correlations varies as an inverse square of the addition of two other charges; while that of the other off diagonal correlations only vary inversely. In particular, it is not difficult to inspect, for non-identical $i, j, k \in\{1,2,3\}$, that the statistical pair correlations are consisting of the following scaling properties:

$$
\begin{aligned}
\frac{g_{Q_{i} Q_{i}}}{g_{Q_{j} Q_{j}}} & =\left(\frac{Q_{j}+Q_{k}}{Q_{i}+Q_{k}}\right)^{2} \\
\frac{g_{Q_{i} Q_{i}}}{g_{J} J} & =\left(\frac{Q_{j}+Q_{k}}{N^{2}}\right)^{2} \\
\frac{g_{Q_{i} J}}{g_{Q_{j} J}} & =\frac{Q_{j}+Q_{k}}{Q_{i}+Q_{k}} \\
\frac{g_{Q_{i} Q_{i}}}{g_{Q_{i} J}} & =-\frac{Q_{j}+Q_{k}}{N^{2}} \\
\frac{g_{Q_{i} Q_{j}}}{g_{Q_{k} J}} & =\frac{\left(Q_{1} Q_{2}+Q_{2} Q_{3}+Q_{1} Q_{3}-2 N^{2} J\right)-Q_{k}^{2}}{N^{2}\left(Q_{i}+Q_{j}\right)} \\
\frac{g_{Q_{i} Q_{j}}}{g_{Q_{i, j} Q_{k}}} & =\frac{\left(Q_{1} Q_{2}+Q_{2} Q_{3}+Q_{1} Q_{3}-2 N^{2} J\right)-Q_{k}^{2}}{\left(Q_{1} Q_{2}+Q_{2} Q_{3}+Q_{1} Q_{3}-2 N^{2} J\right)-Q_{j, i}^{2}}
\end{aligned}
$$

The concerned scaling investigations may further be strengthened by the fact that the self-dual angular momentum $J$ of the $A d S_{5}$ black holes is constrained from above by the electric charges $Q_{i}$ by the bound: $J_{1}+J_{2} \leq \frac{2}{N^{2}}\left(Q_{1} Q_{2}+Q_{2} Q_{3}+Q_{3} Q_{1}\right)$, see for instance [27]. Here, our analysis thus demonstrates that the positivity of state-space correlations among the non-identical $U(1)$ charges $\left\{Q_{k} \mid k=1,2,3\right\}$ stipulates a modified lower bound for the self dual angular momentum

$$
J \leq \frac{2}{N^{2}}\left(Q_{1} Q_{2}+Q_{2} Q_{3}+Q_{1} Q_{3}-Q_{k}^{2}\right)
$$

This is because the brane-brane pair correlations involve the other remaining $U(1)$ charges of the underlying configuration. Thus one establishes, for equal value of charges, that the bound arising from the reality of the entropy is only three quarters of the stability criteria of the pair correlations between the non-identical electric charges.

Apart from the positivity of principal components of the state-space metric tensor, one demands that all the associated principal minors should be positive definite, in order to accomplish the local stability. It is nevertheless not difficult to compute the principal minors of the associated Hessian matrix of the entropy concerned with the spinning $A d S_{5}$ black holes. In fact, after some simple manipulations one encounters that the local stability conditions on the two dimensional surfaces and three dimensional hypersurfaces of the state-space manifold may respectively be measured by the following relations:

$$
\begin{aligned}
& p_{2}=\pi^{2}\left(N^{2} J+Q_{3}^{2}\right)\left(Q_{1} Q_{2}+Q_{2} Q_{3}+Q_{1} Q_{3}-N^{2} J\right)^{-2} \\
& p_{3}=2 \pi^{3} N^{2} J\left(Q_{1} Q_{2}+Q_{2} Q_{3}+Q_{1} Q_{3}-N^{2} J\right)^{-5 / 2}
\end{aligned}
$$


For all physically allowed values of invariant charges of the $A d S_{5}$ black holes, we thus stipulate that the minor constraint $p_{2}>0$ obliges that the domain of the ascribed angular momentum must respectively be greater than $J=-\left(Q_{3} / N\right)^{2}$, while the constraint $p_{3}>0$ imposes that the angular momentum must be a positive definite real number. In particular, we may easily inspect the nature of the state-space geometry for the spinning $A d S_{5}$ black holes, i.e. that the planar and hyper-planar stabilities of the system together demand just the existence of an arbitrary positive value of the self-dual angular momentum.

In addition, it is likewise evident that the local stability of the full phase-space configuration may be determined by computing the determinant of the concerned state-space metric tensor. Here, we may easily provide a compact formula, for the determinant of the metric tensor. In particular, our intrinsic geometric analysis assigns the following expression, for the determinant of the metric tensor, as the function of various possible values of electric charges and the angular momentum:

$$
g\left(Q_{1}, Q_{2}, Q_{3}, J\right)=-\pi N^{4}\left(Q_{1} Q_{2}+Q_{2} Q_{3}+Q_{1} Q_{3}-N^{2} J\right)^{-3}
$$

The determinant of the underlying metric tensor thus remains non-zero for the non-vanishing central charge of the corresponding dual holographic $\mathcal{N}=4$ Yang-Mills theory. This indicates that the state-space geometry is non degenerate at extremality, as a consequence of the higher derivative central charge contributions. Note however that the determinant of the metric tensor does not admit a positive definite form in the large charge limit, and thus there is no positive definite volume form on the intertwined state-space $\left(M_{4}, g\right)$. This is indeed justifiable from the fact that the equilibrium entropy approaches its maximum value, while the same may not remain valid in the possible hyper-planes of the concerned state-space configurations. It may further be noted in this description that the spinning $A d S_{5}$ black holes do not fabricate an intrinsically stable statistical configuration. Thus, it is possible that the underlying microscopic states may smoothly shift into a more stable ensemble of brane configurations.

Although the appraised expression of the entropy may not be unique, due to an existence of the relation between the physical charges $Q_{i}$ and angular momentum $J$ for the allowed black hole solutions. However, in the small black hole regime, where the solutions allow $Q_{i} \ll N^{2}$, the underlying entropy can be transcribed into a perturbative form, for which we emphasize that there are indeed interesting perspectives for the statespace geometry of the $A d S_{5}$ configurations. Such a perturbative manifestation of the supersymetric black hole entropy has in particular been advocated to meet the microscopic aspects of $A d S_{5}$ black holes, see for details [33. Eventually, our analysis discloses that the determinant of the metric tensor takes a small positive definite value. Thus, one may realize an intrinsically stable statistical configuration, with the existence of the globally defined positive definite volume form on the state-space manifold $\left(M_{4}, g\right)$. Therefore, the statespace configuration, in the limit of vanishing angular momentum or infinite central charge of the holographic CFT, inclines towards a degenerate intrinsic Riemannian manifold.

In order to conclusively analyze the nature of the interaction and the other concerned properties of the statistical configurations, one needs to determine certain global invariants on the parametric state-space manifold $\left(M_{4}, g\right)$. One may in fact ascertain that such a simplest invariant is the state-space scalar curvature, which may now be computed in a straightforward fashion by applying our previously advertised intrinsic geometric technology. The explicit expression for the scalar curvature is quite simple, and we notice that it may easily be expressed as

$$
R\left(Q_{1}, Q_{2}, Q_{3}, J\right)=-\frac{9}{4 \pi}\left(Q_{1} Q_{2}+Q_{2} Q_{3}+Q_{1} Q_{3}-N^{2} J\right)^{-1 / 2}
$$

Here, it is worth to mention that the scalar curvature is a regular function of the electric charges and angular momentum. In the large charge limit, in which the asymptotic expansion of the concerned BPS-entropy calculation is valid, it inclines to a vanishingly small value. The negative sign of the state-space scalar curvature discloses that the underlying configuration is effectively an attractive system. Thus, the spinning $A d S_{5}$ black holes turn out to be less stable than an arbitrary positively curved state-space configuration. In addition, this is due to the fact that the phase-space configuration of supersymmetric solutions is drastically constrained and is subject to powerful non-renormalization theorems and state-counting techniques. Moreover, it is important to remember that the scalar curvature thus determined never vanishes, for any physically affirmed value of the invariant charges and angular momentum, and is an everywhere regular function on the state-space manifold, unless either one of the electric charge approaches infinity. Thus, for any non-zero value of the charges and spinning angular momentum, the $A d S_{5}$ black hole always corresponds to a weakly interacting statistical configuration. Furthermore, it is not difficult to check that both the constant entropy and constant state-space scalar curvature curves take the same form

$$
Q_{1} Q_{2}+Q_{2} Q_{3}+Q_{1} Q_{3}=N^{2} J+b_{i},
$$

Such definite bound on a collection of states in itself is not surprising for the spinning black holes, as the microscopic degeneracy depends on the ensemble of microstates, while the corresponding statistical correlations 
depend on the state-space configuration. The concerned bound of the degeneracy of the microstates may in turn be obtained from the central charge of an underlying four dimensional dual holographic conformal field theory. Consequently, it is not difficult to manipulate that the constants $b_{i}$ may respectively be defined as

$$
\begin{aligned}
b_{1} & :=\frac{k_{1}^{2}}{4 \pi^{2}} \text { for constant entropy } \\
b_{2} & :=\frac{81}{16 \pi^{2} k_{2}^{2}} \text { for constant scalar curvature }
\end{aligned}
$$

where $\left(k_{1}, k_{2}\right)$ are the chosen values of the entropy and scalar curvature for the state-space $\left(M_{4}, g\right)$. In order to understand the origin of the statistical correlations underlying these objects, it will also be interesting to consider an exclusive microscopic sector of these configurations and then try to investigate the statespace quantities for the supersymmetric $A d S$-black holes. The purpose of this investigation may thus be to explore the macroscopic and microscopic aspects of the $\frac{1}{16}$-BPS objects, hoping that our geometric results would provide important clues for future works giving an account on both sides: for instance, the statespace correlations defining an intrinsic Riemannian geometry, arising from the Bekenstein-Hawking entropy formula, and that in the counting degeneracy of the $A d S$-black hole microstates. For the other possible attempts to analyze thermodynamics and the BPS aspects of the supersymmetric black holes, see [33.34, 39.

Decisively, our state-space geometric expressions which are exact in all regime of charges, exhibit certain interesting comparisons and definite agreements with the expressions advocated in previous achievements. The angular momentum factor originating from the central charge of the $\mathcal{N}=4$ Yang-Mills theory, which is holographically dual to the string theory in $A d S_{5} \times S^{5}$, governs the nature of the correlation length of the underlying statistical systems. In particular, it is worth to indicate that the prefactor, $N^{2}=4 c$, where $c$ signifies the central charge of the $\mathcal{N}=4$ Yang-Mills theory, determines the typical characteristic of the fundamental microscopic theory. The symmetric supergravity condition requires that $c$ should satisfy $c=\pi l^{3} / 8 G$. Here, the internal nature of the $A d S_{5}$ length parameter $l$ connotes the underlying microscopic objects, whether they are giant gravitons or wrapped branes [26]. Our state-space expressions thus obtained are indeed remarkably simple and allude that there should exist appropriate microscopic/macroscopic explanations for the statistical correlations for these configurations. The observations may thus be a motivation factor to further investigate how the Cardy or Hardy-Ramanujan formula sets down certain microscopic explanations for ascertaining similar examinations to the present, as well as to the other possible supergravity solutions.

The points in question may nevertheless be addressed for the rotating objects in Minkowski space, as well. In order to appraise this point more clearly, it turns out very interesting to consider well studied $D_{1}-D_{5}$ configurations. In particular, we wish to visualize how the state-space correlators behave when such an underlying BPS solution saturates the angular momentum bound. For instance, the entropy of rotating $D_{1}-D_{5}$ on $T^{5}$ (or $K_{3}$ ) may be given by $S=\gamma \pi \sqrt{Q_{1} Q_{5}-J}$, where $\gamma$ depends on the chosen compactification. Moreover, it turns out that the zero entropy limit of these configurations may be consigned by the circular supertubes [100 103], or U-duals of them 39 104 106]. In the lieu of $A d S_{5}$ black holes state-space geometry, it may thus be intriguing to examine the state-space correlators for a 2-charge black holes, whose degeneracy formula defines an appropriate simple expression for the microscopic entropy, in large charge limit. Therefore, we shall devote the next subsection to a detailed analysis of these configurations. In particular, we would like to allocate the state-space geometric meaning of the $N^{2}$ factor, which makes the problem more challenging, from the view points of $D_{1}-D_{5}$ CFT.

\section{$4.4 \quad D_{1}-D_{5}$ Configurations}

In this subsection, we shall analyze the state-space geometry of the most exhaustively contemplated two charged black brane configurations, which naturally emerge from the equilibrium microstates of various interesting bubbling supergravity solutions, see for details 100 103. It turns our that one may easily ascribe the state-space definitions to the central charge contributions associated with the rotating black holes in Minkowski space, and in turn we may focus our attention on the possible U-dual configurations as well. The concerned details of the intriguing solutions have been described in 39, 104 106]. Here, the purpose is to exploit the state-space meanings of the $N^{2}$ factor arising from an elementary four dimensional holographic conformal field theory living on the boundary [35. As we have encountered the state-space geometry of the $A d S_{5}$ black holes in the previous subsection, this subsection analyzes the state-space fluctuations for the restricted 2-charge $D_{1}-D_{5}$ rotating solutions in the extremal limit, and there by examines the inclusion of a holographic central charge contribution. It turns out that the involved entropy may be defined via an appropriate degeneracy formula to be

$$
S\left(Q_{1}, Q_{5}, J\right):=2 \pi \sqrt{Q_{1} Q_{5}-N^{2} J}
$$


The state-space geometry describing the correlations between the equilibrium microstates of the two charged rotating extremal $D_{1}-D_{5}$ black holes, resulting from the degeneracy of the microstates may easily be computed, as earlier, from the Hessian matrix of the entropy, with respect to the $D_{1}, D_{5}$ charges, viz,, $Q_{1}, Q_{5}$ and the angular momentum $J$. At this juncture, we obtain that the components of the underlying state-space covariant metric tensor are given by

$$
\begin{aligned}
g_{Q_{1} Q_{1}} & =\frac{\pi}{2} Q_{5}^{2}\left(Q_{1} Q_{5}-N^{2} J\right)^{-3 / 2} \\
g_{Q_{1} Q_{5}} & =-\frac{\pi}{2}\left(Q_{1} Q_{5}-2 N^{2} J\right)\left(Q_{1} Q_{5}-N^{2} J\right)^{-3 / 2} \\
g_{Q_{1} J} & =-\frac{\pi}{2} N^{2} Q_{5}\left(Q_{1} Q_{5}-N^{2} J\right)^{-3 / 2} \\
g_{Q_{5} Q_{5}} & =\frac{\pi}{2} Q_{1}^{2}\left(Q_{1} Q_{5}-N^{2} J\right)^{-3 / 2} \\
g_{Q_{5} J} & =-\frac{\pi}{2} N^{2} Q_{1}\left(Q_{1} Q_{5}-N^{2} J\right)^{-3 / 2} \\
g_{J J} & =\frac{\pi}{2} N^{4}\left(Q_{1} Q_{5}-N^{2} J\right)^{-3 / 2}
\end{aligned}
$$

We may thus stress out that the state-space geometry, materializing from the Bekenstein-Hawking entropy of the $D_{1}-D_{5}$ configurations, admits remarkably simple expressions in terms of physical charges and angular momentum. It may thence be expected that the plausible microscopic preliminaries would be suggested via the Cardy formula, or the associated general Hardy-Ramanujan formula. As enumerated in the earlier sections, we may again appreciate, for all non-zero values of the brane charges $Q_{1}, Q_{5}, J$ and angular momentum $J$, that the principal components of present state-space metric tensor satisfy

$$
\begin{aligned}
g_{Q_{1} Q_{1}} & >0 \\
g_{Q_{5} Q_{5}} & >0 \\
g_{J J} & >0
\end{aligned}
$$

Physically, the principal components of the state-space metric tensor signify heat capacities, or the relevant compressibilities, whose positivity connote that the underlying system is in locally stable equilibrium configurations of the $D_{1}$ and $D_{5}$-brane microstates. Furthermore, it may easily be observed that the ratio of diagonal components varies as the inverse square of the invariant parameters which vary under the Gaussian fluctuations, whereas the ratios involving off diagonal components vary only inversely in the chosen parameters. This suggests that the diagonal components weaken faster and relatively more quickly come into an equilibrium, than the off diagonal components, which remain comparable for the longer domain of invariant parameters defining the $D_{1}-D_{5}$ configurations. In particular, we may easily substantiate, for the distinct $i, j \in\{1,5\}$, that the relative pair correlation functions satisfy the following simple relations:

$$
\begin{aligned}
\frac{g_{Q_{i} Q_{i}}}{g_{Q_{j} Q_{j}}} & =\left(\frac{Q_{j}}{Q_{i}}\right)^{2} \\
\frac{g_{Q_{i} Q_{i}}}{g_{J} J} & =\left(\frac{Q_{j}}{N^{2}}\right)^{2} \\
\frac{g_{Q_{i} J}}{g_{Q_{j} J}} & =\frac{Q_{j}}{Q_{i}} \\
\frac{g_{Q_{i} Q_{i}}}{g_{Q_{i} J}} & =-\frac{Q_{j}}{N^{2}}
\end{aligned}
$$

Moreover, it is very instructive to note that the behavior of brane-brane statistical pair correlation, defined as $g_{Q_{1} Q_{5}}$, is asymmetric, in contrast to the other existing correlations. In fact, one may understand it by arguing that the brane-brane interaction imparts more energy than either the interaction corresponding to the self-interactions or that of the rotations. In particular, our analysis proclaims that the relative pair correlation between the $D_{1}-D_{5}$ branes, with respect to rotation-rotation correlation turns out to be

$$
\frac{g_{Q_{1} Q_{5}}}{g_{J J}}=-\frac{1}{N^{4}}\left(Q_{1} Q_{5}-2 N^{2} J\right)
$$

This implies that the relative brane-brane statistical pair correlation function vanishes exactly at the half value of the angular momentum, compared to that of the vanishing entropy condition. Furthermore, it may easily be viewed, from the non-vanishing central charge, that the brane-brane pair correlation is stable, only if the underlying angular momentum satisfies a lower bound $|J|<\frac{Q_{1} Q_{5}}{2 N^{2}}$. In order to entail definite global properties of the concerned configurations, one may now be required to determine stabilities along each direction and along each plane, as well as on the entire intrinsic state-space manifold. 
It is worth to note that the appraisal of exhaustive state-space stability demands that all the associated principal minors must be positive definite, as the positivity of principal components of metric tensor is defined only in the local neighborhood of a certain chosen co-ordinate chart of the state-space manifold $\left(M_{3}, g\right)$. It is nevertheless not difficult to compute the principal minor of the associated Hessian matrix of the entropy, concerned with the rotating two charged black holes. It is rather easy to explain the physical picture of the solution set, and in fact, after some simplifications, one discovers that the planar stability criteria on the two dimensional surfaces of the state-space manifold may simply be rendered to be

$$
p_{2}=\pi^{2} N^{2} J\left(Q_{1} Q_{5}-N^{2} J\right)^{-2}
$$

In the view-points of the simplest $D_{1}-D_{5}$ solutions, it tuns out that the local stability on the entire equilibrium phase-space configurations may clearly be determined by computing the determinant of the underlying statespace metric tensor. As in the previous examples, it is easy to observe that the state-space metric tensor is a non-degenerate, and everywhere regular, function of the brane charges and angular momentum. In particular, we may conveniently ascertain that the determinant of the metric tensor obtains a simple form

$$
g\left(Q_{1}, Q_{5}, J\right)=-\frac{1}{2} \pi^{3} N^{4}\left(Q_{1} Q_{5}-N^{2} J\right)^{-5 / 2}
$$

Here, we deduce that the determinant of the metric tensor does not take a well-defined positive definite form, and thus there is no positive definite globally well-defined volume form on the state-space manifold $\left(M_{3}, g\right)$, for the concerned systems. Moreover, the non-zero state-space determinant $g\left(Q_{1}, Q_{5}, J\right)$, thus constructed, indicates that the extremal $D_{1}-D_{5^{-}} J$ systems may decay into certain degenerate vacuum state configurations, procuring the same corresponding microscopic entropy. In turn, we may thus notify that the $D_{1^{-}} D_{5^{-}} J$ black holes, when considered as a bound state of the $D_{1}-D_{5}$-brane microstates, do not correspond to an intrinsically stable statistical configuration. It is worth to notice that the conclusions thus drawn remain independent of the microscopic type-II string description, or the heterotic string description.

Furthermore, we may exhibit that the nature of the statistical interactions and the other global properties of the $D_{1}-D_{5^{-}} J$ configurations are indeed not really perplexing to anatomize. In this regard, one only needs to compute certain global invariants of the state-space manifold $\left(M_{3}, g\right)$, which can thus be determined in terms of the parameters of underlying brane configurations. Here, we may work in the large charge limit, in which the asymptotic expansion of the entropy of the two charge rotating $D_{1}-D_{5}$ system is valid. In particular, we notice that the state-space scalar curvature may be appraised by the expression

$$
R\left(Q_{1}, Q_{5}, J\right)=-\frac{5}{4 \pi}\left(Q_{1} Q_{5}-N^{2} J\right)^{-1 / 2}
$$

It is worth to mention that the scalar curvature thus determined never vanishes, for all physically acceptable value of the brane charges and angular momentum, and thus it is an everywhere non-vanishing function on the state-space manifold $\left(M_{3}, g\right)$. The negative sign of the state-space scalar curvature signifies that the underlying statistical system is effectively an attractive configuration, and thus it is less stable than the arbitrary positively curved equilibrium intrinsic configurations. We may also notice, in the present case, that the scalar curvature $R\left(Q_{1}, Q_{5}, J\right)$ is a regular function of the $\left\{Q_{1}, Q_{5}, J\right\}$ coordinatizing the underlying statespace configuration. Thus, the underlying state-space configuration remains a non-degenerate, everywhere finitely curved, intrinsic Riemannian manifold, which is free from quantum instabilities and vacuum phase transitions. This makes sure, for all non-zero brane degeneracies, that the $D_{1}-D_{5}$ systems always correspond to certain weakly interacting statistical configurations. It follows again that both the intriguing curves of constant entropy $k_{1}$ and constant state-space scalar curvature $k_{2}$ take the same form

$$
Q_{1} Q_{5}=N^{2} J+c_{i}
$$

This configuration thus defines an existence of veritable bounds on the collection of degenerate correlated $D_{1}-D_{5}$ microstates, which is indeed not surprising for the two charged rotating black holes. The concerned bounds may certainly be anticipated from the degeneracy of CFT microstates and from the derived statistical correlations, which both depend on the corresponding phase-space configuration of the $D_{1}-D_{5}$ solutions. Such bounds, associated with the degeneracy of the microstates, may as well be obtained from the central charge of an underlying four dimensional holographic dual conformal field theory. Furthermore, it is easy to notice in the present case that the constants $c_{i}$ may respectively be defined as

$$
\begin{aligned}
c_{1} & :=\frac{k_{1}^{2}}{4 \pi^{2}} \text { for constant entropy } \\
c_{2} & :=\frac{25}{16 \pi^{2} k_{2}^{2}} \text { for constant scalar curvature }
\end{aligned}
$$


Moreover, we find an intriguing relation of the conclusions obtained from our state-space geometry with the Mathur's fuzzball proposal of constructing microstates of the two charged rotating black holes. As exercised in the connection with Mathur's fuzzball proposal, the typical microstates of an extremal black hole cannot acquire a space-time singularity [37. Similarly, we may deduce from the present consideration that even an intrinsic Riemannian manifold of the equilibrium microstates continues to remain non-singular. In other words, the regularity of the state-space geometric curvature invariant exposes that the intrinsic Riemannian geometry constructed out of the parameters of rotating two charge $D_{1}-D_{5}$ configurations as extrema of the entropy, also remains regular. Consequently, the absence of divergences in the state-space scalar curvature implies that the underlying statistical systems of the two charge rotating black holes are thermodynamically stable under the Gaussian fluctuations, and thus there are no vacuum phase transitions in the counterpoised microscopic configurations.

Very similar conclusions are also trivially accessible for the case of a more complicated $U(1)^{n}$ supergravity theory. The general features of these solutions are rather involved, however we find worth mentioning that they inspire some considerably interesting state-space characteristics. In the next subsection, we shall generalize the above outlined state-space configuration of the two charged $D_{1}-D_{5}$ solutions to the case of three charged $D_{1}-D_{5}-P$ configurations. In particular, we may stimulate the present investigation for the spherical horizon topology BMPV black holes. In order to adequately analyze these solutions, we shall put our emphasis on the standard $D_{1}-D_{5}-P$ frames and their dual $M$-theory backgrounds, to show that the state-space consideration pursued in this subsection germinates an analogous bound on the admissible angular momentum, and that similar definite conclusions arise for the three charge rotating configurations.

\subsection{BMPV Black Holes}

The present subsection examines the state-space configuration of the spherical topology black holes, and experiments to extend our intrinsic geometric assessments, for the known supersymmetric black hole solutions, in five-dimensional supergravity with a constant horizon topology, see for details [107]. For the purpose of critical ratifications, we shall focus our attention on the BMPV black holes, which may be obtained as the limiting case of supersymmetric black rings, when one takes zero-limit of the ring radius, with the space-time coordinates and all other parameters of the solution held fixed. The solution under interest may thus be ascertained by four parameters, viz., $Q_{i}$ and $J$, where the former retain their interpretation as conserved $M_{2}$-brane charges, while the latter determines the angular momentum: $J:=J_{\phi}=J_{\psi}$ of the concerned BMPV black hole configurations. It is known that the BMPV solutions are much more symmetrical than the supersymmetric black rings, as they have non-trivial isometry group: $\mathbf{R} \times U(1) \times S U(2)$, see for details [108. Here, the upshot is that we intend to discover the possible definitive conclusions emerging from the event horizon, which has a topology of $S^{3}$, and sits at the origin of the space-time solution.

In order to explicate the state-space geometry of spherical BMPV black holes in the $D_{1}-D_{5}-P$ frame, we may consider a five dimensional string in the maximally twisted sector with a linear momentum, which is among one of the three conserved charges carried by both the bosonic and fermionic excitations along the effective string. In particular, the possible fermionic excitations may also carry a polarization in the transverse directions, which emerges due to the non-vanishing R-charges appearing in the associated UVCFT. Thus, for a given number of oscillators polarized in the same direction with a self-dual angular momentum, the projection of oscillators onto the ascribed polarization, which restricts the phase-space configurations, acquiesces our purpose for analyzing the nature of underlying statistical interactions.

Here, one may infer, via the Cardy formula, that the entropy of the supersymmetric BMPV rotating black holes may easily be transcribed as a function of brane numbers, the self-dual angular momentum, and it does not entangle with the Kaluza-Klein excitations or the $M_{5}$-brane charges, see for instance [107]. The BMPV balck holes thus arise as an intriguing special case of the generic $M$-theory solutions, when the dipole charges become redundant. In particular, one discovers that the horizon of these solutions topologically reduces to the three sphere $S^{3}$. We thus observe that the analysis of state-space geometry would present a detailed account of the statistical correlations and other associated physical properties possessed by the spherical horizon BMPV black hole configurations.

For the purpose of our essential analysis, let us consider three independent asymptotic brane charges $\left\{Q_{1}, Q_{2}, Q_{3}\right\}$ and an angular momentum $J$ describing the rotating BMPV configurations. As just mentioned, the entropy of the spherical topology BMPV black holes, arising from the area of the event horizon, may be disclosed in terms of the brane charges and an angular momentum 107. In particular, one finds that the entropy of the BMPV black holes divulges a simple expression

$$
S\left(Q_{1}, Q_{2}, Q_{3}, J\right)=2 \pi \sqrt{\pi^{2} Q_{1} Q_{2} Q_{3}-16 G^{2} J^{2}}
$$

As proclaimed in the previous subsections, we notice, in this case also, that the state-space geometry describing the nature of equilibrium brane microstates may be constructed out of the three charges and angular momentum of the rotating spherical topology BMPV black holes. As invoked earlier, the covariant 
metric tensor may immediately be computed from the negative Hessian matrix of the concerned entropy, resulting from the underlying horizon area of the configration. Thus, the asymptotic invariant charges and angular momentum: $\left\{Q_{1}, Q_{2}, Q_{3}, J\right\}$ form the coordinate charts for the state-space manifold of our interest. Thus, with respect to these parameters, we may describe the typical intrinsic geometric features of the BMPV black holes. We thence notice that the components of the covariant metric tensor may easily be presented to be

$$
\begin{aligned}
g_{Q_{1} Q_{1}} & =\frac{1}{2} \pi^{5} Q_{2}^{2} Q_{3}^{2}\left(\pi^{2} Q_{1} Q_{2} Q_{3}-16 G^{2} J^{2}\right)^{-3 / 2} \\
g_{Q_{1} Q_{2}} & =-\frac{1}{2} \pi^{3} Q_{3}\left(\pi^{2} Q_{1} Q_{2} Q_{3}-32 G^{2} J^{2}\right)\left(\pi^{2} Q_{1} Q_{2} Q_{3}-16 G^{2} J^{2}\right)^{-3 / 2} \\
g_{Q_{1} Q_{3}} & =-\frac{1}{2} \pi^{3} Q_{2}\left(\pi^{2} Q_{1} Q_{2} Q_{3}-32 G^{2} J^{2}\right)\left(\pi^{2} Q_{1} Q_{2} Q_{3}-16 G^{2} J^{2}\right)^{-3 / 2} \\
g_{Q_{1} J} & =-16 \pi^{3} G^{2} J Q_{2} Q_{3}\left(\pi^{2} Q_{1} Q_{2} Q_{3}-16 G^{2} J^{2}\right)^{-3 / 2} \\
g_{Q_{2} Q_{2}} & =\frac{1}{2} \pi^{5} Q_{1}^{2} Q_{3}^{2}\left(\pi^{2} Q_{1} Q_{2} Q_{3}-16 G^{2} J^{2}\right)^{-3 / 2} \\
g_{Q_{2} Q_{3}} & =-\frac{1}{2} \pi^{3} Q_{1}\left(\pi^{2} Q_{1} Q_{2} Q_{3}-32 G^{2} J^{2}\right)\left(\pi^{2} Q_{1} Q_{2} Q_{3}-16 G^{2} J^{2}\right)^{-3 / 2} \\
g_{Q_{2} J} & =-16 \pi^{3} G^{2} J Q_{1} Q_{3}\left(\pi^{2} Q_{1} Q_{2} Q_{3}-16 G^{2} J^{2}\right)^{-3 / 2} \\
g_{Q_{3} Q_{3}} & =\frac{1}{2} \pi^{5} Q_{1}^{2} Q_{2}^{2}\left(\pi^{2} Q_{1} Q_{2} Q_{3}-16 G^{2} J^{2}\right)^{-3 / 2} \\
g_{Q_{3} J} & =-16 \pi^{3} G^{2} J Q_{1} Q_{2}\left(\pi^{2} Q_{1} Q_{2} Q_{3}-16 G^{2} J^{2}\right)^{-3 / 2} \\
g_{J J} & =32 \pi^{3} G^{2} Q_{1} Q_{2} Q_{3}\left(\pi^{2} Q_{1} Q_{2} Q_{3}-16 G^{2} J^{2}\right)^{-3 / 2}
\end{aligned}
$$

Nevertheless, it is handy to observe that the statistical pair correlations, thus ascertained, may in turn be accounted by a simple microscopic description, expressed in terms of the brane charges and angular momentum, connoting an ensemble of microstates for the supersymmetric BMPV black holes. Furthermore, it is evident that the principal components of the statistical pair correlations are positive definite, for all allowed values of the asymptotic invariant parameters of the black holes. In particular, we may easily observe that the concerned state-space metric constraints are

$$
\begin{aligned}
g_{Q_{1} Q_{1}} & >0 \forall\left(Q_{1}, Q_{2}, Q_{3}, J\right) \\
g_{Q_{2} Q_{2}} & >0 \forall\left(Q_{1}, Q_{2}, Q_{3}, J\right) \\
g_{Q_{3} Q_{3}} & >0 \forall\left(Q_{1}, Q_{2}, Q_{3}, J\right) \\
g_{J} & >0 \forall \text { admisible }\left(Q_{1}, Q_{2}, Q_{3}, J\right) \mid Q_{i} \neq 0
\end{aligned}
$$

Essentially, the principal components of the state-space metric tensor $\left\{g_{Q_{i} Q_{i}}, g_{J J} \mid i=1,2,3\right\}$ signify a set of definite heat capacities (or the related compressibilities), whose positivity apprises that the BMPV black holes comply an underlying locally equilibrium statistical configuration. It is intriguing to note that the positivity of $g_{J J}$ requires that none of the brane charges of the associated $D_{1}-D_{5}-P$ CFT (or $M_{2}$-brane charges in the dual description) should be zero. This is clearly perceptible, because of the fact that the brane configuration becomes unphysical.

It follows from the above expressions that the ratio of the principal components of statistical pair correlations varies as an inverse square of the asymptotic charges; in contrast, that of the off diagonal correlations modulates only inversely. Interestingly, we may easily visualize, for the distinct $i, j, k \in\{1,2,3\}$, that the statistical pair correlations, thus described, are consisting of the following scaling properties:

$$
\begin{aligned}
\frac{g_{Q_{i} Q_{i}}}{g_{Q_{j} Q_{j}}} & =\left(\frac{Q_{j}}{Q_{i}}\right)^{2} \\
\frac{g_{Q_{i} Q_{i}}}{g_{J} J} & =\frac{\pi^{2}}{64 G^{2}}\left(\frac{Q_{j} Q_{k}}{Q_{i}}\right) \\
\frac{g_{Q_{i} J}}{g_{Q_{j} J}} & =\frac{Q_{j}}{Q_{i}} \\
\frac{g_{Q_{i} Q_{i}}}{g_{Q_{i} J}} & =-\frac{\pi^{2}}{32 G^{2}}\left(\frac{Q_{j} Q_{k}}{J}\right) \\
\frac{g_{Q_{i} Q_{j}}}{g_{Q_{k} J}} & =\frac{1}{32 G^{2}}\left(\frac{Q_{k}}{J Q_{i} Q_{j}}\right)\left(\pi^{2} Q_{1} Q_{2} Q_{3}-32 G^{2} J^{2}\right) \\
\frac{g_{Q_{i} Q_{j}}}{g_{Q_{i, j} Q_{k}}} & =\frac{Q_{k}}{Q_{j, i}}
\end{aligned}
$$


As noticed in the previous configurations, it is not difficult to analyze the local stability for the BMPV black holes, as well. In particular, one may easily determine the principal minors associated with the state-space metric tensor. Thus, we may demand that all the principal minors must be positive definite. In this case, we may adroitly compute the principal minors from the Hessian matrix of the associated entropy concerning the three charge rotating BMPV black holes. In fact, after some simple manipulations we discover that the local stability criteria on the three dimensional hyper-surfaces and the two dimensional surfaces of the underlying state-space manifold may respectively be given by the following relations:

$$
\begin{aligned}
& p_{3}=8 \pi^{9} Q_{1} Q_{2} Q_{3}\left(-\pi^{2} Q_{1} Q_{2} Q_{3}+64 G^{2} J^{2}\right)\left(\pi^{2} Q_{1} Q_{2} Q_{3}-16 G^{2} J^{2}\right)^{-5 / 2} \\
& p_{2}=16 \pi^{6}\left(Q_{3}^{2} G^{2} J^{2}\right)\left(\pi^{2} Q_{1} Q_{2} Q_{3}-16 G^{2} J^{2}\right)^{-2}
\end{aligned}
$$

For all physically admitted values of invariant asymptotic charges of the BMPV black holes, we may thus easily ascertain that the minor constraint, viz., $p_{2}\left(Q_{i}, J\right)>0$ inhibits the domain of assigned angular momentum, that must be a positive definite real number, while the constraint $p_{3}\left(Q_{i}, J\right)>0$ imposes that the angular momentum must respectively be greater than $J=\frac{\pi}{8 G}\left(Q_{1} Q_{2} Q_{3}\right)^{1 / 2}$. In particular, these constraints enable us to investigate the nature of the state-space geometry of the BMPV black holes. We may thus observe that the presence of planar and hyper-planar instabilities is established for the spinning BMPV black holes, which together demand for a restriction on the allowed value of the angular momentum.

Furthermore, we find that it is easy to determine the complete local stability on the full phase-space configuration, which may be acclaimed by computing the determinant of the state-space metric tensor. Nevertheless, it is not difficult to enumerate a compact formula for the determinant of the metric tensor. For the different possible values of the charges, viz., $\vec{Q}:=\left(Q_{1}, Q_{2}, Q_{3}\right)$ and the angular momentum $J$, it may apparently be discovered from the present intrinsic geometric analysis, that the following expression holds for the determinant of the metric tensor:

$$
g(\vec{Q}, J)=-16 \pi^{12} G^{2} Q_{1}^{2} Q_{2}^{2} Q_{3}^{2}\left(\pi^{2} Q_{1} Q_{2} Q_{3}-16 G^{2} J^{2}\right)^{-3}
$$

The determinant of the basic metric tensor is a small, non-zero quantity, in the large charge limit, in which one acquires a non-vanishing central charge of the corresponding $D_{1}-D_{5}-P$ CFT, or the associated worldvolume notion of the dual $M$-theory. Our analysis further discovers that there exists a non-degenerate thermodynamic configuration with the extremal contributions. However, it is worth to note that the determinant of the metric tensor does not take a positive definite form, which thus shows that there is no positive definite volume form on the concerned state-space manifold $\left(M_{4}, g\right)$ of the spinning BMPV black holes. This is also intelligible from the fact that the responsible equilibrium entropy tends to its maximum value, while the same may not remain valid on the chosen planes or hyper-planes of the entire state-space manifold. It may in turn be envisaged, in either the $D_{1}-D_{5}-P$ description or $M$-theory description, that the BMPV black holes do not correspond to an intrinsically stable statistical configuration. Thus, it is very probable that the underlying ensemble of microstates may smoothly move into more stable brane configurations.

Finally, in order to elucidate the universal nature of statistical interactions and the other properties concerning the rotating BMPV black holes, one needs to determine certain global geometric invariants on the state-space manifold $\left(M_{4}, g\right)$. Here, we note that such a simplest invariant may be achieved by the state-space scalar curvature, which may indeed be computed in the straightforward fashion by applying the formerly explained method of intrinsic geometry. It turns out that the state-space configuration of the BMPV black holes is entirely simple, and in particular, the explicit expression for the scalar curvature may be given to be

$$
R(\vec{Q}, J)=\frac{3}{4 \pi^{3} Q_{1} Q_{2} Q_{3}}\left(\pi^{2} Q_{1} Q_{2} Q_{3}-64 G^{2} J^{2}\right)\left(\pi^{2} Q_{1} Q_{2} Q_{3}-16 G^{2} J^{2}\right)^{-1 / 2}
$$

It may therefore be perceived, for the allowed value of conserved charges, that the state-space scalar curvature remains a negative definite quantity. This makes perfect physical sense, i.e. that the underlying system is effectively an attractive configuration. Here, the negative sign of the scalar curvature further signifies that it is a less stable black hole system, than the definite statistical configurations possessing certain positive statespace scalar curvature. Moreover, it is stimulating to expose that the scalar curvature thus demonstrated is an everywhere regular function on the state-space manifold, and in particular, it never diverges for the physically ratified values of $D_{1}-D_{5}-P$ or dual $M_{2}$-brane charges and the self angular momentum. It may thus be indicated that the rotating BMPV black holes have no phase transitions and the underlying statistical configuration is completely free from the critical phenomenon, in all tangibly admissible domain of brane charges and angular momentum, describing the five dimensional minimal supergravity BMPV solutions. 
In general, it may possibly be pronounced, for all non-zero brane charges, that the BMPV solutions render to be weakly interacting statistical configurations, whenever there exists a non-vanishing state-space scalar curvature. However, it turns out that the state-space scalar curvature vanishes for an absolute value of the angular momentum. In this specific case, one may deduce that the underlying configuration turns out to be a non-interacting statistical system and, thereafter, it changes the nature of the interactions. In particular, we may simply celebrate that the changes in the nature of statistical interactions happen precisely at the absolute value of angular momentum ascribed by

$$
|J|=\frac{\pi}{8 G} \sqrt{Q_{1} Q_{2} Q_{3}}
$$

Furthermore, it is worth to mention that the state-space quantities thus described entail various intriguing generic intrinsic geometric properties, and in particular we may easily notice, for the non-vanishing set of charges, that the determinant of the metric tensor remains non-zero, while the scalar curvature clearly vanishes at the half value of the angular momentum, in contrast to the zero entropy BMPV black holes. It may further be envisaged, for the other possible supergravity solutions in five dimensions, that they should indicate an interacting underlying statistical configuration, in accordance with the minimal supergravity BMPV configurations. In these cases, it is interesting to note that the multifaceted contribution of $M_{5^{-}}$ branes may be non-vanishing, and there would naturally be a higher number of charges. Thus, the underlying state-space manifold would relatively be a higher dimensional intrinsic Riemannian manifold, than the one analyzed for the restricted spherical horizon BMPV black holes.

We may however discover, for equal $M_{2}$ brane charges, viz., $Q_{i}=Q, i=1,2,3$, that the determinant of the state-space metric tensor and the associated scalar curvature reduce to their corresponding easily determinable non-vanishing values. It is worth mentioning that the indispensable contents of the statespace geometry, thus elucidated, entangle with the notion of planar and hyper-planar stabilities. These constraints may as well arise from the degeneracy of an ensemble of brane microstates constituting an equilibrium statistical configuration. With a definite entropy of the spinning BMPV black holes, we may in fact see that the constant entropy curve, for a given entropy $S_{0}$, take the form

$$
Q_{1} Q_{2} Q_{3}-\alpha J^{2}=\beta
$$

Here, it is obvious that the real constant $\alpha, \beta$ may easily be defined to be $\alpha:=16 G^{2} / \pi^{2}$ and $\beta:=S_{0}^{2} / 4 \pi^{4}$. Specifically, it is important to emphasize that the state-space scalar curvature, as a function of the parameters, entails the very possible existence of the phase-transitions, if any, underlying the spherical horizon BMPV configurations. In particular, we may observe, for some given scalar curvature $k$, that the constant scalar curvature curve turns out to be a simple curve which may rather be given as

$$
9\left(Q_{1} Q_{2} Q_{3}-4 \alpha J^{2}\right)^{2}=16 k^{2} \pi^{4} Q_{1}^{2} Q_{2}^{2} Q_{3}^{2}\left(Q_{1} Q_{2} Q_{3}-\alpha J^{2}\right)
$$

It is worth to mention that there exists a remarkable aspect of the supergravity solutions, that is the way in which they capture highly non-trivial constraints between the parameters that arise in their microscopic descriptions. In this concern, it is known, for spherical horizon BMPV black holes, that the condition that the space-time solutions be free from the causal pathologies, yields the correct upper bounds on the angular momentum required by the microscopic $D_{1}-D_{5}-P$ CFT or worldvolume theory. Such supergravity constraints do also arise from the state-space configuration, which may alternatively be apprised from the requirement that the solutions of interest admit an intriguing thermal deformation.

Nevertheless, we may assert that our state-space geometry determines certain intricate physical properties of the statistical pair correlation functions and correlation volume, which reveal the nature of associated parameters prescribing an ensemble of microstates of the dual CFT living on the boundary of chosen black brane configurations. Furthermore, we expect to consider it for a class of general higher dimensional black brane configurations, where the propositions of the ordinary state-space geometric computations might not be very feasible with multiple parameters. However, in such diverse situations one may possibly exhibit certain microscopic duality acquisitions, with an appropriate comprehension of the required parameters defining the state-space coordinate transformations on the concerned configurations.

In particular, our geometric concerns thus appraised may additionally be elucidated by the parameters uprising in both the string theory or associated $M$-theory descriptions and their gravity duals, from the very possible applications of an appropriate AdS/CFT correspondence. This clearly demonstrates that the space of physically relevant $D_{1}-D_{5}-P$-solutions is far from being completely mapped out, in our simple equilibrium state-space examinations. We shall leave these issues for the future, for an appropriate explanation for their microscopic origins. Further investigations are thus clearly needed, to establish a firm connection between the state-space, worldvolume and supergravity constructions, for the two and three-charge configurations studied in this paper. 


\section{Discussion of the Results}

In this Section we summarize each of the examples of geometries considered in the paper, from the viewpoint of the corresponding outlook and future investigations.

\subsection{Myers-Perry Black Holes}

The intrinsic Riemannian geometry of the rotating black brane configurations entails an interesting feature and, in particular, we deduce that the neutral Myers-Perry black holes admit a non-degenerate, negatively curved state-space geometry. Furthermore, we observe that the scalar curvature, as a function of the mass and angular momentum, exhibits a non-vanishing and regular structure over the entire state-space manifold. This indicates that the underlying statistical configuration is attractive and accompanies no phase transitions, under the inclusion of leading order statistical correlations.

Nevertheless, we note that the macroscopic understanding of the statistical interactions emerging from the attractor fixed point analysis may be accomplished, by specifying the types of moduli space geometry, while the corresponding large charged microscopic properties may be appraised, with an appropriate consideration of the $D$-brane or dual $M$-brane configurations. It is worth to mention that the issues concerning the statespace geometry of the Myers-Perry and Kaluza-Klein solutions may together be categorized, in a unified perspective of vacuum $M$-theory black holes. Thus, we wish to furnish the possible generalizations and their interpretations in the next subsection.

\subsection{Kaluza-Klein Black Holes}

We find, in the case of extremal Kaluza-Klein black holes, that the microscopic picture of state-space interactions may be apprehended, in terms of the net number of the underlying $D_{0}$ and $D_{6}$ branes. In fact, this characterization may also be exhibited, either by the individual brane masses, or in terms of the five dimensional angular momenta describing the interested Kaluza-Klein solutions. This is simply due to the fact that, when taking the product with the flat $T^{6}$, one obtains a solution to $M$-theory, whose IIA reduction acquires $D_{0}$ and $D_{6}$ charges. Nevertheless, it is known that these black holes are not supersymmetric, even in the extremal limit, which eludes nothing more than the absence of supersymmetric bound states of the $D_{0}$ and $D_{6}$ branes, see for details 83 . However, there are non-supersymmetric quadratically stable $D_{0}$ $D_{6}$ bound states [82, whose parameters intimate to serve a certain basis towards the microscopic picture of state-space interactions. Our analysis thus anticipates a simple stringy description to the state-space interactions, which rely on an understanding of microscopic entropy formula of the extremal black hole solutions.

More importantly, one can extend this argument to several other $M$-theory black holes and, in particular, there exist certain charged, nonsupersymmetric, extremal black branes [109, for which our state-space descriptions may be envisaged to be significant. It turns out that the state-space manifolds, thus outlined, are well-defined on each hyper-plane, and everywhere finitely curved on the full intrinsic configurations. However, unlike this simple natural example, we are here interested in describing the state-space manifolds of those higher dimensional black holes, which correspond to the general vacuum solutions in $M$-theory. In fact, the entropy of the pure black holes under consideration can indeed be microscopically comprehended, in terms of the Horowitz correspondence principal [110]. Although such evaluations do not reproduce the precise coefficient of the entropy, but up to a constant of proportionality, one may easily ascertain the state-space quantities. Thus, it is immediate to spell out the nature of Gaussian fluctuations, for these configurations.

Furthermore, certain attempts can be made to describe the statistical interactions geometrically, by different means, concerning the earlier interests which try to capture an accurate statistical entropy for the asymptotically flat black holes, including the neutral Myers-Perry solutions, as well 85.111. This insinuates that our interpretations of the metric tensor and scalar curvature are in perfect concordance with the known statistical behavior of the associated $D_{0}$ and $D_{6}$ black holes. In conclusion, the state-space geometry thus accounted gives rise to a microscopic comprehension of the fluctuating brane charge Kaluza-Klein black holes in string theory or vacuum $M$-theory. Here, the brane charge quantizations and energy excitation condition turn out to be certain general co-ordinate transformation on the state-space manifold. Thus, the state-space estimates may as well be transcribed in terms of these parameters of the extremal black holes [88, 112.

In the sequel, we out-line exotic interesting issues concerning the state-space geometry and possible interpretations for the neutral and charged $M$-theory black holes, which illustrate some natural generalizations in the other stimulating duality frames. We observe that the state-space geometry of the Kaluza-Klein black holes is not only an interesting statistical configuration, but it also ascribes an illuminating understanding of the phase transition, if any, critical exponents and other physical quantities, in terms of the parameters, either associated with the microscopic $D$-brane/ $M$-brane configurations, or with the macroscopic supergravity attractor solutions. It is important to note that one can implicitly procure an idea of microscopic 
descriptions of pair correlations and correlation volume, divulged in the framework of our state-space manifold. Furthermore, the state-space quantities thus obtained are indeed independent of the Kaluza-Klein radius, and the physical nature thence endures to be equivalent to that of the extremal Myers-Perry black holes, arising in the limit when the limit of infinite radius has been taken.

This is a strong indication that it would be feasible to determine an intriguing microscopic counting argument for the statistical pair correlations and correlation volume, which they may respectively be associated with the covariant and invariant quantities of the captivated state-space manifold. Our arguments have, as well, been supported by the fact that the entropy of the $M$-theory black holes depends on the corresponding integer normalized net charges on the brane solutions, in a chosen duality frame, see for further details 88 113]. In order to comprehend the state-space geometry of the most general Kaluza-Klein black holes, we may concentrate our attention on various possible open questions and their interpretations. Some of the issues in this concern may be summarized as follows.

Firstly, one should note that we have only examined the case of extreme Kaluza-Klein black holes. However, in order to divulge the full Kaluza-Klein configurations, one needs to comprehensively consider the corresponding near-extremal or non-extremal solutions, and then one would be obligated to count the microstates. If it is possible to obtain the degeneracy formulae in terms of the invariant parameters, then one can certainly take account of the concerned intrinsic geometric quantities, and there by our state-space analysis would explore the possible nature of correlations in the vacuum $M$-theory equilibrium configurations. These are precisely the ingredients that have also emerged from recent developments in the study of finitesized black-hole microstates in the vacuum $M$-theory black holes [88. Our analysis would thus furnish a unified framework for conceptualizing the underlying statistical fluctuations and their limiting geometric models, which may explore the nature of the general possible black brane configurations.

Interestingly, we identify, in these cases of more general solutions, that the mass is only an additional parameter, which gets added in the list of state-space variables. Thus, it may easily be anticipated that the extremal state-space manifold gets intrinsically embedded into an extra higher dimensional intrinsic Riemannian manifold, which describes the entire Kaluza-Klein configurations. In particular, it turns out, in the present case, that the following embedding may be defined for the extremal Kaluza-Klein black holes:

$$
\left(M_{3}, g\right) \rightarrow\left(M_{4}, \tilde{g}\right)
$$

Here, it is worth to mention that the extremal state-space configuration may be examined as the BPS restriction to the full counting entropy, with an intrinsic metric tensor, $g:=\left.\tilde{g}\right|_{M=M_{0}}$. It should be understood in this expression that the restriction $M=M_{0}$ has been applied to the assigned entropy of the non-extremal (or near extremal) Kaluza-Klein solutions. It may thus be envisaged, from the perspective of our state-space geometry, that an extremal $M$-theory vacuum configuration, defined as an embedding $M_{3} \hookrightarrow M_{4}$, would respectively be an interacting and stable configuration on the hyper-spaces of concerned non-extremal statespace configurations. Moreover, it is possible that the full non-extremal Kaluza-Klein configurations might comprise certain instabilities as well, whose signature would be encoded in the invariant scalar curvature of the corresponding state-space geometry.

Secondly, one can assimilate the state-space quantities of Kaluza-Klein black holes, with globally asymptotically flat solutions, simply by taking the limiting case of a single $D_{6}$-brane in the corresponding counting problem. Although such constructions are not yet rigorously exposed in the $M$-theory, as they require at least four globally flat asymptotic solutions, nevertheless it seems that the restricted two dimensional statespace manifold may eventually investigate the peculiarities of extremal Kaluza-Klein configurations. This is because an assignment of the entropy may be justified to each brane intersections, for a certain large number of such solutions. A similar construction would also be admissible for the non-extremal Kaluza-Klein configurations, for a single $D_{6}$-brane. In particular, one finds that there exists a restricted three dimensional state-space manifold, whose metric tensor and scalar curvature might respectively provide the properties of the statistical pair correlation functions and correlation volume of the brane system, in the scenario of a large number of intersecting brane solutions.

Thirdly, it may be expected that one can construct some natural generalizations of the above state-space investigation on certain general Calabi-Yau spaces, if it is still possible to ascribe a counting argument to the corresponding microscopic degeneracy of the brane microstates. Here, we would like to envisage in the spirit of a well-known fact associated with the $T^{6}$ moduli, i.e. that the corresponding mirror symmetry, defined as T-duality on a $T^{3}$ might be perceived as a certain coordinate transformation on the concerned state-space manifold. It is however exciting to notice that the initial collection of $D_{0}$ and $D_{6}$-branes goes over the collection of $D_{3}$-branes under this symmetry. Thus, there may exist certain endurances for the very constructed state-space correlations, as well. It would indeed be fascinating to find a link between the microscopic configurations thus originating, in the case of generalized Calabi-Yau spaces, and the state-space signatures which have been explicitly considered. Finally, we would like to mention that some of the observed intrinsic geometric issues need to be carefully explored. In particular, we intend that the approach being given in this paper may favorably provide a key understanding of how the fluctuating microstate geometries, 
in the semi-classical description, present state-space correlations, in the regime of parameters where a black hole exists.

\subsection{Supersymmetric $A d S_{5}$ Black Holes}

The state-space geometry arising from the supersymmetric $A d S_{5}$ black holes exhibits an interesting set of scaling characteristics for the statistical correlations. It is worth mentioning that the normalization convention of the electric charges $Q_{i}$ depends on the way in which one embeds the underlying gauged supergravity into the higher dimensional string theory, or associated $M$-theory configurations 33. Furthermore, there exists a natural normalization for the $A d S_{5} \times S^{5}$ background, such that the conserved electric charges $Q_{i}$, in the supersymmetric approximation, correspond to the internal Kaluza-Klein momenta akin to the $U(1)^{3} \subset S O(6)$ isometry.

More specifically, the microscopic indications of our intrinsic geometric estimations give an impression, i.e. that one may potentially describe the basis notions, either in the framework of dual giant gravitons, or in the construction of integral wrapped brane solutions. Here, the brane charges or brane numbers play an important role into the manifestly covariant computation of correlations and critical phenomena, if any, in the underlying statistical configurations. This is due to the fact that basic electric charges or brane numbers are respectively associated, either with the internal isometries of the theory, or with the topological cycles of the compactifying internal manifold. In particular, the charges $Q_{i}$ are integral for the consideration of the $A d S_{5} \times S^{5}$ or $U(1)^{3}$, and thus the interested state-space quantities arising from the concerned Bekenstein-Hawking entropy exhibit a set of simple and exact expressions, in the entire regime of the permissible physical parameters. It is further instructive to notice that such a simple explanation of the state-space analysis may shed light on the corresponding fluctuations, comprising the microscopic degeneracy and fundamental counting problem. It is thence expected that there would probably exist a general Cardy or Hardy-Ramanujan formula, which procures an account for its microscopic models, whose equilibrium configurations may geometrically be exposed, in the limit of Gaussian approximations.

Furthermore, we discover that the associated two point off-diagonal pair correlation functions vary as the sum of the inverse of two other brane charges, while the diagonal pair correlations vary as the sum of the inverse square of two other charges. We establish that the brane-brane pair correlation functions satisfy a set of definite asymmetric relations, in comparison with the other possible pair correlation functions, and nonetheless vanish exactly at the half angular momentum of the vanishing condition of the corresponding entropy. Here, one achieves the scalar curvature signifying the correlation length, which indicates that the associated microscopic configurations are stable in all possible hyper planes. This also elucidates that the corresponding interacting statistical systems are attractive configurations, for all non-vanishing values of the entropy of concerned black holes. Similar facts may further be enquired for the case of the spinning black holes in the Minkowski space, as well as in the richer isometric solutions of $\mathcal{N}=2$ supergravity.

It appears interesting to explore the state-space geometry of the $\frac{1}{16}$-BPS objects of type-IIB string theory, in $A d S_{5} \times S^{5}$ configurations [33. In particular, we may investigate the statistical fluctuations, for the supersymmetric black holes in gauged supergravity, and point out that there exists simple expressions for the state-space correlations arising from the Bekinstein-Hawking entropy, in terms of physical charges and rotation. We may thus appraise, from the simplicity of encompassed characteristics, that our analysis suggests an existence of non singular correlations in the underlying microscopic configurations, whose basis significance may be defined in terms of the parameters of the BPS gravitons.

One of the intriguing features of our state-space covariant objects, associated with the BPS black brane solutions assimilated in this paper, is that they might be viewed as the correlators of the parameters of the $\frac{1}{16}$-BPS deformations of either the $\frac{1}{8}$-BPS giant gravitons of [114], or those of the $\frac{1}{8}$-BPS dual giant gravitons of 115 . It would thus be exhilarating to contemplate our parametric geometric quantities in these dual microscopic descriptions. These interests emerge because the preferred solutions seem to be incorporated into a single solution space of the $\frac{1}{16}$-BPS theory. More strongly, we find at this point that if the entropy, as a real embedding function of the invariant asymptotic charges, endures fixed, then nothing much happens, except that there would be an existence of certain general co-ordinate transformations on the corresponding state-space manifold, which might change completely one degenerate configuration into the others.

We stress that the state-space geometry of $A d S_{5}$ black holes admit simple expressions not only for the $U(1)^{3}$ supergravity or truncated $S O(6)$ gauged supergravity, but also for those obtained by the very $S^{5}$ reduction of type-IIB string theory. The only requirement for supergravity is that the scalars in the vector multiplet should reside on a symmetric space, which may therefore be accomplished by the $A d S_{5}$ supergravity with at least 16 supersymmetries [16]. Moreover, we wish to further analyze our state-space construction, for the $\mathcal{N}=2 A d S_{5}$ supergravity configurations, accomplished by compactifying $M$-theory on a suitable 6-manifold. In this concern, there exists an explicit example known as Maldacena-Nunez solution, see for details 117.

It would thus be interesting to consider the state-space manifolds of $A d S_{5}$ black holes in this background, 
carrying a certain number of electric charges accomplishing from the membrane wrapping an internal 2-cycle, as well as those coming from the internal momenta, that are in turn analogous to $S^{5}$ Cartans of the theory. Furthermore, it is known that the central charge factor $c$ in front of the angular momentum $J$ is proportional to $N^{3}$, where $N$ is the number of $M_{5}$ branes, on which the dual superconformal field theory lives. In this concern, we would like to account for the D-instanton contributions to the graviton scattering amplitudes, and thus the corresponding $S U(N)$ Yang-Mills instanton corrections into the state-space correlations, see for a definite consideration [90]. Therefore, it would not be formidable to analyze the coarse graining prelude of the state-space quantities, with or without the instanton corrections, and the other associated microscopic properties transpiring in the $A d S$-black hole solutions.

\section{$5.4 \quad D_{1}-D_{5}$ Configurations}

With the most studied BPS black brane configurations, we notice that the state-space manifolds associated with the $D_{1}-D_{5}$ solutions 3 acquire the two striking microscopic interpretations. In general, one arrives at the conclusion that an akin representation exists in terms of either the $D_{1}-D_{5}-P$ CFT [118], or the fourdimensional black hole CFT [?,120]. On one hand, the first may be accounted via the invariant parameters of the CFT microstates, the former should be thought to be coming out of the ground states of $D_{1}-D_{5}-P$ black branes, while the latter may be connoted in the same way, as that of the parameters of the solutions of 39, 121, describing the five-dimensional three-charge black holes and the possible dual solutions of [122, which divulge the ground states of four dimensional four-charge black holes. This shows that there exist many interesting independent vacua of underlying $D_{1}-D_{5}-P$ CFTs. Thus, it is beyond the present scope to explicitly establish a particular microscopic composition, which corresponds to coarse grained state-space geometries.

Based on the microscopic dispositions of 39118,121], we may envisage that our state-space constructions would correspond, as well, to multiple brane configurations being dual to the CFT states, with longer effective strings than the solutions that come from only single brane. It may thus be pointed out that an effective string picture stimulates an interesting notion for the state-space pair correlation functions of well established $D_{1}-D_{5}$ black hole configurations. Moreover, our intrinsic Riemannian geometric analysis indicates that the maximum correlation length, emerging from the scalar curvature, involves the largest number of bubbles, which should correspond to the CFT states with the longest tangible effective strings. Nevertheless, it may be argued that the underlying statistical configurations of bubbled black branes are free from the instabilities and/ or vacuum phase transitions.

These inspirations stem naturally from the piercing mechanism of state-space geometry to the regular $D_{1}-D_{5}-P$ system. Moreover, the underlying physics is closely related to that of other possible solutions containing the correct black hole/ string/ ring/ supertube vacuum configurations. As mentioned earlier, the BPS black branes have two microscopic interpretations: one in terms of the $D_{1}-D_{5}-P$ CFT [118], the other in terms of the four dimensional black hole CFT [118 120]. Thus, an investigation may as well be explored for these solutions. Hence, our construction may be disclosed, in terms of the parameters of the $D_{1}-D_{5}-P$ system, and those of the four dimensional black hole CFT, which describes the BPS black branes. It may similarly be apprised that both the urged descriptions should play an important role in the determination of the correlated, interacting statistical basis, for the general $D_{1}-D_{5}-P$ solutions.

Finally, the results thus obtained insinuate a number of interesting consequences and intriguing relations for future exploration. One of the most influential possibility is to start from the microscopic $D_{1}-D_{5}-P$ description, and explore the zero-entropy limit of the concerned BPS black brane configurations. Here, the perceptible picture adduces that the most generic bound states with definite branes, which could be determined by a gas of positive and negative centers, with fluxes threading the many non-trivial two-cycles of the GH base, having no localized brane charges, are likewise possible to be elucidated [123 126. In turn, it is feasible to demonstrate, with several dual $M_{5}$-brane contributions as well, that there are no critical phenomenon on the non-degenerate state-space manifolds, thus spanned by the conserved parameters, in the vanishing entropy limit of the BPS solutions. Moreover, very similar conclusions are encouraged for the state-space geometric quantities defined for the other pertained definite string theory, or M-theory black holes.

\subsection{BMPV Black Holes}

The state-space of three charged $D_{1}-D_{5}-P$-solutions shows various interesting intrinsic Riemannian geometric properties, for the spherical horizon topology black holes. One of the intriguing features of the covariant structures, associated with the supersymmetric configurations, has been based on the fact that these solutions may uniquely be specified by their electric charges, and definite angular momenta, which in the limit of vanishing compactification radius lead to the rotating BMPV black holes of the present inquisitiveness. In fact, there are no other black hole solutions with a self dual angular momentum $J=J_{\psi}=J_{\phi}$. Wherefore, 
the conserved electric charges and the self-dual angular momentum of theory may always uniquely be chosen for the BMPV black holes, which in particular form a coordinate basis for the underlying intrinsic state-space manifold. Significantly, the geometric nature of the Gaussian fluctuation, thus celebrated, may properly be addressed, in terms of the invariant physical parameters of the chosen solution. In turn, it has been deduced that the state-space pair correlation functions admit simple scaling relations, while the associated correlation length of possible statistical configurations may as well be ascertained, in terms of the concerned invariant parameters of the theory.

On other hand, we have recently appraised various examples of a class of generic state-space manifolds, whose microscopic implications may be stimulated from the origins of coarse graining entropy of extremal and non-extremal black brane solutions. It may further be pointed out that the various statistical correlations, based over a large number of equilibrium microstates, are possible to be described in the framework of our state-space geometry, see for further detail [38. In a close connection with the exposed pursual, we pronounce that the ratio of various diagonal components of the BMPV state-space configuration scale as inverse square of the invariant asymptotic parameters varying under the Gaussian fluctuations, whereas the ratios involving the diagonal components transform only inversely in the chosen parameters. This implies that the diagonal components weaken faster, and they relatively more quickly attain an equilibrium statistical configuration, than the underlying off-diagonal components. In this case, we may further realize that the rotating BMPV black holes procure a weakly interacting statistical configuration, which has no phase transitions. The present analysis thus instructs that the microscopic configurations of BMPV black holes should be free from the critical phenomena.

Furthermore, it may be expected that alike statistical illuminations would exist in the physically admissible domain of brane charges and angular momenta, connoted by the miscellaneous multi-charged rotating configurations. Here, our interest is in particular on the very possible three-parameter supersymmetric black brane solutions [48, 127, 128 of minimal $D=5$ supergravities which, upon oxidation to the ten dimensions, describe black supertubes carrying a definite number of $D_{1}$-brane, $D_{5}$-brane and momentum charges. Nevertheless, the concerned systems abiding equal number of $D_{1}, D_{5}$ branes and Kaluza-Klein monopole with certain dipole moments, might further be investigated from the perspective of our intrinsic state-space geometry. It is tempting to annunciate that the familiar state-space geometry of these configurations may in general present many complexities, endorsed as the function of various promising invariant parameters. Such an interesting example may thus expound the case of black supertube solutions entangling the three charges, three dipole moments and the radius of ring. The concerned brane charges may as well be identified in the M-theory dual configurations, so that they ascribe the $M_{2}$ brane charges, while the dipole moments account for the $M_{5}$-charges.

Thus, the construction of our state-space geometry, enumerating most general $D_{1}-D_{5}-P$ systems, contains several previously divulged state-space geometries of 38 . Hitherto, it turns out, for the thrust of diversified analysis, that the interesting family of the possible solutions may be summarized as follows:

- Firstly, the state-space geometry thus developed would correspond to the one arising from the solution of 48, 127, 128, in the special case of three equal charges and three equal dipoles.

- Secondly, the state-space geometry may also expound to that of the two-charge supergravity configurations of 129, when one of the invariant charges and two dipoles vanish.

- Thirdly, one should analyze the other solutions, in the zero-radius limit, which reduce to the present solution having the four dimensional state-space geometry described in terms of the parameters of the supersymmetric spherical horizon BMPV black holes [107.

- Fourthly, one should compute the state-space geometric quantities of the $D_{1}-D_{5}-P$ solutions in the infinite-radius limit, which would test for the six-charge black strings of [130].

- Finally, one should examine the possible near-extremal and non-extremal cases of the full $D_{1}-D_{5}-P$ configurations. The concerned analysis would divulge a unified framework, for the intrinsic geometric understanding of possible statistical correlations. Thus, the nature of limiting microscopic models may be explored, for a class of general fluctuating black brane solutions.

\section{Conclusion and Outlook}

We studied state-space geometry of a class of higher dimensional rotating black holes, obtained after an adequate compactification of string theory and $M$-theory, and there by analyze the nature of underlying statistical configurations, by incorporating fluctuations into them. Mainly, the focus has been on the physical parameters labeling the black brane configurations, which divulge the microscopic and macroscopic acquisitions, and there by critically examinining the possible implications arising from the Gaussian fluctuations, exhibiting the symmetric character of state-space metric tensor. The behavior of statistical fluctuations has been explored from the perspective of an intrinsic Riemannian geometry, emerging from the negative 
Hessian of the coarse graining entropy defined over an ensemble of microstates. It has been demonstrated that the principal components of state-space metric tensor, which signify heat capacities or the associated compressibilities are generically positive definite. It is illuminating to notice that very similar state-space geometric conclusions are accomplished for both neutral and charged black hole solutions, being present in the vacuum $M$-theory.

We manifestly show that the relative diagonal components weaken relatively faster, and thus they more easily come into an equilibrium configuration, than the off diagonal components, which remain comparable for a longer domain of the parameters varying under the Gaussian fluctuations. In general, we observe that the brane-brane statistical pair correlation functions are asymmetrical in nature, in contrast to the other possible statistical pair correlation functions. This scaling property may naturally be expected from the fact that the brane-brane interactions impart more energy than either the self-interactions or the energy due to the rotations. This asymmetry generically intimates a lower bound on the angular momentum carried by the black holes. We discover that the state-space geometry thus described is non-degenerate and finitely curved for the neutral Myers-Perry black holes and charged Kaluza-Klein black holes. This exhibits that the vacuum $M$-theory black holes are a statistical system in a local equilibrium. The possible intrinsic geometric results obtained against the Myers-Perry solutions are quite illuminating. The present consideration takes into account the fact that the underlying microscopic configurations are consisting of a large number of brane-antibranes.

Furthermore, we deduce that the state-space invariant quantities thus acquired are negative, in general, which connotes that the underlying black hole systems are effectively in an attractive configuration. Importantly, it has been noted that the determined scalar curvatures never vanish, for a domain of the physically justifiable value of invariant charges and angular momentum, for the favored black hole solutions. This shows that these simple black holes correspond to an interacting statistical configuration, whose state-space scalar curvatures rapture out, to be an everywhere regular function of the composing parameters. For these natural systems, our results suggest that there exists an intriguing relationship between non-ideal interparticle (or more generally inter-brane) statistical interactions and thermodynamic phase transitions, which may in particular be established from the perspective of an intrinsic Riemannian manifold, or vice-versa. Our observations thus described are in turn consistent with the existing illustrations of the microscopic CFT data of the corresponding black brane configurations.

In this paper, we have contemplated that the state-space geometry, arising from the fluctuating higher dimensional rotating black brane configurations, may be exemplified by the string theory and $M$-theory. It is significant to notice that our state-space investigations may be based on the coarse graining phenomenon of the large number of degenerate CFT microstates describing an equilibrium statistical system, and thus they require no direct understanding of the microscopic black brane configurations. It has been discovered that the crucial ingredient, in analyzing the state-space manifold of the black brane configurations, only depends on the degeneracy of micro-states defining the counting entropy. Our conclusions are wherefore independent of the diverse microscopic properties of the elementary conformal field theories.

Such an interesting supergravity system includes the case of supersymmetric $A d S_{5}$ black holes and the accompanied $D_{1} D_{5}$ black brane configurations. For the $A d S_{5}$ configurations, we focus our attention on the symmetric spaces. There by it turns out that the statistical pair correlations comply truly captivating scaling properties. The state-space geometry, thus defined, turns out to be stable only in the plane and possible hyper-planes of the full state-space configuration, and the global parametric state-space is quadratically unstable. The state-space scalar curvatures indicate that the underlying microscopic theory is free from the possible phase transitions, and is a completely regular statistical configuration. These notions are more direct to pronounce for the most prevalent $D_{1}-D_{5}$ black brane systems, and here the supergravity conditions yield an intriguing stable and weakly interacting attractor solution. Here, it may be appreciated that the holographic corrections may efficiently be accounted for, by including the central charge term of the four dimensional boundary conformal field theory. The modifications of the state-space geometry are thus possible, due to these contributions. In particular, our conclusions show that the scalar curvatures, thus acquired, are only marginally sensitive to the plausible CFT modifications.

As a final exercise, we explored the case of BMPV black holes obtained from the toroidal $M$-theory compactification. Additionally, it is interesting to note that the dual $D_{1}-D_{5}-P$ description provides an illuminating framework, in order to examine the nature of statistical fluctuations into this system. The statespace geometry, thus intended, may indeed be shown to exhibit all the relevant properties and, in particular, one may analyze the geometric nature of the correlation functions and correlation volume of the concerned statistical configurations. Our computations present that the nature of statistical fluctuations admits an analogous form, actualized for the $A d S_{5}$ black hole configurations. It is worth to mention that the components of the state-space metric tensor, which comprise the two point statistical correlation functions, appear to be intertwined with the vacuum fluctuations of the boundary conformal field theory. This is because the required parameters of the black brane configurations describing the microstates of dual conformal field theory, which exists on the boundary, may be investigated by the AdS/CFT correspondence. 
The geometric formalism thus described entails with an ensemble of degenerate CFT ground states, which at an amusingly small constant positive temperature exhibits the statistical correlations into the basic equilibrium configurations. Furthermore, it is interesting to note that the Gaussian fluctuations about the equilibrium statistical configurations systematize the metric tensor on the intrinsic state-space manifolds. The behavior of brane-brane correlations in either cases turns out to be asymmetrical, in comparison with the other statistical pair correlation functions, and vanishes exactly at the half angular momentum, of the one materializing from the vanishing entropy condition. Our calculations display that the principal components of the state-space metric tensor are positive definite, while the non-identical components are not so, in general. Similarly, the surface and hyper-surface principal minors are positive definite functions of the invariant parameters, while for the full state-space determinants they turn out to be negative definite quantities.

The state-space geometries, thus exemplified for the higher dimensional rotating solutions, illustrate that the potential statistical configurations are stable, only if at least one of the parameter remains fixed. This is in perfect accordance with the well known fact that the only classical fluctuations having a thermal origin divulge with the classical probability distribution, which defines an intrinsic Riemannian metric tensor, over an ensemble of equilibrium microstates, and there by render to a weakly interacting and attractive statistical configuration. However, our state-space constructions, entangled with CFTs microstates, clearly celebrate that the degeneracy and the signature of the state-space geometry is indefinite, and the positive definiteness requirement is sensitive to the moduli space geometry and associated attractor solutions. Moreover, the absence of divergences in the scalar curvatures imply that the considered solutions are thermodynamically stable and there are no phase transitions in the underlying configurations.

The present investigation is thus motivated, as a prelude to the state-space geometry of an arbitrary higher dimensional configuration. Particularly, we have shown that the examples thus explored have an interesting set of state-space geometry, which describes the statistical fluctuations in the associated rotating black brane configurations. The details of black hole solutions, concerning the revealed intrinsic geometric enterprises, may further be observed. In general, we discover that the possible state-space geometry may be characterized by the following cases: (i) whenever there exists a positive definite determinant of the metric tensor, the underlying state-space configurations are everywhere well-defined, and the concerned black holes realize stable statistical configurations. (ii) whenever there exists a non-zero state-space scalar curvature, the underlying black hole configurations correspond to an interacting statistical system. Nevertheless, the sign of the state-space curvature characteristically plays an important role, in the determination of the nature of the interactions.

In this way, it is possible to expound, how the state-space pair correlation functions characterize the behavior of fluctuating statistical configurations over an ensemble of equilibrium microstates, defining the higher dimensional rotating black brane solutions. In such cases, it has indeed been interesting to determine, whether these correlations, as the function of all possible black brane charges, are positive definite and regular (or singular) functions on the enticed state-space manifold. Moreover, we have analyzed the stated black holes in the limit of vanishing state-space scalar curvatures, which makes perfectly clear the nature of limiting statistical configurations. Such limiting representations of the considered black hole solutions may as well be ascribed by the definite constant entropy and scalar curvature curves, which in turn algebraically describe the nature of corresponding state-space configuration. Furthermore, we could easily reveal, whether there exist certain critical points, or critical lines, and critical (hyper)-surfaces, in the fundamental configuration. In fact, if there exist any such critical qualifiers, then they all would exhaustively be distinguished by the divergence structure of the state-space scalar curvature.

The examples that we have accounted to analyze, for the rotating configurations, are neutral MyersPerry black holes, two charged Kaluza-Klein black holes, three charged $A d S_{5}$ black holes, two charged $D_{1} D_{5}$ configurations, and the associated spherical horizon BMPV black holes. Moreover, we find yet instructive to further explore the state-space geometry, for the general rotating black hole configurations emerging in the fundamental string theory and $M$-theory. In the previous section, we presented various possible results and explained the encompassed motivations for the specific cases of the extremal $M$-theory vacuum solutions, supersymmentric $D$-brane solutions comprising the two or higher charged rotating black brane configurations. In this respect, in the present investigation, the main line of thought has been to develop an intrinsic Riemannian geometric notion to the statistical fluctuations, which may be exercised between the most probable brane microstates of the string theory or $M$-theory.

We thence vindicate the coarse graining origins of the state-space geometry, arising from the negative Hessian matrix of the corresponding counting entropy, defined over an ensemble of brane microstates characterizing a chosen equilibrium statistical configuration. Furthermore, we have exhibited, from this perspective, that the state-space geometry remains non-degenerate and acquires a finite negative scalar curvature, for the exposed black holes at the attractor fixed points. Interestingly, the example of $D_{1}-D_{5} P$ solutions may further be interpreted by considering a large number of well separated Gibbon-Hawking charges, with a dissolving total Gibbon-Hawking charge. In particular, one may interrogate the scaling behavior of statistical pair 
correlation functions, describing the nature of various possible equilibrium microstates. In turn, our analysis reveals that these may easily be assessed, by the conserved parameters of the underlying microscopic CFT configurations. Finally, we are confronted with the general construction of our state-space geometry, and its potential role to describe the issue of interactions in associated higher dimensional rotating black brane configurations. In this concern, we have briefly addressed the result obtained for considered configurations, with numerous open developments for the future investigations.

\section{Appendix A}

We wish to explicitly give the involved intrinsic geometric quantities only for the case of two and three dimensional state-space manifolds. Furthermore, we may also exhibit that similar outlines hold for a class of general multi-parameter black brane configurations. For the two dimensional state-space geometry parametrically defined by the two invariant parameters, viz., an electric charge $Q$ and angular momentum $J$, we expose that the components of the covariant metric tensor may be given as

$$
\begin{aligned}
g_{Q Q} & =-\frac{\partial^{2} S}{\partial Q^{2}} \\
g_{Q J} & =-\frac{\partial^{2} S}{\partial Q \partial J} \\
g_{J J} & =-\frac{\partial^{2} S}{\partial J^{2}}
\end{aligned}
$$

We may therefore notice that the components of the state-space metric tensor are related to the statistical pair correlation functions, which may as well be defined in terms of the parameters describing the dual microscopic conformal field theory on the boundary. This is because the underlying metric tensor comprising Gaussian fluctuations of the entropy defines the state-space manifold for the rotating black brane configuration.

Moreover, the positivity of the state-space metric tensor imposes a stability condition on the Gaussian fluctuations of the underlying statistical configuration, which requires that the determinant and hyperdeterminant of the metric tensor must be positive definite. In fact, it is easy to express, in this simplest case, that the determinant of the metric tensor turns out to be

$$
\|g\|=S_{Q Q} S_{J J}-S_{Q J}^{2}
$$

In order to have a positive definite metric tensor on the two dimensional state-space geometry, one thus demands that the determinant of metric tensor must satisfy $\|g\|>0$, which in turn defines a positive definite volume form on the concerned state-space manifold. Furthermore, it is not difficult to calculate the Christoffel connection $\Gamma_{i j k}$, Riemann curvature tensor $R_{i j k l}$, Ricci tensor $R_{i j}$, and the scalar curvature $R$ for the two dimensional state-space intrinsic Reimannian manifold $\left(M_{2}, g\right)$. In particular, it turns out that the above two dimensional state-space scalar curvature appears as the inverse exponent of the inner product defining the pair correlation functions between two arbitrary equilibrium microstates characterizing the black brane statistical configuration. Explicitly, we find that the scalar curvature may be given by

$$
\begin{aligned}
R & =\frac{1}{2}\left(S_{Q Q} S_{J J}-S_{Q J}^{2}\right)^{-2}\left(S_{J J} S_{Q Q Q} S_{Q J J}+S_{Q J} S_{Q Q J} S_{Q J J}\right. \\
& \left.+S_{Q Q} S_{Q Q J} S_{J J J}-S_{Q J} S_{Q Q Q} S_{J J J}-S_{Q Q} S_{Q J J}^{2}-S_{J J} S_{Q Q J}^{2}\right)
\end{aligned}
$$

Notice that there exists an intriguing relation between the scalar curvature of the state-space intrinsic Riemannian geometry characterized by the parameters of the equilibrium microstates, with the correlation volume of the corresponding black brane phase-space configuration. It is worth to mention that the state-space scalar curvature in general signifies certain possible interactions in the underlying statistical configuration. Following our previous observations [38, it is in particular evident that the scalar curvature and corresponding Riemann curvature tensor of an arbitrary two dimensional intrinsic state-space manifold $\left(M_{2}(R), g\right)$ may be given by

$$
R=\frac{2}{\|g\|} R_{Q J Q J}
$$

Furthermore, we find that the general coordinate transformations on the state-space manifold, thus considered, may be expounded from the perspective of fundamental string duality relations associated with the invariant charges of the microscopic configurations. Indeed, we show, from the perspective of an intrinsic Riemannian geometry, that there exists an obvious mechanism on the black brane side, i.e. that it is possible to illuminate the statistical notion of associated correlations between the microstates of a brane solution or vice-versa. It turns out that the state-space constructions thus described clearly elucidate certain fundamental issues, such as statistical interactions and stability of the underlying brane configurations. In particular, 
it possible to provide an intrinsic geometric realization of the equilibrium statistical structures, which may be determined by the parameters of the CFT microstates describing the rotating black brane configurations.

Moreover, the state-space geometric structures thus disclosed may be expressed for the case of the higher dimensional intrinsic Riemannian manifolds, as well. In the case of the three dimensional state-space, we may easily define all the concerned quantities. Specifically, the components of the metric associated with the rotating black brane configurations carrying an electric charge $Q$, magnetic charge $P$, and an angular momentum $J$ are given by

$$
\begin{aligned}
g_{Q Q} & =-\frac{\partial^{2} S}{\partial Q^{2}} \\
g_{Q P} & =-\frac{\partial^{2} S}{\partial P \partial Q} \\
g_{Q J} & =-\frac{\partial^{2} S}{\partial Q \partial J} \\
g_{P P} & =-\frac{\partial^{2} S}{\partial P^{2}} \\
g_{P J} & =-\frac{\partial^{2} S}{\partial P \partial J} \\
g_{J J} & =-\frac{\partial^{2} S}{\partial J^{2}}
\end{aligned}
$$

\section{Appendix B}

In this appendix, we supply an explicit form of the most general state-space scalar curvature describing the family of two charged rotating black holes. Our analysis illustrates that the functional property of the specific scalar curvatures may exactly be exploited by the definite behavior of $f(Q, P, J)$. As we have accounted in section three, the various intriguing examples of the concerned solutions include Kaluza-Klein black holes in vacuum $M$-theory and the highly interesting $D_{1}-D_{5}$ systems. As mentioned in section 2 , we discover that the $f(Q, P, J)$ indicates the possible nature of general three parameter state-space configurations. In particular, one may notice, from the very definition of state-space metric tensor, that the relevant function $f(Q, P, J)$ could be computed to be

$$
f(Q, P, J):=\left(f_{1}-f_{2}\right)+2\left(f_{3}-f_{4}\right)+3\left(f_{5}-f_{6}\right)+2\left(2 f_{7}+3 f_{8}\right)
$$

where the $\left\{f_{i}\right\}$ are given by the following expressions

$$
\begin{aligned}
f_{1}= & \frac{\partial^{2} S}{\partial P^{2}} \frac{\partial^{2} S}{\partial Q^{2}} \frac{\partial^{3} S}{\partial Q^{2} \partial P} \frac{\partial^{3} S}{\partial P \partial J^{2}} \frac{\partial^{2} S}{\partial J^{2}}+\frac{\partial^{2} S}{\partial P^{2}} \frac{\partial^{3} S}{\partial Q^{3}} \frac{\partial^{2} S}{\partial Q \partial P} \frac{\partial^{2} S}{\partial P \partial J} \frac{\partial^{3} S}{\partial J^{3}} \\
& +\frac{\partial^{2} S}{\partial P^{2}} \frac{\partial^{3} S}{\partial P^{2} \partial J} \frac{\partial^{3} S}{\partial Q^{2} \partial J} \frac{\partial^{2} S}{\partial Q^{2}} \frac{\partial^{2} S}{\partial J^{2}}+\frac{\partial^{2} S}{\partial P^{2}} \frac{\partial^{3} S}{\partial Q \partial P^{2}} \frac{\partial^{3} S}{\partial Q \partial J^{2}} \frac{\partial^{2} S}{\partial Q^{2}} \frac{\partial^{2} S}{\partial J^{2}} \\
& +\frac{\partial^{2} S}{\partial P^{2}}\left(\frac{\partial^{3} S}{\partial Q^{2} \partial J}\right)^{2}\left(\frac{\partial^{2} S}{\partial P \partial J}\right)^{2}+\frac{\partial^{2} S}{\partial P^{2}}\left(\frac{\partial^{3} S}{\partial Q \partial J^{2}}\right)^{2}\left(\frac{\partial^{2} S}{\partial Q \partial P}\right)^{2} \\
& +\frac{\partial^{3} S}{\partial P^{3}} \frac{\partial^{2} S}{\partial Q \partial P} \frac{\partial^{2} S}{\partial Q \partial J} \frac{\partial^{3} S}{\partial J^{3}} \frac{\partial^{2} S}{\partial Q^{2}}+\left(\frac{\partial^{3} S}{\partial Q^{2} \partial P}\right)^{2} \frac{\partial^{2} S}{\partial J^{2}}\left(\frac{\partial^{2} S}{\partial P \partial J}\right)^{2} \\
& +\left(\frac{\partial^{3} S}{\partial P^{2} \partial J}\right)^{2} \frac{\partial^{2} S}{\partial Q^{2}}\left(\frac{\partial^{2} S}{\partial Q \partial J}\right)^{2}+\left(\frac{\partial^{3} S}{\partial P \partial J^{2}}\right)^{2} \frac{\partial^{2} S}{\partial Q^{2}}\left(\frac{\partial^{2} S}{\partial Q \partial P}\right)^{2} \\
& +\left(\frac{\partial^{3} S}{\partial Q \partial P^{2}}\right)^{2} \frac{\partial^{2} S}{\partial J^{2}}\left(\frac{\partial^{2} S}{\partial Q \partial J}\right)^{2}+\frac{\partial^{3} S}{\partial P^{3}} \frac{\partial^{2} S}{\partial P \partial J} \frac{\partial^{2} S}{\partial Q \partial J} \frac{\partial^{3} S}{\partial Q^{3}} \frac{\partial^{2} S}{\partial J^{2}} \\
& +\frac{\partial^{3} S}{\partial P^{2} \partial J}\left(\frac{\partial^{2} S}{\partial Q^{2}}\right)^{2} \frac{\partial^{3} S}{\partial P \partial J^{2}} \frac{\partial^{2} S}{\partial P \partial J}+\frac{\partial^{3} S}{\partial Q \partial P^{2}}\left(\frac{\partial^{2} S}{\partial J^{2}}\right)^{2} \frac{\partial^{3} S}{\partial Q^{2} \partial P} \frac{\partial^{2} S}{\partial Q \partial P} \\
& +\frac{\partial^{3} S}{\partial P^{3}} \frac{\partial^{3} S}{\partial P \partial J^{2}}\left(\frac{\partial^{2} S}{\partial Q^{2}}\right)^{2} \frac{\partial^{2} S}{\partial J^{2}}+\frac{\partial^{3} S}{\partial P^{3}} \frac{\partial^{3} S}{\partial Q^{2} \partial P}\left(\frac{\partial^{2} S}{\partial J^{2}}\right)^{2} \frac{\partial^{2} S}{\partial Q^{2}} \\
& +\left(\frac{\partial^{2} S}{\partial P^{2}}\right)^{2} \frac{\partial^{3} S}{\partial Q^{2} \partial J} \frac{\partial^{3} S}{\partial Q \partial J^{2}} \frac{\partial^{2} S}{\partial Q \partial J}+\left(\frac{\partial^{2} S}{\partial P^{2}}\right)^{2} \frac{\partial^{3} S}{\partial Q^{3}} \frac{\partial^{3} S}{\partial Q \partial J^{2}} \frac{\partial^{2} S}{\partial J^{2}} \\
& +\frac{\partial^{2} S}{\partial P^{2}} \frac{\partial^{3} S}{\partial P^{2} \partial J}\left(\frac{\partial^{2} S}{\partial Q^{2}}\right)^{2} \frac{\partial^{3} S}{\partial J^{3}}+\frac{\partial^{2} S}{\partial P^{2}} \frac{\partial^{3} S}{\partial Q \partial P^{2}}\left(\frac{\partial^{2} S}{\partial J^{2}}\right)^{2} \frac{\partial^{3} S}{\partial Q^{3}} \\
&
\end{aligned}
$$




$$
\begin{aligned}
& +\left(\frac{\partial^{2} S}{\partial P^{2}}\right)^{2} \frac{\partial^{2} S}{\partial Q^{2}} \frac{\partial^{3} S}{\partial Q^{2} \partial J} \frac{\partial^{3} S}{\partial J^{3}}, \\
& f_{2}=\left(\frac{\partial^{3} S}{\partial P^{2} \partial J}\right)^{2}\left(\frac{\partial^{2} S}{\partial Q^{2}}\right)^{2} \frac{\partial^{2} S}{\partial J^{2}}+\left(\frac{\partial^{3} S}{\partial Q \partial P^{2}}\right)^{2}\left(\frac{\partial^{2} S}{\partial J^{2}}\right)^{2} \frac{\partial^{2} S}{\partial Q^{2}} \\
& +\frac{\partial^{2} S}{\partial P^{2}} \frac{\partial^{2} S}{\partial Q^{2}} \frac{\partial^{3} S}{\partial Q^{2} \partial P} \frac{\partial^{2} S}{\partial P \partial J} \frac{\partial^{3} S}{\partial J^{3}}+\frac{\partial^{2} S}{\partial P^{2}} \frac{\partial^{3} S}{\partial Q^{2} \partial J} \frac{\partial^{3} S}{\partial Q \partial J^{2}} \frac{\partial^{2} S}{\partial Q \partial P} \frac{\partial^{2} S}{\partial P \partial J} \\
& +\frac{\partial^{2} S}{\partial P^{2}} \frac{\partial^{3} S}{\partial Q^{2} \partial P} \frac{\partial^{2} S}{\partial P \partial J} \frac{\partial^{2} S}{\partial Q \partial J} \frac{\partial^{3} S}{\partial Q \partial J^{2}}+\frac{\partial^{2} S}{\partial P^{2}} \frac{\partial^{3} S}{\partial Q^{2} \partial J} \frac{\partial^{2} S}{\partial Q \partial P} \frac{\partial^{2} S}{\partial Q \partial J} \frac{\partial^{3} S}{\partial P \partial J^{2}} \\
& +\frac{\partial^{2} S}{\partial P^{2}} \frac{\partial^{3} S}{\partial Q^{3}} \frac{\partial^{2} S}{\partial Q \partial P} \frac{\partial^{3} S}{\partial P \partial J^{2}} \frac{\partial^{2} S}{\partial J^{2}}+\frac{\partial^{2} S}{\partial P^{2}} \frac{\partial^{3} S}{\partial Q \partial P^{2}} \frac{\partial^{2} S}{\partial Q \partial J} \frac{\partial^{3} S}{\partial J^{3}} \frac{\partial^{2} S}{\partial Q^{2}} \\
& +\frac{\partial^{2} S}{\partial P^{2}} \frac{\partial^{3} S}{\partial P^{2} \partial J} \frac{\partial^{2} S}{\partial Q \partial J} \frac{\partial^{3} S}{\partial Q^{3}} \frac{\partial^{2} S}{\partial J^{2}}+\frac{\partial^{3} S}{\partial Q \partial P^{2}} \frac{\partial^{2} S}{\partial J^{2}} \frac{\partial^{3} S}{\partial Q^{2} \partial P} \frac{\partial^{2} S}{\partial P \partial J} \frac{\partial^{2} S}{\partial Q \partial J} \\
& +\frac{\partial^{3} S}{\partial P^{2} \partial J} \frac{\partial^{2} S}{\partial Q^{2}} \frac{\partial^{3} S}{\partial P \partial J^{2}} \frac{\partial^{2} S}{\partial Q \partial P} \frac{\partial^{2} S}{\partial Q \partial J}+\frac{\partial^{3} S}{\partial Q \partial P^{2}} \frac{\partial^{2} S}{\partial P \partial J} \frac{\partial^{2} S}{\partial Q \partial J} \frac{\partial^{3} S}{\partial P \partial J^{2}} \frac{\partial^{2} S}{\partial Q^{2}} \\
& +\frac{\partial^{3} S}{\partial P^{3}} \frac{\partial^{2} S}{\partial P \partial J} \frac{\partial^{3} S}{\partial Q^{2} \partial J} \frac{\partial^{2} S}{\partial Q^{2}} \frac{\partial^{2} S}{\partial J^{2}}+\frac{\partial^{3} S}{\partial P^{3}} \frac{\partial^{2} S}{\partial Q \partial P} \frac{\partial^{3} S}{\partial Q \partial J^{2}} \frac{\partial^{2} S}{\partial Q^{2}} \frac{\partial^{2} S}{\partial J^{2}} \\
& +\frac{\partial^{3} S}{\partial P^{2} \partial J} \frac{\partial^{2} S}{\partial Q \partial J} \frac{\partial^{3} S}{\partial Q^{2} \partial P} \frac{\partial^{2} S}{\partial Q \partial P} \frac{\partial^{2} S}{\partial J^{2}}+\frac{\partial^{3} S}{\partial P^{2} \partial J} \frac{\partial^{2} S}{\partial Q \partial P} \frac{\partial^{2} S}{\partial P \partial J} \frac{\partial^{3} S}{\partial Q \partial J^{2}} \frac{\partial^{2} S}{\partial Q^{2}} \\
& +\frac{\partial^{3} S}{\partial Q \partial P^{2}} \frac{\partial^{2} S}{\partial J^{2}} \frac{\partial^{3} S}{\partial Q^{2} \partial J} \frac{\partial^{2} S}{\partial Q \partial P} \frac{\partial^{2} S}{\partial P \partial J}+\frac{\partial^{2} S}{\partial P^{2}}\left(\frac{\partial^{3} S}{\partial Q \partial P \partial J}\right)^{2}\left(\frac{\partial^{2} S}{\partial Q \partial J}\right)^{2} \\
& +\frac{\partial^{2} S}{\partial P^{2}}\left(\frac{\partial^{3} S}{\partial Q^{2} \partial P}\right)^{2}\left(\frac{\partial^{2} S}{\partial J^{2}}\right)^{2}+\left(\frac{\partial^{2} S}{\partial P^{2}}\right)^{2}\left(\frac{\partial^{3} S}{\partial Q^{2} \partial J}\right)^{2} \frac{\partial^{2} S}{\partial J^{2}} \\
& +\left(\frac{\partial^{2} S}{\partial P^{2}}\right)^{2} \frac{\partial^{2} S}{\partial Q^{2}}\left(\frac{\partial^{3} S}{\partial Q \partial J^{2}}\right)^{2}+\left(\frac{\partial^{3} S}{\partial Q \partial P \partial J}\right)^{2}\left(\frac{\partial^{2} S}{\partial Q \partial P}\right)^{2} \frac{\partial^{2} S}{\partial J^{2}} \\
& +\left(\frac{\partial^{3} S}{\partial Q \partial P \partial J}\right)^{2}\left(\frac{\partial^{2} S}{\partial P \partial J}\right)^{2} \frac{\partial^{2} S}{\partial Q^{2}}+\frac{\partial^{2} S}{\partial P^{2}}\left(\frac{\partial^{3} S}{\partial P \partial J^{2}}\right)^{2}\left(\frac{\partial^{2} S}{\partial Q^{2}}\right)^{2} \\
& +\frac{\partial^{3} S}{\partial P^{3}} \frac{\partial^{3} S}{\partial P \partial J^{2}} \frac{\partial^{2} S}{\partial Q^{2}}\left(\frac{\partial^{2} S}{\partial Q \partial J}\right)^{2}+\frac{\partial^{3} S}{\partial Q \partial P^{2}} \frac{\partial^{2} S}{\partial J^{2}} \frac{\partial^{3} S}{\partial Q^{3}}\left(\frac{\partial^{2} S}{\partial P \partial J}\right)^{2} \\
& +\frac{\partial^{3} S}{\partial P^{3}} \frac{\partial^{2} S}{\partial P \partial J}\left(\frac{\partial^{2} S}{\partial Q^{2}}\right)^{2} \frac{\partial^{3} S}{\partial J^{3}}+\frac{\partial^{3} S}{\partial P^{3}} \frac{\partial^{3} S}{\partial Q^{2} \partial P} \frac{\partial^{2} S}{\partial J^{2}}\left(\frac{\partial^{2} S}{\partial Q \partial J}\right)^{2} \\
& +\frac{\partial^{3} S}{\partial P^{3}} \frac{\partial^{2} S}{\partial Q \partial P}\left(\frac{\partial^{2} S}{\partial J^{2}}\right)^{2} \frac{\partial^{3} S}{\partial Q^{3}}+\frac{\partial^{3} S}{\partial P^{2} \partial J} \frac{\partial^{2} S}{\partial Q^{2}} \frac{\partial^{3} S}{\partial J^{3}}\left(\frac{\partial^{2} S}{\partial Q \partial P}\right)^{2} \\
& +\left(\frac{\partial^{2} S}{\partial P^{2}}\right)^{2} \frac{\partial^{3} S}{\partial Q^{3}} \frac{\partial^{2} S}{\partial Q \partial J} \frac{\partial^{3} S}{\partial J^{3}}+\frac{\partial^{2} S}{\partial P^{2}} \frac{\partial^{3} S}{\partial Q^{2} \partial J} \frac{\partial^{3} S}{\partial J^{3}}\left(\frac{\partial^{2} S}{\partial Q \partial P}\right)^{2} \\
& +\frac{\partial^{2} S}{\partial P^{2}} \frac{\partial^{3} S}{\partial Q^{3}} \frac{\partial^{3} S}{\partial Q \partial J^{2}}\left(\frac{\partial^{2} S}{\partial P \partial J}\right)^{2}, \\
& f_{3}=\frac{\partial^{2} S}{\partial P^{2}} \frac{\partial^{2} S}{\partial Q^{2}} \frac{\partial^{3} S}{\partial Q \partial J^{2}} \frac{\partial^{2} S}{\partial Q \partial P} \frac{\partial^{3} S}{\partial P \partial J^{2}}+\frac{\partial^{2} S}{\partial P^{2}} \frac{\partial^{3} S}{\partial Q^{2} \partial P} \frac{\partial^{2} S}{\partial P \partial J} \frac{\partial^{3} S}{\partial Q^{2} \partial J} \frac{\partial^{2} S}{\partial J^{2}} \\
& +\frac{\partial^{3} S}{\partial P^{2} \partial J} \frac{\partial^{3} S}{\partial Q \partial P^{2}} \frac{\partial^{2} S}{\partial Q \partial J} \frac{\partial^{2} S}{\partial Q^{2}} \frac{\partial^{2} S}{\partial J^{2}}+\frac{\partial^{3} S}{\partial Q \partial P \partial J}\left(\frac{\partial^{2} S}{\partial Q \partial P}\right)^{3} \frac{\partial^{3} S}{\partial J^{3}} \\
& +\frac{\partial^{3} S}{\partial Q \partial P \partial J}\left(\frac{\partial^{2} S}{\partial P \partial J}\right)^{3} \frac{\partial^{3} S}{\partial Q^{3}}+\frac{\partial^{3} S}{\partial P^{3}} \frac{\partial^{3} S}{\partial Q \partial P \partial J}\left(\frac{\partial^{2} S}{\partial Q \partial J}\right)^{3} \\
& +\frac{\partial^{3} S}{\partial Q \partial P^{2}} \frac{\partial^{3} S}{\partial Q \partial J^{2}}\left(\frac{\partial^{2} S}{\partial Q \partial P}\right)^{2} \frac{\partial^{2} S}{\partial J^{2}}+\frac{\partial^{3} S}{\partial P^{2} \partial J} \frac{\partial^{3} S}{\partial Q^{2} \partial J}\left(\frac{\partial^{2} S}{\partial P \partial J}\right)^{2} \frac{\partial^{2} S}{\partial Q^{2}} \\
& +\frac{\partial^{2} S}{\partial Q^{2}} \frac{\partial^{3} S}{\partial Q^{2} \partial P}\left(\frac{\partial^{2} S}{\partial P \partial J}\right)^{2} \frac{\partial^{3} S}{\partial P \partial J^{2}}+\frac{\partial^{2} S}{\partial P^{2}} \frac{\partial^{3} S}{\partial P^{2} \partial J} \frac{\partial^{3} S}{\partial Q^{2} \partial J}\left(\frac{\partial^{2} S}{\partial Q \partial J}\right)^{2} \\
& +\frac{\partial^{2} S}{\partial P^{2}} \frac{\partial^{3} S}{\partial Q \partial P^{2}} \frac{\partial^{3} S}{\partial Q \partial J^{2}}\left(\frac{\partial^{2} S}{\partial Q \partial J}\right)^{2}+\frac{\partial^{3} S}{\partial Q^{2} \partial P} \frac{\partial^{3} S}{\partial P \partial J^{2}}\left(\frac{\partial^{2} S}{\partial Q \partial P}\right)^{2} \frac{\partial^{2} S}{\partial J^{2}}, \\
& f_{4}=\frac{\partial^{2} S}{\partial P^{2}} \frac{\partial^{2} S}{\partial Q^{2}} \frac{\partial^{3} S}{\partial Q \partial P \partial J} \frac{\partial^{2} S}{\partial Q \partial P} \frac{\partial^{3} S}{\partial J^{3}}+\frac{\partial^{2} S}{\partial P^{2}} \frac{\partial^{3} S}{\partial Q \partial P \partial J} \frac{\partial^{2} S}{\partial Q \partial P} \frac{\partial^{2} S}{\partial Q \partial J} \frac{\partial^{3} S}{\partial Q \partial J^{2}} \\
& +\frac{\partial^{2} S}{\partial P^{2}} \frac{\partial^{3} S}{\partial Q^{2} \partial J} \frac{\partial^{2} S}{\partial P \partial J} \frac{\partial^{3} S}{\partial Q \partial P \partial J} \frac{\partial^{2} S}{\partial Q \partial J}+\frac{\partial^{3} S}{\partial Q^{2} \partial P} \frac{\partial^{2} S}{\partial J^{2}} \frac{\partial^{3} S}{\partial Q \partial P \partial J} \frac{\partial^{2} S}{\partial Q \partial P} \frac{\partial^{2} S}{\partial P \partial J} \\
& +\frac{\partial^{3} S}{\partial Q \partial P \partial J} \frac{\partial^{2} S}{\partial Q \partial P} \frac{\partial^{2} S}{\partial P \partial J} \frac{\partial^{3} S}{\partial P \partial J^{2}} \frac{\partial^{2} S}{\partial Q^{2}}+\frac{\partial^{3} S}{\partial Q^{2} \partial P} \frac{\partial^{3} S}{\partial P \partial J^{2}} \frac{\partial^{2} S}{\partial Q \partial P} \frac{\partial^{2} S}{\partial P \partial J} \frac{\partial^{2} S}{\partial Q \partial J}
\end{aligned}
$$




$$
\begin{aligned}
& +\frac{\partial^{3} S}{\partial P^{3}} \frac{\partial^{3} S}{\partial Q \partial P \partial J} \frac{\partial^{2} S}{\partial Q \partial J} \frac{\partial^{2} S}{\partial Q^{2}} \frac{\partial^{2} S}{\partial J^{2}}+\frac{\partial^{3} S}{\partial P^{2} \partial J} \frac{\partial^{2} S}{\partial Q \partial J} \frac{\partial^{3} S}{\partial Q \partial P \partial J} \frac{\partial^{2} S}{\partial P \partial J} \frac{\partial^{2} S}{\partial Q^{2}} \\
& +\frac{\partial^{3} S}{\partial Q \partial P^{2}} \frac{\partial^{2} S}{\partial J^{2}} \frac{\partial^{3} S}{\partial Q \partial P \partial J} \frac{\partial^{2} S}{\partial Q \partial P} \frac{\partial^{2} S}{\partial Q \partial J}+\frac{\partial^{3} S}{\partial P^{2} \partial J} \frac{\partial^{3} S}{\partial Q^{2} \partial J} \frac{\partial^{2} S}{\partial Q \partial P} \frac{\partial^{2} S}{\partial P \partial J} \frac{\partial^{2} S}{\partial Q \partial J} \\
& +\frac{\partial^{3} S}{\partial Q \partial P^{2}} \frac{\partial^{3} S}{\partial Q \partial J^{2}} \frac{\partial^{2} S}{\partial Q \partial P} \frac{\partial^{2} S}{\partial P \partial J} \frac{\partial^{2} S}{\partial Q \partial J}+\frac{\partial^{3} S}{\partial Q \partial J^{2}}\left(\frac{\partial^{2} S}{\partial Q \partial P}\right)^{3} \frac{\partial^{3} S}{\partial P \partial J^{2}} \\
& +\frac{\partial^{3} S}{\partial Q^{2} \partial P}\left(\frac{\partial^{2} S}{\partial P \partial J}\right)^{3} \frac{\partial^{3} S}{\partial Q^{2} \partial J}+\frac{\partial^{3} S}{\partial P^{2} \partial J} \frac{\partial^{3} S}{\partial Q \partial P^{2}}\left(\frac{\partial^{2} S}{\partial Q \partial J}\right)^{3} \\
& +\frac{\partial^{3} S}{\partial Q \partial P \partial J} \frac{\partial^{2} S}{\partial Q \partial J} \frac{\partial^{3} S}{\partial P \partial J^{2}}\left(\frac{\partial^{2} S}{\partial Q \partial P}\right)^{2}+\frac{\partial^{3} S}{\partial Q^{2} \partial P}\left(\frac{\partial^{2} S}{\partial P \partial J}\right)^{2} \frac{\partial^{3} S}{\partial Q \partial P \partial J} \frac{\partial^{2} S}{\partial Q \partial J} \\
& +\frac{\partial^{3} S}{\partial P^{3}} \frac{\partial^{2} S}{\partial P \partial J} \frac{\partial^{3} S}{\partial Q^{2} \partial J}\left(\frac{\partial^{2} S}{\partial Q \partial J}\right)^{2}+\frac{\partial^{3} S}{\partial P^{3}} \frac{\partial^{2} S}{\partial Q \partial P} \frac{\partial^{3} S}{\partial Q \partial J^{2}}\left(\frac{\partial^{2} S}{\partial Q \partial J}\right)^{2} \\
& +\frac{\partial^{3} S}{\partial P^{2} \partial J} \frac{\partial^{2} S}{\partial Q \partial J} \frac{\partial^{3} S}{\partial Q^{3}}\left(\frac{\partial^{2} S}{\partial P \partial J}\right)^{2}+\frac{\partial^{3} S}{\partial P^{2} \partial J}\left(\frac{\partial^{2} S}{\partial Q \partial J}\right)^{2} \frac{\partial^{3} S}{\partial Q \partial P \partial J} \frac{\partial^{2} S}{\partial Q \partial P} \\
& +\frac{\partial^{3} S}{\partial Q \partial P \partial J} \frac{\partial^{3} S}{\partial Q^{2} \partial J}\left(\frac{\partial^{2} S}{\partial P \partial J}\right)^{2} \frac{\partial^{2} S}{\partial Q \partial P}+\frac{\partial^{3} S}{\partial Q^{2} \partial P} \frac{\partial^{2} S}{\partial P \partial J} \frac{\partial^{3} S}{\partial J^{3}}\left(\frac{\partial^{2} S}{\partial Q \partial P}\right)^{2} \\
& +\frac{\partial^{3} S}{\partial Q \partial P \partial J} \frac{\partial^{2} S}{\partial P \partial J} \frac{\partial^{3} S}{\partial Q \partial J^{2}}\left(\frac{\partial^{2} S}{\partial Q \partial P}\right)^{2}+\frac{\partial^{3} S}{\partial Q \partial P^{2}}\left(\frac{\partial^{2} S}{\partial Q \partial J}\right)^{2} \frac{\partial^{3} S}{\partial Q \partial P \partial J} \frac{\partial^{2} S}{\partial P \partial J} \\
& +\frac{\partial^{3} S}{\partial Q^{3}} \frac{\partial^{2} S}{\partial Q \partial P} \frac{\partial^{3} S}{\partial P \partial J^{2}}\left(\frac{\partial^{2} S}{\partial P \partial J}\right)^{2}+\frac{\partial^{3} S}{\partial Q \partial P^{2}} \frac{\partial^{2} S}{\partial Q \partial J} \frac{\partial^{3} S}{\partial J^{3}}\left(\frac{\partial^{2} S}{\partial Q \partial P}\right)^{2} \\
& +\frac{\partial^{2} S}{\partial P^{2}} \frac{\partial^{3} S}{\partial Q \partial P \partial J} \frac{\partial^{2} S}{\partial P \partial J} \frac{\partial^{3} S}{\partial Q^{3}} \frac{\partial^{2} S}{\partial J^{2}}, \\
& f_{5}=\frac{\partial^{2} S}{\partial P^{2}} \frac{\partial^{3} S}{\partial Q^{3}} \frac{\partial^{2} S}{\partial P \partial J} \frac{\partial^{2} S}{\partial Q \partial J} \frac{\partial^{3} S}{\partial P \partial J^{2}}+\frac{\partial^{2} S}{\partial P^{2}} \frac{\partial^{3} S}{\partial Q^{2} \partial P} \frac{\partial^{2} S}{\partial Q \partial P} \frac{\partial^{2} S}{\partial Q \partial J} \frac{\partial^{3} S}{\partial J^{3}} \\
& +\frac{\partial^{3} S}{\partial P^{3}} \frac{\partial^{2} S}{\partial P \partial J} \frac{\partial^{2} S}{\partial Q \partial J} \frac{\partial^{3} S}{\partial Q \partial J^{2}} \frac{\partial^{2} S}{\partial Q^{2}}+\frac{\partial^{3} S}{\partial P^{3}} \frac{\partial^{2} S}{\partial Q \partial P} \frac{\partial^{2} S}{\partial J^{2}} \frac{\partial^{3} S}{\partial Q^{2} \partial J} \frac{\partial^{2} S}{\partial Q \partial J} \\
& +\frac{\partial^{3} S}{\partial P^{2} \partial J} \frac{\partial^{2} S}{\partial Q \partial P} \frac{\partial^{2} S}{\partial P \partial J} \frac{\partial^{3} S}{\partial Q^{3}} \frac{\partial^{2} S}{\partial J^{2}}+\frac{\partial^{3} S}{\partial Q \partial P^{2}} \frac{\partial^{2} 3 S}{\partial Q \partial P} \frac{\partial^{2} S}{\partial P \partial J} \frac{\partial^{3} S}{\partial J^{3}} \frac{\partial^{2} S}{\partial Q^{2}}, \\
& f_{6}=\frac{\partial^{2} S}{\partial P^{2}} \frac{\partial^{2} S}{\partial Q^{2}} \frac{\partial^{3} S}{\partial P \partial J^{2}} \frac{\partial^{3} S}{\partial Q^{2} \partial J} \frac{\partial^{2} S}{\partial P \partial J}+\frac{\partial^{2} S}{\partial P^{2}} \frac{\partial^{3} S}{\partial Q^{2} \partial P} \frac{\partial^{2} S}{\partial Q \partial P} \frac{\partial^{3} S}{\partial Q \partial J^{2}} \frac{\partial^{2} S}{\partial J^{2}} \\
& +\frac{\partial^{2} S}{\partial P^{2}} \frac{\partial^{3} S}{\partial P^{2} \partial J} \frac{\partial^{2} S}{\partial Q \partial J} \frac{\partial^{3} S}{\partial Q \partial J^{2}} \frac{\partial^{2} S}{\partial Q^{2}}+\frac{\partial^{2} S}{\partial P^{2}} \frac{\partial^{3} S}{\partial Q \partial P^{2}} \frac{\partial^{2} S}{\partial J^{2}} \frac{\partial^{3} S}{\partial Q^{2} \partial J} \frac{\partial^{2} S}{\partial Q \partial J} \\
& +\frac{\partial^{3} S}{\partial P^{2} \partial J} \frac{\partial^{3} S}{\partial Q^{2} \partial P} \frac{\partial^{2} S}{\partial P \partial J} \frac{\partial^{2} S}{\partial Q^{2}} \frac{\partial^{2} S}{\partial J^{2}}+\frac{\partial^{3} S}{\partial Q \partial P^{2}} \frac{\partial^{2} S}{\partial Q \partial P} \frac{\partial^{3} S}{\partial P \partial J^{2}} \frac{\partial^{2} S}{\partial Q^{2}} \frac{\partial^{2} S}{\partial J^{2}} \\
& +\frac{\partial^{3} S}{\partial Q \partial P^{2}} \frac{\partial^{3} S}{\partial Q \partial J^{2}}\left(\frac{\partial^{2} S}{\partial P \partial J}\right)^{2} \frac{\partial^{2} S}{\partial Q^{2}}+\frac{\partial^{3} S}{\partial P^{2} \partial J} \frac{\partial^{3} S}{\partial Q^{2} \partial J}\left(\frac{\partial^{2} S}{\partial Q \partial P}\right)^{2} \frac{\partial^{2} S}{\partial J^{2}} \\
& +\frac{\partial^{2} S}{\partial P^{2}} \frac{\partial^{3} S}{\partial Q^{2} \partial P} \frac{\partial^{3} S}{\partial P \partial J^{2}}\left(\frac{\partial^{2} S}{\partial Q \partial J}\right)^{2}+\frac{\partial^{2} S}{\partial P^{2}}\left(\frac{\partial^{3} S}{\partial Q \partial P \partial J}\right)^{2} \frac{\partial^{2} S}{\partial Q^{2}} \frac{\partial^{2} S}{\partial J^{2}}, \\
& f_{7}=\frac{\partial^{2} S}{\partial P^{2}} \frac{\partial^{2} S}{\partial Q^{2}} \frac{\partial^{3} S}{\partial Q \partial P \partial J} \frac{\partial^{3} S}{\partial Q \partial J^{2}} \frac{\partial^{2} S}{\partial P \partial J}+\frac{\partial^{2} S}{\partial P^{2}} \frac{\partial^{3} S}{\partial Q^{2} \partial J} \frac{\partial^{2} S}{\partial Q \partial P} \frac{\partial^{3} S}{\partial Q \partial P \partial J} \frac{\partial^{2} S}{\partial J^{2}} \\
& +\frac{\partial^{2} S(}{\partial P^{2}} \frac{\partial^{3} S}{\partial Q \partial P \partial J} \frac{\partial^{2} S}{\partial Q \partial J} \frac{\partial^{3} S}{\partial P \partial J^{2}} \frac{\partial^{2} S}{\partial Q^{2}}+\frac{\partial^{2} S}{\partial P^{2}} \frac{\partial^{3} S}{\partial Q^{2} \partial P} \frac{\partial^{2} S}{\partial J^{2}} \frac{\partial^{3} S}{\partial Q \partial P \partial J} \frac{\partial^{2} S}{\partial Q \partial J} \\
& +\frac{\partial^{3} S}{\partial P^{2} \partial J} \frac{\partial^{2} S}{\partial Q \partial P} \frac{\partial^{3} S}{\partial Q \partial P \partial J} \frac{\partial^{2} S}{\partial Q^{2}} \frac{\partial^{2} S}{\partial J^{2}}+\frac{\partial^{3} S}{\partial Q \partial P^{2}} \frac{\partial^{2} S}{\partial P \partial J} \frac{\partial^{3} S}{\partial Q \partial P \partial J} \frac{\partial^{2} S}{\partial Q^{2}} \frac{\partial^{2} S}{\partial J^{2}} \\
& +\frac{\partial^{3} S}{\partial Q \partial P^{2}} \frac{\partial^{2} S}{\partial Q \partial P} \frac{\partial^{3} S}{\partial P \partial J^{2}}\left(\frac{\partial^{2} S}{\partial Q \partial J}\right)^{2}+\frac{\partial^{3} S}{\partial P^{2} \partial J} \frac{\partial^{3} S}{\partial Q^{2} \partial P} \frac{\partial^{2} S}{\partial P \partial J}\left(\frac{\partial^{2} S}{\partial Q \partial J}\right)^{2} \\
& +\frac{\partial^{3} S}{\partial P^{2} \partial J} \frac{\partial^{2} S}{\partial Q \partial J} \frac{\partial^{3} S}{\partial Q \partial J^{2}}\left(\frac{\partial^{2} S}{\partial Q \partial P}\right)^{2}+\frac{\partial^{3} S}{\partial Q \partial P^{2}}\left(\frac{\partial^{2} S}{\partial P \partial J}\right)^{2} \frac{\partial^{3} S}{\partial Q^{2} \partial J} \frac{\partial^{2} S}{\partial Q \partial J} \\
& +\frac{\partial^{3} S}{\partial Q^{2} \partial P} \frac{\partial^{2} S}{\partial Q \partial P} \frac{\partial^{3} S}{\partial Q \partial J^{2}}\left(\frac{\partial^{2} S}{\partial P \partial J}\right)^{2}+\frac{\partial^{3} S}{\partial Q^{2} \partial J} \frac{\partial^{2} S}{\partial P \partial J} \frac{\partial^{3} S}{\partial P \partial J^{2}}\left(\frac{\partial^{2} S}{\partial Q \partial P}\right)^{2}, \\
& f_{8}=\left(\frac{\partial^{3} S}{\partial Q \partial P \partial J}\right)^{2} \frac{\partial^{2} S}{\partial Q \partial P} \frac{\partial^{2} S}{\partial P \partial J} \frac{\partial^{2} S}{\partial Q \partial J}
\end{aligned}
$$

\section{Acknowledgement}


This work has been supported in part by the European Research Council grant n. 226455, "SUPERSYMMETRY, QUANTUM GRAVITY AND GAUGE FIELDS (SUPERFIELDS)". B. N. T. would like to thank Prof. A. Sen and Prof. R. Emparan and Prof. S. Minwalla for necessary discussions on stability of the rotating black hole configurations and possible phase transions during the "Spring School on Superstring Theory and Related Topics-2008, ICTP Trieste, Italy"; Prof. R. Gopakumar for valuable explanation on black brane configurations and their degeneracy of microstates during the "National Conference on New Trends in Field Theories, Nov. 2008, Banaras Hindu University, Varanasi, India"; Prof. V. Ravishankar, Prof. P. Jain, Prof. U. B. Tewari, Prof M. K. Harbola, and Prof. R. K. Thareja for providing their viable support during the preparation of this manuscript; Vinod Chandra for ample discussions on intrinsic geometric character of Hot QCD and related configurations. B. N. T. further acknowledges CSIR, New Delhi (India) for the financial support under the research grant "CSIR-SRF-9/92(343)/2004-EMR-I".

\section{References}

[1] A. Strominger, C. Vafa, "Microscopic Origin of the Bekenstein-Hawking Entropy", Phys. Lett. B 379, 99-104, (1996), arXiv:hep-th/9601029v2.

[2] C. G. Callan, J. M. Maldacena, "D-brane Approach to Black Hole Quantum Mechanics", Nucl.Phys. B 472 (1996) 591-610, arXiv:hep-th/9602043v2.

[3] S. D. Mathur, "Black hole size and phase space volumes", arXiv:0706.3884v1 [hep-th] .

[4] J. M. Maldacena, "The Large N Limit of Superconformal Field Theories and Supergravity", Adv. Theor. Math. Phys. 2 (1998) 231-252; arXiv:hep-th/9711200 r3.

[5] J. M. Maldacena, "Gravity, particle physics and their unification", Int. J. Theor. Phys. 38 (1999) 1113-1133, arXiv:hep-ph/0002092v1.

[6] S. Bellucci, E. Ivanov, S. Krivonos, "AdS/CFT Equivalence Transformation", Phys. Rev. D 66 (2002) 086001; Erratum-ibid. D67 (2003) 049901, arXiv:hep-th/0206126.

[7] S. Bellucci, A. Galajinsky, E. Ivanov, S. Krivonos, " $A d S_{2} / C F T_{1}$, Canonical Transformations and Superconformal Mechanics", Phys.Lett. B 555 (2003) 99-106, arXiv:hep-th/0212204.

[8] S. W. Hawking, "Black Holes in General Relativity", Commun. Math. Phys. 25, 152-166, 1972.

[9] D. C Robinson, "Uniqueness of the Kerr black hole", Phys. Rev. Lett. 34, 905-906, 1975.

[10] P. O. Mazur, "Proof of Uniqueness of the Kerr-Newman Black Hole solution", J. Phys. A 15, 3173-3180, 1982.

[11] S. D. Mathur, "Where are the states of a black hole", OHSTPY-HEP-T-04-001, arXiv:hep-th/0401115

[12] S. D. Mathur, A. Saxena, Y. K. Srivastava, "Constructing 'hair' for the three charge hole", arXiv:hep-th/0311092

[13] R. Emparan, H. S. Reall, "A rotating black ring in five dimensions", Phys. Rev. Lett. 88 (2002) 101101, arXiv:hep-th/0110260

[14] R. C. Myers, M.J. Perry, "Black Holes In Higher Dimensional Space-Times", Ann. Phys. 172 (1986) 304.

[15] G. W. Gibbons, D. L. Wiltshire, "Black Holes In Kaluza-Klein Theory", Ann. Phys. 167 (1986) 201, [Erratum-ibid. 1761987 393].

[16] N. Itzhaki, "D6 + D0 and five-dimensional spinning black hole", JHEP 9809 (1998) 018, arXiv:hep-th/9809063

[17] V. Balasubramanian, F. Larsen, "On D-Branes and Black Holes in Four Dimensions", Nucl. Phys. B 478, 199 (1996), arXiv:hep-th/9604189

[18] B. N. Tiwari, "Sur les corrections de la géométrie thermodynamique des trous noirs", Proc. Quantum Gravity, Hoelback 2008, arXiv:0801.4087v1 [hep-th].

[19] H. Lin, O. Lunin, J.M. Maldacena, "Bubbling AdS space and 1/2 BPS geometries", JHEP 0410, 025 (2004), arXiv:hep-th/0409174

[20] D. Berenstein, "A toy model for the AdS/CFT correspondence", JHEP 0407, 018 (2004), arXiv:hep-th/0403110

[21] N. V. Suryanarayana, "Half-BPS giants, free fermions and microstates of superstars", JHEP 0601, 082 (2006), arXiv:hep-th/0411145 
[22] M. M. Sheikh-Jabbari, M. Torabian, "Classification of all 1/2 BPS solutions of the tiny graviton matrix theory", JHEP 0504, 001 (2005), arXiv:hep-th/0501001.

[23] G. Mandal, "Fermions from half-BPS supergravity", JHEP 0508, 052 (2005), arXiv:hep-th/0502104

[24] L. Grant, L. Maoz, J. Marsano, K. Papadodimas, V.S. Rychkov, "Minisuperspace quantization of 'Bubbling AdS' and free fermion droplets", JHEP 0508, 025 (2005), arXiv:hep-th/0505079

[25] A. Dhar, G. Mandal, M. Smedback, "From gravitons to giants", JHEP 0603, 031 (2006), arXiv:hep-th/0512312

[26] J. B. Gutowski, H. S. Reall, "Supersymmetric AdS 5 black holes", JHEP 0402, 006 (2004), arXiv:hep-th/0401042

[27] J. B. Gutowski, H. S. Reall, "General supersymmetric AdS 5 black holes", JHEP 0404, 048 (2004), arXiv:hep-th/0401129.

[28] Z. W. Chong, M. Cvetic, H. Lu, C. N. Pope, "Five-dimensional gauged supergravity black holes with independent rotation parameters", Phys. Rev. D 72, 041901 (2005), arXiv:hep-th/0505112.

[29] Z. W. Chong, M. Cvetic, H. Lu, C. N. Pope, "General non-extremal rotating black holes in minimal five-dimensional gauged supergravity", Phys. Rev. Lett. 95, 161301 (2005), arXiv:hep-th/0506029

[30] H. K. Kunduri, J. Lucietti, H. S. Reall, "Supersymmetric multi-charge AdS 5 black holes", JHEP 0604, 036 (2006), arXiv:hep-th/0601156.

[31] R. C. Myers, O. Tafjord, "Superstars and giant gravitons", JHEP 0111, 009 (2001), arXiv:hep-th/0109127

[32] K. Behrndt, A. H. Chamseddine, W. A. Sabra, "BPS black holes in N=2 five dimensional AdS supergravity", Phys. Lett. B 442, 97 (1998), arXiv:hep-th/9807187.

[33] J. Kinney, J. M. Maldacena, S. Minwalla, S. Raju, "An index for 4 dimensional super conformal theories", arXiv:hep-th/0510251.

[34] M. Berkooz, D. Reichmann, J. Simon, "A Fermi surface model for large supersymmetric AdS(5) black holes", arXiv:hep-th/0604023

[35] S. Kim, K. M. Lee, "1/16-BPS Black Holes and Giant Gravitons in the $A d S_{5} \times S^{5}$ Space", JHEP 0612, 077, 2006, arXiv:hep-th/0607085 v2.

[36] O. Lunin, S. D. Mathur, "AdS/CFT Duality and the Black Hole Information Paradox", Nucl. Phys. B 623 (2002) 342, arXiv:hep-th/0109154

[37] S. D. Mathur, "The fuzzball proposal for black holes: An elementary review", Fortsch. Phys. 53 (2005) 793, arXiv:hep-th/0502050

[38] S. Bellucci, B. N. Tiwari, On the Microscopic Perspective of Black Branes Thermodynamic Geometry, Entropy 2010, 12, 2097-2143, arXiv:0808.3921v1 [hep-th].

[39] O. Lunin, S. D. Mathur, "Metric of the multiply wound rotating string", Nucl. Phys. B 610, 49 (2001), arXiv:hep-th/0105136

[40] J. C. Breckenridge, D. A. Lowe, R. C. Myers, A. W. Peet, A. Strominger and C. Vafa, "Macroscopic and Microscopic Entropy of Near-Extremal Spinning Black Holes", Phys. Lett. B 381, 423 (1996), arXiv:hep-th/9603078

[41] C. A. R. Herdeiro, "Special properties of five dimensional BPS rotating black holes", Nucl. Phys. B 582, 363 (2000) arXiv:hep-th/0003063

[42] H. Elvang, "A charged rotating black ring", Phys. Rev. D 68 (2003) 124016, arXiv:hep-th/0305247.

[43] H. Elvang, R. Emparan, "Black rings, supertubes, and a stringy resolution of black hole nonuniqueness", JHEP 0311 (2003) 035, arXiv:hep-th/0310008.

[44] S. Ferrara, R. Kallosh, A. Strominger: "N=2 Extremal Black Holes", Phys. Rev. D 52 (1995) 5412-5416, arXiv:hep-th/9508072 v3.

[45] A. Strominger, "Macroscopic Entropy of $N=2$ Extremal Black Holes", Phys. Lett. B 383 (1996) 39-43, arXiv:hep-th/9602111 3 .

[46] J. M. Maldacena, "Black Holes in String Theory", Ph. D. Thesis, Princeton University, June 1996, arXiv:hep-th/9607235

[47] J. M. Maldacena, A. Strominger, E. Witten, "Black Hole Entropy in M-Theory", JHEP 9712, 002 (1997), arXiv:hep-th/9711053

[48] H. Elvang, R. Emparan, D. Mateos, H. S. Reall, "Supersymmetric black rings and three-charge supertubes", Phys. Rev. D 71, 024033 (2005), arXiv:hep-th/0408120 
[49] O. Aharony, S. S. Gubser, J. Maldacena, H. Ooguri, Y. Oz: "Large N Field Theories, String Theory and Gravity", Phys. Rept. 323 (2000) 183-386, arXiv:hep-th/9905111 v3.

[50] G. Moore, "Introduction to modular functions and their application to 2D CFT", Spring School on Superstring Theory and Related Topics-2008, ICTP Trieste, Italy; http://cdsagenda5.ictp.trieste.it.

[51] M. R. Gaberdiel, S. Gukov, C. A. Keller, G. W. Moore, H. Ooguri, "Extremal N=(2,2) 2D Conformal Field Theories and Constraints of Modularity", arXiv:0805.4216v1 [hep-th].

[52] A. Sen, "Entropy Function and $\operatorname{AdS}(2) / C F T(1)$ Correspondence", arXiv:0805.0095 3 [hep-th].

[53] R. K. Gupta, A. Sen, "Ads(3)/CFT(2) to Ads(2)/CFT(1)", arXiv:0806.0053v2 [hep-th].

[54] D. Gaiotto, Xi Yin, "Genus Two Partition Functions of Extremal Conformal Field Theories", arXiv:0707.3437v1 [hep-th].

[55] A. Belhaj, "On Black Objects in Type IIA Superstring Theory on Calabi-Yau Manifolds", African Journal of Mathematical Physics, Volume 6(2008)49-54 arXiv:0809.1114v2 [hep-th]

[56] G. Lopes Cardoso, B. de Wit, J. Kappeli, T. Mohaupt, "Asymptotic degeneracy of dyonic N = 4 string states and black hole entropy", JHEP 0412, 075 (2004), arXiv: hep-th/0412287

[57] A. Sen, "Black Holes and the Spectrum of Half-BPS States in N=4 Supersymmetric String Theory", Adv. Theor. Math. Phys. 9 (2005) 527-558, arXiv:hep-th/0504005v2

[58] F. Weinhold, "Metric geometry of equilibrium thermodynamics", J. Chem. Phys. 63 , 2479 (1975), DOI:10.1063/1.431689.

[59] F. Weinhold, "Metric geometry of equilibrium thermodynamics. II", Scaling, homogeneity, and generalized GibbsDuhem relations, ibid J. Chem. Phys 63, 2484 ( 1975).

[60] G. Ruppeiner, "Riemannian geometry in thermodynamic fluctuation theory", Rev. Mod. Phys 67 (1995) 605, Erratum 68 (1996) 313.

[61] G. Ruppeiner, "Thermodynamics: A Riemannian geometric model", Phys. Rev. A 20, 1608 (1979).

[62] G. Ruppeiner, "Thermodynamic Critical Fluctuation Theory?", Phys. Rev. Lett. 50, 287 (1983).

[63] G. Ruppeiner, "New thermodynamic fluctuation theory using path integrals", Phys. Rev. A $\mathbf{2 7}, 1116,1983$.

[64] G. Ruppeiner and C. Davis, Thermodynamic curvature of the multicomponent ideal gas, Phys. Rev. A 41, 2200, 1990.

[65] J. Shen, R. G. Cai, B. Wang, R. K. Su, "Thermodynamic Geometry and Critical Behavior of Black Holes", Int. J. Mod. Phys. A 22 (2007) 11-27, arXiv:gr-qc/0512035v1.

[66] J. E. Aman, I. Bengtsson, N. Pidokrajt, "Flat Information Geometries in Black Hole Thermodynamics", Gen. Rel. Grav. 38 (2006) 1305-1315, arXiv:gr-qc/0601119v1.

[67] J. E. Aman, I. Bengtsson, N. Pidokrajt, "Geometry of black hole thermodynamics", Gen. Rel. Grav. 35 (2003) 1733, arXiv:gr-qc/0304015v1.

[68] J. E. Aman, N. Pidokrajt, "Geometry of Higher-Dimensional Black Hole Thermodynamics", Phys. Rev. D 73 (2006) 024017, arXiv:hep-th/0510139v3.

[69] T. Sarkar, G. Sengupta, B. N. Tiwari, "On the Thermodynamic Geometry of BTZ Black Holes", JHEP 0611 (2006) 015, arXiv:hep-th/0606084v1.

[70] V. Balasubramanian, Jan de Boer, V. Jejjala, J. Simon, "The Library of Babel: On the origin of gravitational thermodynamics", JHEP 0512 (2005) 006, arXiv:hep-th/0508023v2.

[71] T. Sarkar, G. Sengupta, B. N. Tiwari, "Thermodynamic Geometry and Extremal Black Holes in String Theory", JHEP 0810, 076, 2008, arXiv:0806.3513v1 [hep-th].

[72] S. Bellucci, V. Chandra, B. N. Tiwari, "On the Thermodynamic Geometry of Hot QCD", to appear in Int. J. Mod. Phys. A, arXiv:0812.3792 v1 [hep-th].

[73] S. Bellucci, V. Chandra, B. N. Tiwari, "Thermodynamic Geometric Stability of Quarkonia states", arXiv:1010.4225v2 [hep-th].

[74] S. Bellucci, V. Chandra, B. N. Tiwari, "A geometric approach to correlations and quark number susceptibilities", arXiv:1010.4405 1 [hep-th].

[75] S. Bellucci, B. N. Tiwari, "Thermodynamic Geometry: Evolution, Correlation and Phase Transition", arXiv:1010.5148v2 [cond-mat.stat-mech].

[76] Landau, Lifshitz, "Statistical Mechanics", Pergaman Press (Hungary, 1980).

[77] D. Tranah and P. T. Landsberg, "Collective Phenomena", 3, 81 (1980). 
[78] G. Arcioni and E. L. Tellechea, "Stability and critical phenomena of black holes and black rings", Phys. Rev. D 72, 104021 (2005).

[79] J. E. Aman and N. Pidokrajt, "Geometry of higher-dimensional black hole thermodynamics", Phys. Rev. D 73, 024017 (2006).

[80] G. Ruppeiner, "Thermodynamic curvature and phase transitions in Kerr-Newman black holes", Phy. Rev. D 78, 024016 (2008)

[81] D. Bekenstein, "Information in the holographic universe", Sci. Am. 289, No. 2, 58-65 (2003).

[82] W. I. Taylor, "Adhering 0-branes to 6-branes and 8-branes", Nucl. Phys. B 508 (1997) 122, arXiv:hep-th/9705116

[83] R. R. Khuri and T. Ortín, "A Non-Supersymmetric Dyonic Extreme Reissner-Nordstrom Black Hole", Phys. Lett. B 373, 56 (1996), arXiv:hep-th/9512178.

[84] G. T. Horowitz, J. M. Maldacena, A. Strominger, "Nonextremal Black Hole Microstates and U-duality", Phys. Lett. B 383, 151 (1996), arXiv:hep-th/9603109.

[85] U. H. Danielsson, A. Guijosa, M. Kruczenski, "Brane-antibrane systems at finite temperature and the entropy of black branes", JHEP 0109, 011 (2001), arXiv:hep-th/0106201

[86] F. Larsen, "Rotating Kaluza-Klein Black Holes", Nucl. Phys. B 575 (2000) 211-230, arXiv:hep-th/9909102 v3.

[87] D. Rasheed, "The Rotating Dyonic Black Holes Of Kaluza-Klein Theory", Nucl. Phys. B 454 (1995) 379-401, arXiv:hep-th/9505038v1.

[88] F. Larsen, "Kaluza-Klein black holes in string theory", arXiv:hep-th/0002166.

[89] J. M. Maldacena, A. Strominger, "Statistical Entropy of Four-Dimensional Extremal Black Holes", Phys. Rev. Lett. 77, 428 (1996), arXiv:hep-th/9603060.

[90] R. Gopakumar, M. B. Green, "Instantons and Non-renormalisation in AdS/CFT", JHEP 9912015 (1999), arXiv:hep-th/9908020v2.

[91] J. Scherk, “Antigravity: A Crazy Idea?", Phys. Lett. B 88265 (1979).

[92] J. Scherk, in Supergravity, Proceedings of the 1979 Supergravity Workshop at Stony Brook, eds. P. van Nieuwenhuizen and D. Z. Freedman (NorthHolland, Amsterdam, 1979) p. 43.

[93] S. Bellucci and V. Faraoni, "The Equivalence principle, CP violations and the Higgs boson mass", Phys. Rev. D 49 (1994) 2922-2925.

[94] S. Bellucci, V. Faraoni, "Effects of the gravivector and graviscalar fields in N=2, N=8 supergravity", Phys. Lett. B 377 (1996) 55-59, arXiv:hep-ph/9605443

[95] J. B. Gutowski, W. Sabra, "General supersymmetric solutions of five-dimensional supergravity", JHEP 0510, 039 (2005), arXiv:hep-th/0505185

[96] M. Cvetic, M. J. Duff, P. Hoxha, J. T. Liu, H. Lu, J. X. Lu, R. Martinez-Acosta, C.N. Pope, H. Sati, T. A. Tran, "Embedding AdS black holes in ten-dimensions and eleven-dimensions", Nucl. Phys. B 558, 96 (1999), arXiv:hep-th/9903214

[97] M. Cvetic, G. W. Gibbons, H. Lu, C. N. Pope, "Rotating black holes in gauged supergravities; thermodynamics", supersymmetric limits, topological solitons and time machines", arXiv:hep-th/0504080

[98] A. Kostelecky, M. Perry, "Solitonic black holes in gauged N=2 supergravity", Phys. Lett. B 371, 191 (1996), arXiv:hep-th/9512222

[99] P. J. Silva, "Thermodynamics at the BPS bound for black holes in AdS", arXiv:hep-th/0607056

[100] D. Mateos, P. K. Townsend, "Supertubes", Phys. Rev. Lett. 87, 011602 (2001), arXiv:hep-th/0103030.

[101] B. C. Palmer, D. Marolf, "Counting supertubes", JHEP 0406, 028 (2004), arXiv:hep-th/0403025.

[102] D. Bak, Y. Hyakutake, N. Ohta, "Phase moduli space of supertubes", Nucl. Phys. B 696, 251 (2004), arXiv:hep-th/0404104

[103] D. Bak, Y. Hyakutake, S. Kim, N. Ohta, "A geometric look on the microstates of supertubes", Nucl. Phys. B 712, 115 (2005), arXiv:hep-th/0407253.

[104] A. Dabholkar, J. P. Gauntlett, J. A. Harvey, D. Waldram, "Strings as solitons and black holes as strings", Nucl. Phys. B 474, 85 (1996), arXiv:hep-th/9511053

[105] J. Cho, P. Oh, "Super D-helix", Phys. Rev. D 64, 106010 (2001), arXiv:hep-th/0105095. 
[106] D. Mateos, S. Ng, P.K. Townsend, "Supercurves", Phys. Lett. B 538, 366 (2002), arXiv:hep-th/0204062.

[107] J. C. Breckenridge, R. C. Myers, A. W. Peet, C. Vafa, "D-branes and spinning black holes", Phys. Lett. B 391, 93 (1997), arXiv:hep-th/9602065

[108] J. P. Gauntlett, R. C. Myers, P. K. Townsend, "Black holes of D = 5 supergravity", Class. Quant. Grav. 16, 1 (1999) arXiv:hep-th/9810204.

[109] A. Dabholkar, "Microstates of non-supersymmetric black holes", Phys. Lett. B 402, 53 (1997), arXiv:hep-th/9702050

[110] G. T. Horowitz, J. Polchinski, "A correspondence principle for black holes and strings", Phys. Rev. D 55, 6189 (1997), arXiv:hep-th/9612146

[111] R. Argurio, F. Englert, L. Houart, "Statistical entropy of the four-dimensional Schwarzschild black hole", Phys. Lett. B 426, 275 (1998), arXiv:hep-th/9801053.

[112] H. J. Sheinblatt, 'Statistical entropy of an extremal black hole with 0- and 6-brane charge', Phys. Rev. D 57, 2421 (1998), arXiv:hep-th/9705054

[113] A. Dhar, G. Mandal, "Probing 4-dimensional nonsupersymmetric black holes carrying D0- and D6brane charges", Nucl. Phys. B 531, 256 (1998), arXiv:hep-th/9803004

[114] A. Mikhailov, "Giant gravitons from holomorphic surfaces", JHEP 0011, 027 (2000), arXiv:hep-th/0010206.

[115] G. Mandal, N.V. Suryanarayana, "Counting 1/8-BPS dual giants", arXiv:hep-th/0606088.

[116] M. Awada, P. K. Townsend, "N=4 Maxwell-Einstein supergravity in five-dimensions and its SU(2) gauging", Nucl. Phys. B 255, 617 (1985).

[117] J. M. Maldacena, C. Nunez, "Supergravity description of field theories on curved manifolds and a no go theorem", Int. J. Mod. Phys. A 16, 822 (2001), arXiv:hep-th/0007018

[118] I. Bena, P. Kraus, "Microscopic description of black rings in AdS/CFT", JHEP 0412, 070 (2004), arXiv:hep-th/0408186

[119] I. Bena, P. Kraus, N. P. Warner, "Black rings in Taub-NUT", Phys. Rev. D72 (2005) 084019, arXiv:hep-th/0504142.

[120] M. Cyrier, M. Guica, D. Mateos, A. Strominger, "Microscopic Entropy of the Black Ring", Phys. Rev. Lett. 94, 191601 (2005), arXiv:hep-th/0411187

[121] O. Lunin, J. Maldacena, L. Maoz, "Gravity solutions for the D1-D5 system with angular momentum", arXiv:hep-th/0212210

[122] I. Bena, P. Kraus, "Microstates of the D1-D5-KK system", Phys. Rev. D 72 (2005) 025007, arXiv:hep-th/0503053

[123] S. Bellucci, B. N. Tiwari, "State-space Correlations and Stabilities", Phys. Rev. D 82 (2010) 084008, arXiv:0910.5309v1 [hep-th].

[124] S. Bellucci, B. N. Tiwari, "An Exact Fluctuating 1/2-BPS Configuration", JHEP 1005 (2010) 023, arXiv:0910.5314v2 [hep-th].

[125] S. Bellucci, B. N. Tiwari, "Black Strings, Black Rings and State-space Manifold", arXiv:1010.3832v1 [hep-th].

[126] S. Bellucci, B. N. Tiwari, "Thermodynamic Geometry and Hawking Radiation", JHEP 1011 (2010) 030, arXiv:1009.0633 [hep-th].

[127] H. Elvang, R. Emparan, D. Mateos, H. S. Reall, "A supersymmetric black ring", Phys. Rev. Lett. 93, 211302 (2004), arXiv:hep-th/0407065

[128] H. Elvang, R. Emparan, D. Mateos, H. S. Reall, "Supersymmetric 4D rotating black holes from 5D black rings", JHEP 08 (2005) 042, arXiv:hep-th/0504125

[129] R. Emparan, D. Mateos, P. K. Townsend, "Supergravity supertubes", JHEP 0107 (2001) 011, arXiv:hep-th/0106012

[130] I. Bena, "Splitting hairs of the three charge black hole", Phys. Rev. D 70, 105018, 2004, arXiv:hep-th/0404073 